\title{
Maps from Feigin and Odesskii's elliptic algebras to twisted homogeneous coordinate rings
}

\author{
Alex Chirvasitu ${ }^{1}$, Ryo Kanda ${ }^{2}$ and S. Paul Smith ${ }^{3}$ \\ ${ }^{1}$ Department of Mathematics, University at Buffalo, Buffalo, NY 14260-2900, USA; E-mail: achirvas@ buffalo.edu. \\ ${ }^{2}$ Department of Mathematics, Graduate School of Science, Osaka City University, 3-3-138, Sugimoto, Sumiyoshi, Osaka, \\ 558-8585, Japan; E-mail: ryo.kanda.math@gmail.com. \\ ${ }^{3}$ Department of Mathematics, Box 354350, University of Washington, Seattle, WA 98195, USA; \\ E-mail: smith@math.washington.edu.
}

Received: 26 June 2020; Revised: 11 November 2020; Accepted: 25 October 2020

2020 Mathematics Subject Classification: Primary - 14A22; Secondary - 16S38, 16W50, 14H52, 14F06

Key words and phrases: Elliptic algebra; Sklyanin algebra; twisted homogeneous coordinate ring; characteristic variety

\begin{abstract}
The elliptic algebras in the title are connected graded $\mathbb{C}$-algebras, denoted $Q_{n, k}(E, \tau)$, depending on a pair of relatively prime integers $n>k \geq 1$, an elliptic curve $E$ and a point $\tau \in E$. This paper examines a canonical homomorphism from $Q_{n, k}(E, \tau)$ to the twisted homogeneous coordinate ring $B\left(X_{n / k}, \sigma^{\prime}, \mathcal{L}_{n / k}^{\prime}\right)$ on the characteristic variety $X_{n / k}$ for $Q_{n, k}(E, \tau)$. When $X_{n / k}$ is isomorphic to $E^{g}$ or the symmetric power $S^{g} E$, we show that the homomorphism $Q_{n, k}(E, \tau) \rightarrow B\left(X_{n / k}, \sigma^{\prime}, \mathcal{L}_{n / k}^{\prime}\right)$ is surjective, the relations for $B\left(X_{n / k}, \sigma^{\prime}, \mathcal{L}_{n / k}^{\prime}\right)$ are generated in degrees $\leq 3$ and the noncommutative scheme $\operatorname{Proj}_{n c}\left(Q_{n, k}(E, \tau)\right)$ has a closed subvariety that is isomorphic to $E^{g}$ or $S^{g} E$, respectively. When $X_{n / k}=E^{g}$ and $\tau=0$, the results about $B\left(X_{n / k}, \sigma^{\prime}, \mathcal{L}_{n / k}^{\prime}\right)$ show that the morphism $\Phi_{\left|\mathcal{L}_{n / k}\right|}: E^{g} \rightarrow \mathbb{P}^{n-1}$ embeds $E^{g}$ as a projectively normal subvariety that is a scheme-theoretic intersection of quadric and cubic hypersurfaces.
\end{abstract}

\section{Contents}

1 Introduction $\quad \mathbf{2}$

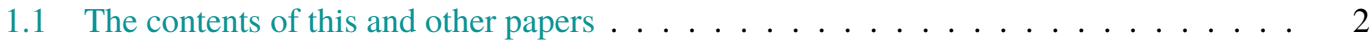

1.2 Some of what is known about $Q_{n, k}(E, \tau) \ldots \ldots \ldots \ldots \ldots$

1.3 The category $Q \operatorname{Gr}(A)$ when $A=Q_{n, k}(E, \tau) \ldots \ldots \ldots \ldots$

1.4 The definition of $Q_{n, k}(E, \tau) \ldots \ldots \ldots \ldots \ldots$

1.5 Review of results about $Q_{n, 1}(E, \tau) \ldots \ldots \ldots \ldots \ldots$

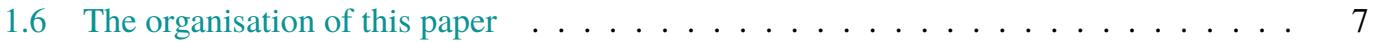

2 Twisted homogeneous coordinate rings $\quad \mathbf{8}$

2.1 Motivation: Projective normality and defining relations for abelian varieties . . . . . 8

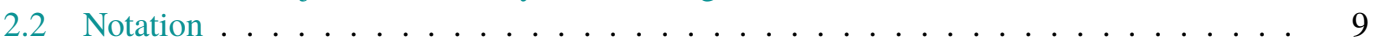

2.3 The twisted homogeneous coordinate rings $B(X, \sigma, \mathcal{L}) \ldots \ldots \ldots$

2.4 Using the $\operatorname{rings} B(X, \sigma, \mathcal{L}) \ldots \ldots \ldots \ldots \ldots \ldots \ldots \ldots$

2.5 Generators and relations for $B(X, \sigma, \mathcal{L}) \ldots \ldots \ldots \ldots \ldots$

2.6 Point modules for $B(X, \sigma, \mathcal{L}) \ldots \ldots \ldots \ldots \ldots$

(c) The Author(s), 2020. Published by Cambridge University Press. This is an Open Access article, distributed under the terms of the Creative Commons Attribution licence (http://creativecommons.org/licenses/by/4.0/), which permits unrestricted re-use, distribution, and reproduction in any medium, provided the original work is properly cited. 
3 The algebras $Q_{n, k}(E, \tau) \quad 17$

3.1 Some notation and results from $[\mathrm{CKS19b}] \ldots \ldots \ldots \ldots \ldots$

3.2 Twisted homogeneous coordinate rings related to $Q_{n, k}(E, \tau) \ldots \ldots$. . . . . . . . 19

4 Semistable and locally free $\mathcal{O}_{E}$-modules $\quad \mathbf{2 3}$

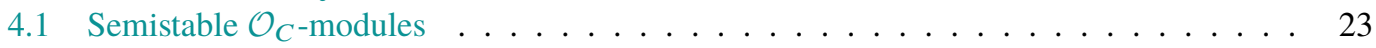

4.2 Surjectivity of multiplication maps . . . . . . . . . . . . . . . . . 24

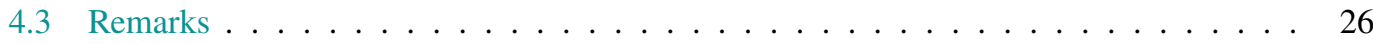

5 Twisted homogeneous coordinate rings of the form $B\left(S^{g} E, \sigma, \mathcal{L}\right) \quad 27$

5.1 Projective space bundles $\mathbb{P}(\mathcal{E})$ on an elliptic curve $E \ldots \ldots \ldots$. . . . . . . . 27

5.2 Multiplication of sections of invertible sheaves on $\mathbb{P}(\mathcal{E}) \ldots \ldots \ldots$

5.3 Symmetric powers of $E \ldots \ldots \ldots \ldots \ldots \ldots \ldots \ldots$

6 Relations for $B\left(S^{g} E, \sigma, \mathcal{L}\right) \quad 31$

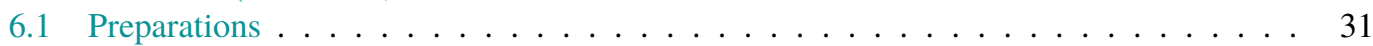

6.2 Surjectivity of multiplication maps . . . . . . . . . . . . . . . 32

7 The map $Q_{n, k}(E, \tau) \rightarrow B\left(S^{g} E, \sigma^{\prime}, \mathcal{L}_{n / k}^{\prime}\right)$ when the characteristic variety is $S^{g} E \quad 35$

7.1 Explicit description of $\sigma^{\prime}: X_{n / k} \rightarrow X_{n / k}$ when $X_{n / k} \cong S^{g} E \ldots \ldots \ldots$

7.2 The special case $Q_{5,2}(E, \tau) \ldots \ldots \ldots \ldots \ldots \ldots \ldots \ldots \ldots \ldots \ldots \ldots$

7.3 The map $\Psi_{n / k}: Q_{n, k}(E, \tau) \rightarrow B\left(S^{g} E, \sigma^{\prime}, \mathcal{L}_{n / k}^{\prime}\right) \ldots \ldots \ldots \ldots \ldots$

7.4 The algebras $Q_{2 k+1, k}(E, \tau) \ldots \ldots \ldots \ldots \ldots \ldots$

8 The rings $B\left(E^{g}, \sigma, \mathcal{L}_{n / k}\right) \quad 40$

8.1 The main result in this section . . . . . . . . . . . . . . . . . 40

8.2 Notation . . . . . . . . . . . . . . . . . . . . . 40

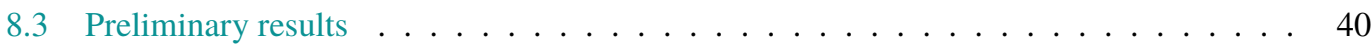

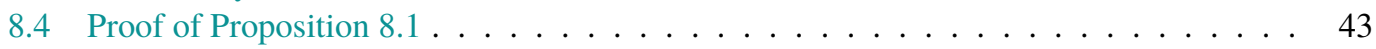

9 Relations for $B\left(E^{g}, \sigma, \mathcal{L}\right) \quad 44$

9.1 Notation . . . . . . . . . . . . . . . . . . . . . 44

9.2 The surjectivity and kernel of the map $Q_{n, k}(E, \tau) \rightarrow B\left(E^{g}, \sigma, \mathcal{L}_{n / k}\right)$ when $X_{n / k}=E^{g} \quad 45$ References

\section{Introduction}

For a fixed $n$ and $k$, the elliptic algebras $Q_{n, k}(E, \tau)$, defined by Feigin and Odesskii in 1989 [OF89], are noncommutative deformations of the polynomial ring on $n$ variables. Twisted homogeneous coordinate rings are noncommutative analogues (often deformations) of homogeneous coordinate rings (more precisely, section rings) for projective algebraic varieties. This paper uses the latter to study the former.

\subsection{The contents of this and other papers}

Always, $n$ and $k$ denote relatively prime integers $n>k \geq 1, E=\mathbb{C} / \Lambda$ is a complex elliptic curve and $\tau \in E$ is a (closed) point. We sometimes regard $\tau$ as a translation automorphism $\tau: E \rightarrow E$.

This is one of several papers we are writing about the algebras $Q_{n, k}(E, \tau)$. The first [CKS18] focused on their definition in terms of generators and relations, and established some immediate consequences of that definition. The second [CKS19b] examined its characteristic variety $X_{n / k}$, a projective algebraic variety that controls a large part of the structure and representation theory of $Q_{n, k}(E, \tau)$. Feigin and Odesskii identified a distinguished ample invertible sheaf $\mathcal{L}_{n / k}$ on $E^{g}$, the gth power of $E$, where $g$ is the 'length' of the negative continued fraction expression for $\frac{n}{k}$. This sheaf is generated by its global sections, the space of which has dimension $n$, so the complete linear system $\left|\mathcal{L}_{n / k}\right|$ provides a morphism $\Phi_{n / k}: E^{g} \rightarrow \mathbb{P}^{n-1}$, the image of which is $X_{n / k}$, by definition. The main result in [CKS19b] is that $X_{n / k}$ is isomorphic to the quotient $E^{g} / \Sigma_{n / k}$, where $\Sigma_{n / k}$ is a certain finite group; furthermore, $E^{g} / \Sigma_{n / k}$ is a bundle over a power of $E$ with fibres that are products of projective spaces. The third of 
our papers [CKS19a] examines the structure of $E^{g} / \Sigma_{n / k}$ in more detail: an étale cover of it that is a product of projective spaces and a power of $E$; a distinguished automorphism of it and its étale cover that is induced by a translation automorphism $\sigma: E^{g} \rightarrow E^{g}$ that 'controls' the noncommutativity of $Q_{n, k}(E, \tau)$. Another paper [CKS20] will show that $Q_{n, k}(E, \tau)$ has the same Hilbert series as the polynomial ring on $n$ variables when $\tau$ is not a torsion point of $E$.

This paper concerns homomorphisms from $Q_{n, k}(E, \tau)$ to noncommutative algebras $B(X, \sigma, \mathcal{L})$ defined in terms of a scheme $X$, an automorphism $\sigma: X \rightarrow X$ and an invertible $\mathcal{O}_{X}$-module $\mathcal{L}$. The algebras $B(X, \sigma, \mathcal{L})$ are the 'twisted homogeneous coordinate rings' in the title. They are noncommutative analogues of the section rings $\oplus_{i \geq 0} H^{0}\left(X, \mathcal{L}^{\otimes i}\right)$.

Our main result is as follows (some of the notation is explained later in this introduction):

\section{Theorem 1.1.}

(1) There are nontrivial graded $\mathbb{C}$-algebra homomorphisms

$$
Q_{n, k}(E, \tau) \stackrel{\Psi_{n / k}}{\longrightarrow} B\left(X_{n / k}, \sigma^{\prime}, \mathcal{L}_{n / k}^{\prime}\right) \longrightarrow B\left(E^{g}, \sigma, \mathcal{L}_{n / k}\right)^{\Sigma_{n / k}} \subseteq B\left(E^{g}, \sigma, \mathcal{L}_{n / k}\right) .
$$

(2) The quotient categories $\mathrm{Q} \operatorname{Gr}\left(B\left(X_{n / k}, \sigma^{\prime}, \mathcal{L}_{n / k}^{\prime}\right)\right)$ and $\mathrm{QGr}\left(B\left(E^{g}, \sigma, \mathcal{L}_{n / k}\right)\right)$ are equivalent to the categories $\mathrm{Qcoh}\left(X_{n / k}\right)$ and $\mathrm{Qcoh}\left(E^{g}\right)$, respectively.

If all the integers $n_{1}, \ldots, n_{g}$ in the negative continued fraction for $\frac{n}{k}$ are $\geq 3$ (resp., exactly one of $n_{1}$ and $n_{g}$ is $\geq 3$ and the other $n_{i}$ are 2), then

(3) $X_{n / k}$ is isomorphic to the gth power $E^{g}$ (resp., the gth symmetric power $S^{g} E$ );

(4) the homomorphism $Q_{n, k}(E, \tau) \rightarrow B\left(X_{n / k}, \sigma^{\prime}, \mathcal{L}_{n / k}^{\prime}\right)$ is surjective; equivalently, $B\left(X_{n / k}, \sigma^{\prime}, \mathcal{L}_{n / k}^{\prime}\right)$ is generated by elements of degree one;

(5) the relations for $B\left(X_{n / k}, \sigma^{\prime}, \mathcal{L}_{n / k}^{\prime}\right)$ are generated in degrees $\leq 3$;

(6) $X_{n / k}$ is a closed subvariety of the noncommutative scheme $\operatorname{Proj}_{n c}\left(Q_{n, k}(E, \tau)\right)$ - that is, there are functors

$$
i_{*}, i^{!}: \operatorname{QGr}\left(Q_{n, k}(E, \tau)\right) \longrightarrow \operatorname{QGr}\left(B\left(X_{n / k}, \sigma^{\prime}, \mathcal{L}_{n / k}^{\prime}\right)\right)
$$

and

$$
i^{*}: \operatorname{QGr}\left(B\left(X_{n / k}, \sigma^{\prime}, \mathcal{L}_{n / k}^{\prime}\right)\right) \longrightarrow \operatorname{QGr}\left(Q_{n, k}(E, \tau)\right)
$$

forming an adjoint triple $i^{*} \dashv i_{*} \dashv i^{!}$, and $i_{*}$ is a fully faithful functor whose essential image is closed under subquotients.

Proof.

(1) Corollary 3.6 and Theorem 3.2(5).

(2) Theorem 2.4, Corollary 2.7, Proposition 3.1 and Theorem 3.2.

(3) $\$ 3.1 .7$.

(4),(5) Theorem 7.5, Proposition 8.1 and Theorem 9.7.

(6) $\$ 1.3 .1$.

The perspective of noncommutative algebraic geometry is illuminating. The algebra $Q_{n, k}(E, \tau)$ is a homogeneous coordinate ring for a noncommutative analogue $\operatorname{Proj}_{n c}\left(Q_{n, k}(E, \tau)\right)$ of the projective space $\mathbb{P}^{n-1}$. The homomorphism $Q_{n, k}(E, \tau) \rightarrow B\left(X_{n / k}, \sigma^{\prime}, \mathcal{L}_{n / k}^{\prime}\right)$ induces a 'map' $X_{n / k} \rightarrow$ $\operatorname{Proj}_{n c}\left(Q_{n, k}(E, \tau)\right)$. When $X_{n / k}$ is $E^{g}$ or $S^{g} E$, this map is a 'closed immersion' - that is, there are noncommutative analogues of the usual inverse and direct image functors that allow one to carry information from $Q \operatorname{coh}\left(X_{n / k}\right)$ to an analogous category of graded $Q_{n, k}(E, \tau)$-modules.

When $\tau=0$, Theorem 1.1(5) shows that the image of $E^{g}$ in $\mathbb{P}^{n-1}$ under $\Phi_{n / k}$ is a scheme-theoretic intersection of quadric and cubic hypersurfaces (we do not know if this follows from known results). Thus, in a sense, the situation for $\tau \neq 0$ is exactly the same. That result also recovers the less well-known 
fact that the image of $\Phi_{|\mathcal{L}|}: S^{g} E \rightarrow \mathbb{P}^{(m-1) g}$, where the class of $\mathcal{L}$ in the Néron-Severi group of $S^{g} E$ is $D+(m-1) F$ with $m \geq 3$ (see $\$ 5.1 .3$ for notation), is a scheme-theoretic intersection of quadric and cubic hypersurfaces. Again, we note that the noncommutative case is perfectly analogous to the classical case.

\subsection{Some of what is known about $Q_{n, k}(E, \tau)$}

The algebras $Q_{n, k}(E, 0)$ and $Q_{n, n-1}(E, \tau)$ are polynomial rings on $n$ variables ([CKS18, Propositions 5.1 and 5.5]). The algebras $Q_{n, 1}(E, \tau)$ are commonly called Sklyanin algebras.

For a fixed $E$ and $n$, Odesskii and Feigin showed that the algebras $Q_{n, 1}(E, \tau)$ provide a flat family of deformations of the polynomial ring on $n$ variables for all $\tau$ in a countable intersection of Zariski-open neighbourhoods of 0 . Tate and Van den Bergh made a careful analysis of the algebras $Q_{n, 1}(E, \tau)$ for all $\tau$ and all elliptic curves defined over an arbitrary field [TVdB96]. Among other things, they showed that as $E$ and $\tau$ vary, the algebras $Q_{n, 1}(E, \tau)$ form a flat family of deformations of the polynomial ring on $n$ variables - that is, for all $\tau$, the dimensions of the homogeneous components of $Q_{n, 1}(E, \tau)$ are the same as those of the polynomial ring on $n$ variables.

This paper concerns $Q_{n, k}(E, \tau)$ when $k>1$ and $\tau$ is arbitrary.

Tate and Van den Bergh showed that $Q_{n, 1}(E, \tau)$ has the following properties:

(1) It is a connected graded left and right Noetherian algebra having the same Hilbert series as the polynomial ring on $n$ variables (with its standard grading).

(2) It has no zero divisors.

(3) It is a Koszul algebra.

(4) It is a finite module over its centre if and only if $\tau$ has finite order. ${ }^{1}$

(5) It is Cohen-Macaulay.

(6) It has the Auslander property.

(7) It is an Artin-Schelter regular algebra [AS87].

Definitions of the last three properties can be found in [Lev92]. We expect that every $Q_{n, k}(E, \tau)$ has these properties. In [CKS20], we show that $Q_{n, k}(E, \tau)$ has the same Hilbert series as the polynomial ring on $n$ variables and is a Koszul algebra, provided that $\tau$ is not a torsion point.

\subsection{The category $Q \operatorname{Gr}(A)$ when $A=Q_{n, k}(E, \tau)$}

Let $\mathbb{k}$ be a field and $A$ a finitely generated connected graded $\mathbb{k}$-algebra. Let $\operatorname{Gr}(A)$ denote the category of $\mathbb{Z}$-graded left $A$-modules. We write $\operatorname{Fdim}(A)$ for the full subcategory of $\operatorname{Gr}(A)$ consisting of those modules that are the sum of their finite-dimensional submodules and define the quotient category

$$
\operatorname{QGr}(A):=\frac{\operatorname{Gr}(A)}{\operatorname{Fdim}(A)} \text {. }
$$

If $A$ is a finitely generated commutative connected $\mathbb{k}$-algebra generated by its degree-one component, then $\mathrm{Q} \operatorname{Gr}(A)$ is equivalent to the category of quasi-coherent sheaves on the projective scheme $\operatorname{Proj}(A)$. Even when $A$ is not commutative, the category $\mathrm{QGr}(A)$ often behaves like the category of quasi-coherent sheaves on a projective scheme.

\subsection{1.}

Suppose $\mathcal{L}_{n / k}$ is very ample, or equivalently, all integers in the 'negative' continued fraction for $\frac{n}{k}$ are $\geq 3$ (see §3.1.3), or equivalently, the natural map $E^{g} \rightarrow X_{n / k}$ is an isomorphism. Then $B\left(X_{n / k}, \sigma^{\prime}, \mathcal{L}_{n / k}^{\prime}\right)=$

\footnotetext{
${ }^{1}$ Tate and Van den Bergh proved that $Q_{n, k}(E, \tau)$ is finite over its centre if $\tau$ has finite order. The converse follows from Corollary 3.7 and Theorem 1.1(4).
} 
$B\left(E^{g}, \sigma, \mathcal{L}_{n / k}\right)$ and the homomorphism

$$
\Psi_{n / k}: Q_{n, k}(E, \tau) \longrightarrow B\left(E^{g}, \sigma, \mathcal{L}_{n / k}\right)
$$

in Theorem 1.1 is surjective, or equivalently, $B\left(E^{g}, \sigma, \mathcal{L}_{n / k}\right)$ is generated by elements of degree one (§8.1). The sheaf $\mathcal{L}_{n / k}$ is $\sigma$-ample (see $\$ 2.3 .2$ and Theorem 3.2(6)), so a result of Artin and Van den Bergh [AVdB90] (see §2.3.3) tells us that $\mathrm{QGr}\left(B\left(E^{g}, \sigma, \mathcal{L}_{n / k}\right)\right)$ is equivalent to $\mathrm{Qcoh}\left(E^{g}\right)$. Combining this with the main result in [Smi04] (see [Smi16, Theorem 1.2]) implies that there are functors

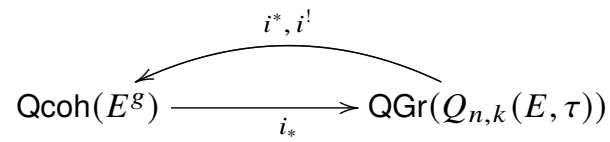

satisfying the properties in Theorem 1.1(6). The claim for the cases $\frac{n}{k}=[m, 2, \ldots, 2]$ and $[2, \ldots, 2, m]$ $(m \geq 3)$ follows from a similar argument using Theorem 5.7.

\subsection{The definition of $Q_{n, k}(E, \tau)$}

Fix a point $\eta \in \mathbb{C}$ lying in the upper half-plane. Let $\Lambda=\mathbb{Z}+\mathbb{Z} \eta$ and define $E=\mathbb{C} / \Lambda$. Let $\Theta_{n}(\Lambda)$ be the space of theta functions defined in [CKS18, §2.1], and let $\theta_{0}(z), \ldots, \theta_{n-1}(z)$ be the basis for $\Theta_{n}(\Lambda)$ defined in [CKS18, Proposition 2.6]. For all $\tau \in \mathbb{C}-\frac{1}{n} \Lambda$, we define $Q_{n, k}(E, \tau)$ to be the free algebra $\mathbb{C}\left\langle x_{0}, \ldots, x_{n-1}\right\rangle$ modulo the $n^{2}$ relations

$$
\sum_{r \in \mathbb{Z}_{n}} \frac{\theta_{j-i+r(k-1)}(0)}{\theta_{j-i-r}(-\tau) \theta_{k r}(\tau)} x_{j-r} x_{i+r}=0, \quad(i, j) \in \mathbb{Z}_{n}^{2}
$$

For the rest of this introduction, we assume $\tau \notin \frac{1}{n} \Lambda$. This ensures that the denominators in equation (1.3) are nonzero. In [CKS18, Definition 3.11], we defined $Q_{n, k}(E, \tau)$ for all $\tau \in E$.

In [CKS20] it is shown that the $n^{2}$ relations in equation (1.3) span an $\left(\begin{array}{l}n \\ 2\end{array}\right)$-dimensional space when $\tau$ is not a torsion point.

By [CKS18, Proposition 3.22], $Q_{n, k}(E, \tau) \cong Q_{n, k}(E,-\tau)=Q_{n, k}(E, \tau)^{\mathrm{op}}$.

Although the relations for $Q_{n . k}(E, \tau)$ seem to have no meaning at first sight, there are two perspectives that make them less mysterious. One involves $R$-matrices and the other involves an identity for theta functions on $g$ variables.

\subsection{1.}

The relations in equation (1.3) come from Belavin's elliptic solutions to the quantum Yang-Baxter equation. Let $V$ be a $\mathbb{C}$-vector space with basis $e_{0}, \ldots, e_{n-1}$. For each $z \in \mathbb{C}$, let $R(z): V \otimes V \rightarrow V \otimes V$ be the linear operator

$$
R(z)\left(e_{i} \otimes e_{j}\right):=\sum_{r \in \mathbb{Z}_{n}} \frac{\theta_{j-i+r(k-1)}(-z+\tau)}{\theta_{k r}(\tau) \theta_{j-i-r}(-z)} e_{j-r} \otimes e_{i+r} .
$$

As conjectured by Belavin [Be180] and later proved by Cherednik [Che82], Chudnovsky and Chudnovsky [CC81] and Tracy [Tra85], when $k=1$ these operators satisfy the equation

$$
R(u)_{12} R(u+v)_{23} R(v)_{12}=R(v)_{23} R(u+v)_{12} R(u)_{23} .
$$

Clearly,

$$
Q_{n, k}(E, \tau)=\frac{T V}{(\operatorname{im} R(\tau))},
$$


the right-hand side of which denotes the quotient of the tensor algebra on $V$ by the ideal generated by the image of $R(\tau)$. In [CKS20], we use the fact that $R(z)$ satisfies the quantum Yang-Baxter equation to show that $Q_{n, k}(E, \tau)$ has the same Hilbert series as the polynomial ring on $n$ variables when $\tau$ is not a torsion point.

\subsection{2.}

The second 'explanation' for the relations involves an $n$-dimensional space $\Theta_{n / k}(\Lambda)$ of theta functions in $g$ variables, where $g$ is the number in $\$ 1.3 .1$. The Heisenberg group $H_{n}$ of order $n^{3}$ acts in a natural way on $\Theta_{n / k}(\Lambda)$, and there is a basis $w_{0}, \ldots, w_{n-1}$ for $\Theta_{n / k}(\Lambda)$ that transforms in a nice way with respect to the 'standard' generators for $H_{n}$ (see [CKS19b, §5.1.1]). There is an identity

$$
\sum_{r \in \mathbb{Z}_{n}} \frac{\theta_{\beta-\alpha+r(k-1)}(0)}{\theta_{\beta-\alpha-r}(-\tau) \theta_{r k}(\tau)} w_{\beta-r}(\mathrm{z}) w_{\alpha+r}(\sigma(\mathrm{z}))=0
$$

in which $z \in \mathbb{C}^{g}$ and $\sigma$ is a certain automorphism of $\mathbb{C}^{g}$ defined in $\S 3.1 .4$. Compare equations (1.4) and (1.3): if one identifies $x_{\alpha}$ with $w_{\alpha}$, then equation (1.4) tells us that the relations for $Q_{n, k}(E, \tau)$ vanish on the graph of $\sigma$.

\subsubsection{The relations for $Q_{2 k+1, k}(E, \tau)$}

This case, which includes the 3-dimensional Sklyanin algebra $Q_{3,1}(E, \tau)$, is special. Since $\frac{2 k+1}{k}=$ $[3,2, \ldots, 2]$, where there are $k-1$ twos, $X_{n / k} \cong S^{k} E$. The automorphism $\sigma^{\prime}: S^{k} E \rightarrow S^{k} E$ is $\left(\left(z_{1}, \ldots, z_{k}\right)\right) \mapsto\left(\left(z_{1}+\tau, \ldots, z_{k}+\tau\right)\right)$ (Proposition 7.2). The degree-one component, say $V$, of $Q_{2 k+1, k}(E, \tau)$ can be viewed as linear forms on $\mathbb{P}^{n-1}=\mathbb{P}^{2 k}$, so $V^{\otimes 2}$ can be viewed as bilinear forms on $\mathbb{P}^{2 k} \times \mathbb{P}^{2 k}$.

Theorem 1.2 (Proposition 7.8). If $\tau \in E$ is not a 2-torsion point, then the quadratic relations for $Q_{2 k+1, k}(E, \tau)$ are exactly those elements of $V^{\otimes 2}$ that vanish on the graph of the automorphism $\sigma^{\prime}$ : $S^{k} E \rightarrow S^{k} E$.

\subsection{Review of results about $Q_{n, 1}(E, \tau)$}

\subsection{1.}

The algebras $Q_{3,1}(E, \tau)$ first appeared in Artin and Schelter's classification of 3-dimensional regular algebras [AS87]. There, the algebras $Q_{3,1}(E, \tau)$ belonged to a slightly larger class of algebras $A_{a, b . c}$ parametrised by points $(a, b, c) \in \mathbb{P}^{2}$ and defined as $\mathbb{C}\left\langle x_{0}, x_{1}, x_{2}\right\rangle /\left(r_{0}, r_{1}, r_{2}\right)$, where $r_{i}=a x_{i} x_{i+1}+$ $b x_{i+1} x_{i}+c x_{i+2}^{2}$ (see [AS87, (10.36) and 10.37(i)], and the remark at [ATVdB90, p. 38] to the effect that the conjecture in $[A S 87,10.37(\mathrm{i})]$ is true). Artin, Tate and Van den Bergh showed that $A_{(a, b, c)}$ is a 3-dimensional regular algebra if and only if $(a, b, c) \in \mathbb{P}^{2}-\{12$ points $\}$. To do that, they introduced the notion of a twisted homogeneous coordinate ring [ATVdB90] (Odesskii and Feigin discovered this notion around the same time [FO89, p. 7] and [OF89, p. 208]), and showed that there is a surjective homomorphism

$$
\Psi: Q_{3,1}(E, \tau) \longrightarrow B\left(E, \tau, \mathcal{L}_{3}\right)
$$

where $\mathcal{L}_{3}$ is an invertible $\mathcal{O}_{E}$-module of degree 3 and $\operatorname{ker}(\Psi)$ is generated by a degree-three central element, say $\Omega$. $^{2}$ They exploit this, and the fact that $\operatorname{QGr}\left(B\left(E, \tau, \mathcal{L}_{3}\right)\right)$ is equivalent to $Q \operatorname{coh}(E)$, to show that $Q_{3,1}(E, \tau)$ has properties (1)-(7) in $\S 1.2$. One should think of $B\left(X, \tau, \mathcal{L}_{3}\right)$ as a homogeneous coordinate ring of $E$, albeit a noncommutative, or twisted, one. In a similar spirit, one should view $Q_{3,1}(E, \tau)$ as a noncommutative algebra that behaves as if it is the homogeneous coordinate ring of a

\footnotetext{
${ }^{2}$ When $\tau=0$, the vanishing locus of $\Omega$ is the curve $a b c\left(x^{3}+y^{3}+z^{3}\right)=\left(a^{3}+b^{3}+c^{3}\right) x y z-$ which is nonsingular if and only if $a b c \neq 0$ and $(3 a b c)^{3} \neq\left(a^{3}+b^{3}+c^{3}\right)^{3}$ - and $\Psi$ is the familiar map from the polynomial ring on 3 variables to the homogeneous coordinate ring of the image of $E$ under the morphism $E \rightarrow \mathbb{P}\left(H^{0}\left(E, \mathcal{L}_{3}\right)^{*}\right)$.
} 
noncommutative analogue of the projective plane $\mathbb{P}^{2}$. The element $\Omega$ plays the role of a cubic equation whose zero locus is $E$.

\subsection{2.}

Similar results hold for $Q_{4,1}(E, \tau)$. Sklyanin used Baxter's elliptic solution to the quantum Yang-Baxter equation to define a family of algebras $A_{4}(E, \tau)$ [Sk182, Sk183]. As mentioned in [ST94, p. 20], there is an isomorphism

$$
Q_{4,1}(E, \tau) \cong A_{4}(E, 2 \tau) .
$$

Smith and Stafford [SS92, §3] showed that there is a surjective homomorphism $A_{4}(E, \tau) \rightarrow B\left(E, \tau, \mathcal{L}_{4}\right)$, and hence a surjective homomorphism

$$
\Psi: Q_{4,1}(E, \tau) \longrightarrow B\left(E, 2 \tau, \mathcal{L}_{4}\right),
$$

where $\mathcal{L}_{4}$ is an invertible $\mathcal{O}_{E}$-module of degree 4 , and showed that the kernel of $\Psi$ is generated by a regular sequence consisting of two degree-two central elements, say $\Omega_{1}$ and $\Omega_{2}$ ([SS92, Corollary 3.9 and Theorem 5.4]). They used this and the fact that $\mathrm{Q} \operatorname{Gr}\left(B\left(E, \tau, \mathcal{L}_{4}\right)\right)$ is equivalent to $\mathrm{Qcoh}(E)$ to show that $Q_{4,1}(E, \tau)$ has properties (1)-(7) in $\S 1.2$. One thinks of $Q_{4,1}(E, \tau)$ as if it is the homogeneous coordinate ring of a noncommutative analogue $\operatorname{Proj}_{n c}\left(Q_{4,1}(E, \tau)\right)$ of the projective space $\mathbb{P}^{3}$, and of $\Omega_{1}$ and $\Omega_{2}$ as if they are the defining equations of $E$ presented as the intersection of two 'noncommutative quadrics' in $\operatorname{Proj}_{n c}\left(Q_{4,1}(E, \tau)\right)$. This theme is elaborated on in [SVdB13].

\subsection{3.}

As stated immediately after the proof of [FO89, Theorem 3.1], for all $n \geq 3$ there is a surjective homomorphism

$$
\Psi: Q_{n, 1}(E, \tau) \longrightarrow B\left(E,(n-2) \tau, \mathcal{L}_{n}\right)
$$

with $\mathcal{L}_{n}$ an invertible $\mathcal{O}_{E}$-module of degree $n$ (see [TVdB96, §4.1]). All degree- $n$ invertible $\mathcal{O}_{E}$ modules are pullbacks of each other along suitable translation automorphisms, so the isomorphism class of $B\left(E,(n-2) \tau, \mathcal{L}_{n}\right)$ does not depend on the choice of $\mathcal{L}_{n}$. When $n \geq 5$ it is difficult to use the surjectivity of $\Psi$ to obtain information about $Q_{n, 1}(E, \tau)$, because $\operatorname{ker}(\Psi)$ is no longer generated by a regular sequence of central elements; this is analogous to the fact that the image of $E$ under the morphism $\Phi_{\left|\mathcal{L}_{n}\right|}: E \subseteq \mathbb{P}\left(H^{0}\left(E, \mathcal{L}_{n}\right)^{*}\right)$ is a complete intersection if and only if $n=3,4 .{ }^{3}$

\subsection{The organisation of this paper}

Section 2 concerns twisted homogeneous coordinate rings. It records important results due to Artin and Van den Bergh and to Keeler, and a few results that are not in the literature (but should be). Some of those are surely known to others. Corollaries 2.6 and 2.7, which appear to be new, give a criterion for $\sigma$-ampleness that is particularly useful for the types of twisted homogeneous coordinate rings that appear in the study of $Q_{n, k}(E, \tau)$.

$\$ 3.1$ records some results and notation from our earlier papers about $Q_{n, k}(E, \tau)$ that are used in this paper. We discuss maps from $Q_{n, k}(E, \tau)$ to twisted homogeneous coordinate rings in \$3.2. The main results there are Theorem 3.2 and Corollary 3.6. We also want to emphasise the isomorphism $B\left(X_{n / k}, \sigma^{\prime}, \mathcal{L}_{n / k}^{\prime}\right) \cong B\left(X_{n / k},\left(\sigma^{\prime}\right)^{-1}, \mathcal{L}_{n / k}^{\prime}\right)$ in Theorem 3.4. This and the anti-isomorphism $B\left(X, \sigma^{-1}, \mathcal{L}\right)^{\mathrm{op}} \cong B(X, \sigma, \mathcal{L})$ in Proposition 2.2 allow us to reconcile some sign differences that arise

\footnotetext{
${ }^{3}$ This is well known. The case $n=3$ is trivial. When $n=4, E$ is an intersection of two quadrics (see, e.g., [Har77, Exercise IV.3.6] or [Hul86, Chapter III]. Since the degree- $n$ elliptic normal curve $E \subseteq \mathbb{P}^{n-1}$ is not contained in any hyperplane, if it is a complete intersection it would be a complete intersection of $n-2$ hypersurfaces of degree $\geq 2$, so it would have degree $\geq 2^{n-2}$; however, if $n>4$, then $n<2^{n-2}$.
} 
in comparing various papers. These (anti-)isomorphisms and the homomorphisms in $\$ 3.2$ are compatible with the observation in [CKS18, Proposition 3.22] that $Q_{n, k}(E, \tau) \cong Q_{n, k}(E,-\tau)=Q_{n, k}(E, \tau)^{\mathrm{op}}$.

Our questions about the degrees of minimal sets of generators and relations for $B\left(X_{n / k}, \sigma^{\prime}, \mathcal{L}_{n / k}^{\prime}\right)$ often reduce to this: if $\mathcal{F}$ and $\mathcal{G}$ are locally free ${ }^{4} \mathcal{O}_{X}$-modules, when is the natural map

$$
H^{0}(X, \mathcal{F}) \otimes H^{0}(X, \mathcal{G}) \rightarrow H^{0}(X, \mathcal{F} \otimes \mathcal{G})
$$

surjective? This question is of broad interest in algebraic geometry and has been studied a great deal. We prove several new results of this form in the later sections of the paper. Most of those results are for varieties $X$ for which there is a surjective morphism $\pi: X \rightarrow E$. The proofs often reduce to the question of whether $H^{0}\left(E, \pi_{*} \mathcal{F}\right) \otimes H^{0}\left(E, \pi_{*} \mathcal{G}\right) \rightarrow H^{0}\left(E, \pi_{*} \mathcal{F} \otimes \pi_{*} \mathcal{G}\right)$ is surjective. It usually turns out in the cases of interest to us that $\pi_{*} \mathcal{F}$ and $\pi_{*} \mathcal{G}$ are semistable locally free $\mathcal{O}_{E}$-modules. For this reason, section 4 collects a number of standard results about semistable $\mathcal{O}_{E}$-modules. We also prove the following result that we found particularly useful:

Theorem 1.3 (Theorem 4.9). Let $\mathcal{U}$ and $\mathcal{V}$ be semistable locally free coherent $\mathcal{O}_{E}$-modules of slopes $\mu(\mathcal{U})$ and $\mu(\mathcal{V})$. If $\mathcal{U}$ and $\mathcal{V}$ are generated by their global sections and

$$
\frac{1}{\mu(\mathcal{U})}+\frac{1}{\mu(\mathcal{V})}<1,
$$

then the multiplication map $H^{0}(E, \mathcal{U}) \otimes H^{0}(E, \mathcal{V}) \rightarrow H^{0}(E, \mathcal{U} \otimes \mathcal{V})$ is surjective.

Although our ultimate interest is the specific twisted homogeneous coordinate rings $B\left(X_{n / k}, \sigma^{\prime}, \mathcal{L}_{n / k}^{\prime}\right)$, we often prove results in greater generality. For example, Proposition 5.6 provides a result about the surjectivity of the map in (1.5) when $X$ is a projective space bundle over $E$. In section 6 we show that $B\left(S^{g} E, \sigma, \mathcal{L}\right)$ is generated in degree one and has relations of degrees 2 and 3 for all $g \geq 2$, all translation automorphisms $\sigma: S^{g} E \rightarrow S^{g} E$ and all invertible $\mathcal{L}$ whose class in the Néron-Severi group is $a D+b F$ with $a \geq 1$ and $b \geq 2 .{ }^{5}$ Likewise, section 9 shows that the relations for $B\left(E^{g}, \sigma, \mathcal{L}_{n / k}\right)$ are generated in degree $\leq 3$ for all translation automorphisms $\sigma: E^{g} \rightarrow E^{g}$ when $\mathcal{L}_{n / k}$ is very ample.

\section{Twisted homogeneous coordinate rings}

In this section we mostly work over an algebraically closed field $\mathbb{k}$. Always, $E$ denotes an elliptic curve defined over $\mathbb{k}$ and $\mathbb{P}^{n}$ denotes the projective space $\mathbb{P}_{\mathbb{k}}^{n}$.

We always assume $\mathbb{K}=\mathbb{C}$ when we discuss $Q_{n, k}(E, \tau)$, because its definition involves theta functions.

\subsection{Motivation: Projective normality and defining relations for abelian varieties}

Nothing in this section is used later in the paper. Its purpose is to explain how the results about $B\left(E^{g}, \sigma, \mathcal{L}_{n / k}\right.$ ) (when all the $n_{i}$ in the continued fraction for $\frac{n}{k}$ are $\geq 3$ ) in parts (4) and (5) of Theorem 1.1 fit into the theme of defining relations for abelian varieties: when $\sigma=\mathrm{id}, B\left(E^{g}, \sigma, \mathcal{L}_{n / k}\right)$ is the section ring $S\left(E^{g}, \mathcal{L}_{n / k}\right)$, so those results say that $\mathcal{L}_{n / k}$ is normally generated and the image of $E^{g}$ under the embedding $\Phi_{\left|\mathcal{L}_{n / k}\right|}: E^{g} \rightarrow \mathbb{P}^{n-1}$ is a scheme-theoretic intersection of quadrics and cubics.

Let $X$ be a projective algebraic variety, $\mathcal{L}$ a very ample invertible $\mathcal{O}_{X}$-module and $\Phi_{|\mathcal{L}|}: X \rightarrow \mathbb{P}^{r}=$ $\mathbb{P}\left(H^{0}(X, \mathcal{L})^{*}\right)$ the associated embedding. We identify $X$ with its image in $\mathbb{P}^{r}$, denote by $I_{X}$ the largest graded ideal in $\mathbb{k}\left[x_{0}, \ldots, x_{r}\right]$ vanishing on $X$ and write $S(X)$ for the homogeneous coordinate ring $\mathbb{k}\left[x_{0}, \ldots, x_{r}\right] / I_{X}$. The following statements are equivalent:

\footnotetext{
${ }^{4}$ All our locally free sheaves are coherent.

${ }^{5}$ Given the basis $\{D, F\}$ for NS $\left(S^{g} E\right)$ in $\$ 5.1 .3$, if $[\mathcal{L}]=a D+b F$ with $a \geq 1$ and $b \geq 2$, then $\mathcal{L}$ is ample and generated by its global sections.
} 
(1) The restriction map

$$
\mathbb{k}\left[x_{0}, \ldots, x_{r}\right]=\bigoplus_{k=0}^{\infty} H^{0}\left(\mathbb{P}^{r}, \mathcal{O}_{\mathbb{P}^{r}}(k)\right) \longrightarrow S(X, \mathcal{L}):=\bigoplus_{k=0}^{\infty} H^{0}\left(X, \mathcal{L}^{\otimes k}\right)
$$

is surjective.

(2) $S(X)$ is integrally closed.

(3) The map $S(X) \rightarrow S(X, \mathcal{L})$ is an isomorphism.

(4) $S(X, \mathcal{L})$ is generated by its degree-one component.

If one - hence each - of these conditions holds, we say that $\mathcal{L}$ is normally generated and that the subvariety $X \subseteq \mathbb{P}^{r}$ is projectively normal. A fundamental problem in algebraic geometry is to decide when this happens and, when it does, to determine the degrees of a minimal set of relations for $X \subseteq \mathbb{P}^{r}$.

Let $X$ be a complex abelian variety. The theorem in the introduction to [PP04] provides a short history of what is known about $S(X, \mathcal{L})$. Those results have the following flavor: if $\mathcal{L}$ is a sufficiently high power of an ample invertible sheaf, perhaps with an additional hypothesis about its base locus or global generation, then $S(X, \mathcal{L})$ is generated by its degree-one component and the kernel of the map $S(X) \rightarrow S(X, \mathcal{L})$ is generated by elements of degree two, and perhaps degree three. Most of those results are subsumed by [PP04, Thm. 6.1]: if $\mathcal{M}$ is an ample invertible sheaf on an abelian variety $X$, then $\mathcal{M}^{\otimes 3}$ is very ample (Lefschetz's theorem [Kem91, Theorem 2.11]) and is normally generated (Koizumi's theorem [Koi76, Corollary 4.7]), and the kernel of the map $S(X) \rightarrow S\left(X, \mathcal{M}^{\otimes 3}\right)$ is generated by elements of degrees 2 and 3 (Mumford's theorem [PP04, Theorem (7), p. 168]).

The twisted homogeneous coordinate rings $B(X, \sigma, \mathcal{L})$ defined in $\$ 2.3$ are noncommutative analogues of $S(X, \mathcal{L})\left(B\left(X, \mathrm{id}_{X}, \mathcal{L}\right)=S(X, \mathcal{L})\right)$, and the same questions about $B(X, \sigma, \mathcal{L})$ are of interest: is it generated in degree one and what are the minimal degrees of a generating set of relations for it? For example, the question of whether the map $\Psi_{n / k}$ in Theorem 1.1 is surjective is equivalent to the question of whether its codomain $B\left(X_{n / k}, \sigma^{\prime}, \mathcal{L}_{n / k}^{\prime}\right)$ is generated by its degree-one component. None of the results referred to in the previous paragraph shows that $S\left(E^{g}, \mathcal{L}_{n / k}\right)$ is generated by its degree-one component, so one cannot expect to prove that $B\left(E^{g}, \sigma, \mathcal{L}_{n / k}\right)$ is generated by its degree-one component by tweaking the commutative arguments. We therefore develop some new methods that yield fairly complete results about $B\left(X_{n / k}, \sigma^{\prime}, \mathcal{L}_{n / k}^{\prime}\right)$ when $X_{n / k}$ is $E^{g}$ and $S^{g} E$.

\subsection{Notation}

We adopt the notation laid out at [ST94, p. 23]. For convenience we recall it.

Let $X$ be a scheme over a field $\mathbb{k}$ and let $f: \mathcal{F} \rightarrow \mathcal{G}$ be a homomorphism of $\mathcal{O}_{X}$-modules. Let $v$ be a $\mathbb{k}$-automorphism of $X$.

If $p \in X$, we write $p^{v}$ for $v(p)$. We extend this to Weil divisors in the obvious way. For example, if $D=\sum n_{p}(p)$ is a divisor on a curve, then $D^{v}:=\sum n_{p}\left(p^{v}\right)$.

We write $\mathcal{F}^{v}$ for $v^{*} \mathcal{F}=\left(v^{-1}\right)_{*} \mathcal{F}$. Thus if $D$ is a Weil divisor, $\mathcal{O}_{X}(D)^{v}=\mathcal{O}_{X}\left(D^{v^{-1}}\right)$. We write $f^{v}$ for $v^{*}(f): \mathcal{F}^{v} \rightarrow \mathcal{G}^{v}$. There is a $\mathbb{k}$-linear isomorphism

$$
H^{0}(X, \mathcal{F}) \longrightarrow H^{0}\left(X, \mathcal{F}^{v}\right)=H^{0}\left(X, \mathcal{O}_{X} \otimes_{v^{-1} \mathcal{O}_{X}} v^{-1} \mathcal{F}\right)
$$

given by $s \mapsto s^{v}:=1 \otimes s$. Notice that $s\left(p^{v}\right)=0$ if and only if $s^{v}(p)=0$. Notice too that the natural isomorphism $\operatorname{Hom}_{X}\left(\mathcal{O}_{X}, \mathcal{F}\right) \stackrel{\sim}{\longrightarrow} H^{0}(X, \mathcal{F}), f \mapsto f(1)$, satisfies $f^{v} \mapsto f(1)^{v}$.

There is a canonical map $H^{0}(X, \mathcal{F}) \otimes H^{0}(X, \mathcal{G}) \rightarrow H^{0}(X, \mathcal{F} \otimes \mathcal{G})$ which we call multiplication. If $s \in H^{0}(X, \mathcal{F})$ and $t \in H^{0}(X, \mathcal{G})$, we write $s * t$ for the image of $s \otimes t$ under the multiplication map. If $v$ is a $\mathbb{k}$-linear automorphism of $X$, then $(s * t)^{v}=s^{v} * t^{v}$, because $v^{*}$ distributes across tensor products.

If $X$ is an abelian variety and $x \in X$, we write $T_{x}: X \rightarrow X$ for the map $T_{x}(y)=x+y$. We call $T_{x}$ a translation automorphism. If $\mathcal{F}$ is an $\mathcal{O}_{X}$-module, we call $T_{x}^{*} \mathcal{F}$ a translate of $\mathcal{F}$. 
Let $X$ and $Y$ be projective $\mathbb{k}$-varieties. The Néron-Severi group of $X$ is the group $\mathrm{NS}(X):=$ $\operatorname{Pic}(X) / \operatorname{Pic}^{0}(X)$. This is a finitely generated abelian group. If $\sigma: X \rightarrow Y$ is a morphism, the inverse image functor induces a group homomorphism $\sigma^{*}: \operatorname{Pic}(Y) \rightarrow \operatorname{Pic}(X)$ that descends to a homomorphism $\mathrm{NS}(Y) \rightarrow \mathrm{NS}(X)$, and hence to $\mathrm{NS}(Y)_{\mathbb{C}} \rightarrow \mathrm{NS}(X)_{\mathbb{C}}$. The last homomorphism can be represented by a matrix with entries in $\mathbb{Z}$. If $\sigma \in \operatorname{Aut}(X)$, we call $\sigma^{*}$ quasi-unipotent if all its eigenvalues in $\mathbb{C}$ are roots of unity.

\subsection{The twisted homogeneous coordinate rings $B(X, \sigma, \mathcal{L})$}

The rings $B(X, \sigma, \mathcal{L})$ we are about to define were introduced by Artin, Tate and Van den Bergh in [ATVdB90], and independently by Feigin and Odesskii in [FO89, OF89], as a device to understand graded algebras that map to them. That understanding is obtained through Theorem 2.4 and Corollary 2.8.

Proposition 2.1. Let $\mathbb{k}$ be a field. There is a contravariant functor $(X, \sigma, \mathcal{L}) \rightsquigarrow B(X, \sigma, \mathcal{L})$ from the category of triples consisting of a $\mathbb{k}$-scheme $X$, a $\mathbb{k}$-automorphism $\sigma: X \rightarrow X$ and an invertible $\mathcal{O}_{X^{-}}$ module $\mathcal{L}$, to the category of graded $\mathbb{k}$-algebras.

This needs some explanation.

A morphism of triples is a pair $(f, u):(X, \sigma, \mathcal{L}) \rightarrow\left(X^{\prime}, \sigma^{\prime}, \mathcal{L}^{\prime}\right)$ consisting of a $\mathbb{k}$-morphism $f: X \rightarrow X^{\prime}$ such that $f \sigma=\sigma^{\prime} f$ and a homomorphism $u: f^{*} \mathcal{L}^{\prime} \rightarrow \mathcal{L}$.

As a graded vector space, the $\mathbb{k}$-algebra $B(X, \sigma, \mathcal{L})$ is

$$
\bigoplus_{i=0}^{\infty} H^{0}\left(X, \mathcal{L}_{i}\right)
$$

where

$$
\mathcal{L}_{i}:=\mathcal{L} \otimes \mathcal{L}^{\sigma} \otimes \cdots \otimes \mathcal{L}^{\sigma^{i-1}}
$$

The product $x \cdot y$ of $x \in H^{0}\left(X, \mathcal{L}_{i}\right)$ and $y \in H^{0}\left(X, \mathcal{L}_{j}\right)$ is $x * y^{\sigma^{i}}-$ that is, the image of $x \otimes y^{\sigma^{i}}$ under the natural multiplication map

$$
H^{0}\left(X, \mathcal{L}_{i}\right) \otimes H^{0}\left(X, \mathcal{L}_{j}^{\sigma^{i}}\right) \longrightarrow H^{0}\left(X, \mathcal{L}_{i} \otimes \mathcal{L}_{j}^{\sigma^{i}}\right)
$$

We call $B(X, \sigma, \mathcal{L})$ a twisted homogeneous coordinate ring. The terminology is motivated and justified by Theorem 2.4 .

\subsubsection{Isomorphisms and anti-isomorphisms}

The next two results are probably known to the experts.

Proposition 2.2. $B\left(X, \sigma^{-1}, \mathcal{L}\right)^{\mathrm{op}} \cong B(X, \sigma, \mathcal{L})$, where $(\cdot)^{\mathrm{op}}$ denotes the opposite ring.

Proof. We write $B^{\prime}:=B\left(X, \sigma^{-1}, \mathcal{L}\right)$ and $B:=B(X, \sigma, \mathcal{L})$, and denote the multiplication maps by $\mu: B \otimes B \rightarrow B$ and $\mu^{\prime}: B^{\prime} \otimes B^{\prime} \rightarrow B^{\prime}$. We will prove the result by defining a degree-preserving $\mathbb{k}$-linear isomorphism $\varphi: B \rightarrow B^{\prime}$ with the property that $\varphi \circ \mu(x \otimes y)=\mu^{\prime}(\varphi(y) \otimes \varphi(x))$ for all homogeneous $x$ and $y$ in $B$.

Let $\mathcal{L}_{n}^{\prime}=\mathcal{L} \otimes \mathcal{L}^{\sigma^{-1}} \otimes \ldots \otimes \mathcal{L}^{\sigma^{-n+1}}$. The degree- $n$ component of $B^{\prime}$ is $B_{n}^{\prime}:=H^{0}\left(X, \mathcal{L}_{n}^{\prime}\right)$.

Since $\mathcal{L}_{n}^{\prime}=\left(\mathcal{L}_{n}\right)^{\sigma^{-n+1}}$, there is a $\mathbb{k}$-linear isomorphism $\varphi_{n}: H^{0}\left(X, \mathcal{L}_{n}\right) \rightarrow H^{0}\left(X, \mathcal{L}_{n}^{\prime}\right)$ given by $\varphi(x)=x^{\sigma^{-n+1}}$. If $x \in H^{0}\left(X, \mathcal{L}_{m}\right)$ and $y \in H^{0}\left(X, \mathcal{L}_{n}\right)$, then

$$
\varphi \circ \mu(x \otimes y)=\left(x * y^{\sigma^{m}}\right)^{\sigma^{-m-n+1}} .
$$


On the other hand,

$$
\mu^{\prime}(\varphi(y) \otimes \varphi(x))=y^{\sigma^{-n+1}} *\left(x^{\sigma^{-m+1}}\right)^{\sigma^{-n}}
$$

The result now follows from the fact that the inverse image commutes with the tensor product (see $\S 2.2)$.

Proposition 2.3. Let $(X, \sigma, \mathcal{L})$ be a triple. If $\mu: X \rightarrow X$ is a $\mathbb{k}$-automorphism, there is an isomorphism

$$
B(X, \sigma, \mathcal{L}) \stackrel{\sim}{\longrightarrow} B\left(X, \mu^{-1} \sigma \mu, \mu^{*} \mathcal{L}\right)
$$

sending $s \in H^{0}(X, \mathcal{L})=B(X, \sigma, \mathcal{L})_{1}$ to $\mu^{*}(s)=s^{\mu} \in H^{0}\left(X, \mu^{*} \mathcal{L}\right)$.

Proof. This is an immediate consequence of Proposition 2.1, because $\left(\mu\right.$, id) $:\left(X, \mu^{-1} \sigma \mu, \mu^{*} \mathcal{L}\right) \rightarrow$ $(X, \sigma, \mathcal{L})$ is an isomorphism of triples.

\subsection{2. $\sigma$-ampleness}

When $X$ is Noetherian we say $\mathcal{L}$ is $\sigma$-ample if for every coherent $\mathcal{O}_{X}$-module $\mathcal{F}$,

$$
H^{q}\left(X, \mathcal{F} \otimes \mathcal{L}_{i}\right)=0
$$

for all $q \geq 1$ and all $i \gg 0$. When $\sigma$ is the identity, this becomes the traditional definition of ampleness.

2.3.3. The Artin-Van den Bergh theorem and the functor $\Gamma_{*}: \operatorname{Qcoh}(X) \rightarrow \operatorname{Gr}(B(X, \sigma, \mathcal{L}))$

Following [AVdB90, AZ94], we define the auto-equivalence $s: \mathrm{Qcoh}(X) \rightarrow \mathrm{Qcoh}(X)$ by the formula

$$
s:=\mathcal{L} \otimes \sigma^{*}(\cdot), \quad \text { that is, } s(\mathcal{M})=\mathcal{L} \otimes \sigma^{*} \mathcal{M}=\mathcal{L} \otimes \mathcal{M}^{\sigma} .
$$

We write $s^{0}$ for the identity functor on $Q \operatorname{coh}(X)$. Now define the graded vector space

$$
M:=\bigoplus_{n \in \mathbb{Z}} H^{0}\left(X, s^{n}(\mathcal{M})\right)=\bigoplus_{n \in \mathbb{Z}} \operatorname{Hom}_{\mathcal{O}_{X}}\left(\mathcal{O}_{X}, s^{n}(\mathcal{M})\right)=\bigoplus_{n \in \mathbb{Z}} H^{0}\left(X, \mathcal{L}_{n} \otimes \mathcal{M}^{\sigma^{n}}\right) .
$$

Let $b \in B(X, \sigma, \mathcal{L})_{i}$ and $m \in M_{j}=H^{0}\left(X, \mathcal{L}_{j} \otimes \mathcal{M}^{\sigma^{j}}\right)$. Since $m$ is a homomorphism $\mathcal{O}_{X} \rightarrow \mathcal{L}_{j} \otimes \mathcal{M}^{\sigma^{j}}$,

$$
s^{i}(m): s^{i}\left(\mathcal{O}_{X}\right)=\mathcal{L}_{i} \longrightarrow s^{i}\left(\mathcal{L}_{j} \otimes \mathcal{M}^{\sigma^{j}}\right)=\mathcal{L}_{i} \otimes\left(\mathcal{L}_{j} \otimes \mathcal{M}^{\sigma^{j}}\right) \sigma^{\sigma^{i}}=\mathcal{L}_{i+j} \otimes \mathcal{M}^{\sigma^{i+j}} .
$$

Since $b$ is a homomorphism $\mathcal{O}_{X} \rightarrow \mathcal{L}_{i}$, we may define

$$
b \cdot m:=s^{i}(m) \circ b .
$$

This formula gives $M$ the structure of a graded left $B(X, \sigma, \mathcal{L})$-module. We define $\Gamma_{*}$ by

$$
\Gamma_{*} \mathcal{M}:=\bigoplus_{n \in \mathbb{Z}} H^{0}\left(X, \mathcal{L}_{n} \otimes \mathcal{M}^{\sigma^{n}}\right)
$$

with this graded module structure. Sometimes we abuse notation and write $\Gamma_{*}$ for the composition

$$
\operatorname{Qcoh}(X) \longrightarrow \operatorname{\Gamma _{*}} \operatorname{Gr}(B(X, \sigma, \mathcal{L})) \longrightarrow \operatorname{QGr}(B(X, \sigma, \mathcal{L})) .
$$

Theorem 2.4 (Artin-Van den Bergh [AVdB90, Theorems 1.3 and 1.4]). If $X$ is a projective $\mathbb{k}$-scheme and $\mathcal{L}$ is $\sigma$-ample, then $B(X, \sigma, \mathcal{L})$ is a finitely generated left Noetherian $\mathbb{k}$-algebra, and the functor $\Gamma_{*}$ provides an equivalence of categories

$$
\operatorname{Qcoh}(X) \equiv \operatorname{QGr}(B(X, \sigma, \mathcal{L})) .
$$


By [Kee00, Corollary 5.1], $\mathcal{L}$ is $\sigma$-ample if and only if it is $\sigma^{-1}$-ample. Thus, by Proposition 2.2, in the context of Theorem $2.4 B(X, \sigma, \mathcal{L})$ is right Noetherian too [Kee00, Corollary 5.3].

Theorem 2.5 (Keeler [Kee00, Theorems 1.2 and 1.4]). Let $\sigma$ be an automorphism of a projective scheme $X$ over an algebraically closed field $\mathbb{k}$. The following conditions are equivalent:

(1) There is a $\sigma$-ample invertible $\mathcal{O}_{X}$-module.

(2) Every ample invertible $\mathcal{O}_{X}$-module is $\sigma$-ample.

(3) The action of $\sigma^{*}$ on $\mathrm{NS}(X)_{\mathbb{C}}$ is quasi-unipotent.

Furthermore, if one of those conditions holds, then

(4) the $G K$-dimension of $B(X, \sigma, \mathcal{L})$ is an integer for every ample $\mathcal{L}$ and

(5) $B(X, \sigma, \mathcal{L})$ is right and left Noetherian for every ample $\mathcal{L}$.

The next result applies to $X=E^{g}$ and all translation automorphisms $\sigma: E^{g} \rightarrow E^{g}$.

Corollary 2.6. Let $X$ be a projective scheme over an algebraically closed field $\mathbb{k}$ and $G$ an algebraic group over $\mathbb{k}$ that acts on $X$. If $\sigma \in G$, then every ample invertible $\mathcal{O}_{X}$-module is $\sigma$-ample.

Proof. By Theorem 2.5 it suffices to show that $\sigma^{*}$ is quasi-unipotent.

The Picard functor is representable by a scheme Pic $(X)$ [Mur64, II.15] which is acted upon by $G$. Since $G$ has finitely many connected components (as all algebraic groups do), some power of $\sigma$, say $\sigma^{r}$, belongs to the connected component $G^{0}$ that contains the identity. The action of $G^{0}$ sends each connected component of $\operatorname{Pic}(X)$ to itself and hence acts trivially on $\operatorname{NS}(X)=\operatorname{Pic}(X) / \mathrm{Pic}^{0}(X)$. In particular, $\left(\sigma^{*}\right)^{r}$ acts trivially on $\mathrm{NS}(X)_{\mathbb{C}}$, so the action of $\sigma^{*}$ on $\operatorname{NS}(X)_{\mathbb{C}}$ is quasi-unipotent.

Corollary 2.7. Let $\Sigma$ be a finite group acting as group automorphisms of an abelian variety A over an algebraically closed field $\mathbb{k}$. If $\sigma: A \rightarrow A$ is translation by a point that is fixed by $\Sigma$, then $\sigma$ descends to an automorphism $\sigma^{\prime}$ of $A / \Sigma$ having the property that every ample invertible module over $A / \Sigma$ is $\sigma^{\prime}$-ample.

Proof. The set $A^{\Sigma}$ consisting of points fixed by $\Sigma$ is an algebraic subgroup of $A$. It acts on $A$ by translation automorphisms and each such automorphism descends to an automorphism of $A / \Sigma$. Since $A / \Sigma$ is a projective variety, the result follows from Corollary 2.6 with $G=A^{\Sigma}$.

\subsection{Using the rings $B(X, \sigma, \mathcal{L})$}

If $J$ is a graded ideal in a finitely generated $\mathbb{N}$-graded algebra $A$ over a field $\mathbb{k}$, the three natural functors between the categories $\operatorname{Gr}(A)$ and $\operatorname{Gr}(A / J)$ induce functors

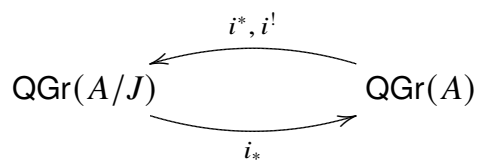

between the quotient categories such that $i_{*}$ is a fully faithful embedding whose essential image is closed under subobjects and quotients, $i^{*}$ is left adjoint to $i_{*}$ and $i^{!}$is right adjoint to $i_{*}$ (see [Smi16, VdB01]). The functors $i^{*}$ and $i_{*}$ behave like the inverse and direct image functors associated to a closed immersion of one scheme in another. Thus, the next result says in effect that the noncommutative scheme with homogeneous coordinate ring $A$ has a closed subscheme isomorphic to $X$. In this paper, we will show that this happens when $A$ is $Q_{n, k}(E, \tau)$ and $X$ is its characteristic variety, provided that that characteristic variety is a product or symmetric product of copies of $E$. 
Corollary 2.8. Let $A$ be an $\mathbb{N}$-graded $\mathbb{k}$-algebra. Assume the hypotheses in Theorem 2.4 hold. If there is a surjective homomorphism $A \rightarrow B(X, \sigma, \mathcal{L})$, then there are functors

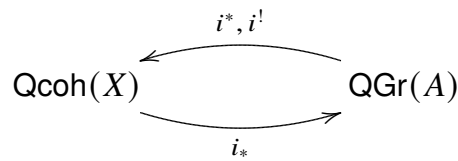

in which $i_{*}$ is a fully faithful functor whose essential image is closed under subobjects and quotients, $i^{*}$ is left adjoint to $i_{*}$ and $i^{*}$ is right adjoint to $i_{*}$.

In [ATVdB90, §3.17, Proposition 3.20], Artin, Tate and Van den Bergh describe a procedure that associates to a fairly general graded $\mathbb{k}$-algebra $A$ a canonical algebra $B$ and a canonical homomorphism of graded algebras $A \rightarrow B$. In general, $B$ might not be of the form $B(X, \sigma, \mathcal{L})$. But in a number of important situations it is.

The next result, which uses ideas in [ATVdB90] and [FO89, p. 8], will be applied to $A=Q_{n, k}(E, \tau)$. In it we view elements of $V^{\otimes 2}$ as forms of bidegree $(1,1)$ on the product of projective spaces $\mathbb{P}\left(V^{*}\right) \times$ $\mathbb{P}\left(V^{*}\right)$.

Proposition 2.9. Let $T V$ denote the tensor algebra on a finite-dimensional $\mathbb{k}$-vector space $V$ and let $A=T V /(R)$ be the quotient by the ideal generated by a subspace $R \subseteq V^{\otimes 2}$. Let $X$ be a $\mathbb{k}$-scheme, $\sigma \in \operatorname{Aut}_{\mathbb{k}}(X), \Gamma_{\sigma}$ the graph of $\sigma, f: X \rightarrow \mathbb{P}\left(V^{*}\right)$ a morphism and $\mathcal{L}=f^{*} \mathcal{O}_{\mathbb{P}\left(V^{*}\right)}(1)$. If $R$ vanishes on $(f \times f)\left(\Gamma_{\sigma}\right)$, then the canonical linear map $V \rightarrow H^{0}(X, \mathcal{L})$ extends to a $\mathbb{k}$-algebra homomorphism $\varphi: A \rightarrow B(X, \sigma, \mathcal{L})$.

Proof. Since $V=H^{0}\left(\mathbb{P}\left(V^{*}\right), \mathcal{O}_{\mathbb{P}\left(V^{*}\right)}(1)\right)$, there is a canonical linear map $V \rightarrow H^{0}(X, \mathcal{L})=B(X, \sigma, \mathcal{L})_{1}$. This map extends in a unique way to a homomorphism $\varphi: T V \rightarrow B(X, \sigma, \mathcal{L})$. An element $w \in V \otimes V=$ $H^{0}(X \times X, \mathcal{L} \otimes \mathcal{L})$ is in the kernel of $\varphi$ if and only if it vanishes on $(f \times f)\left(\Gamma_{\sigma}\right)$. Since elements of $R$ vanish on this graph by hypothesis, $R \subseteq \operatorname{ker}(\varphi)$. The result follows.

Proposition 2.10. Let $Y$ be a projective $\mathbb{k}$-scheme, $\sigma$ a $\mathbb{k}$-automorphism of $Y$ and $\mathcal{L}$ a base-point free invertible $\mathcal{O}_{Y}$-module. Let $\Phi: Y \rightarrow \mathbb{P}\left(H^{0}(Y, \mathcal{L})^{*}\right)$ be the morphism associated to the complete linear system $|\mathcal{L}|$ and let $X=\Phi(Y)$. There is a factorisation $\Phi=i \circ f$ where $i: X \rightarrow \mathbb{P}\left(H^{0}(Y, \mathcal{L})^{*}\right)$ is the inclusion and $f: Y \rightarrow X$ is obtained by restricting the codomain of $\Phi$. Let $\mathcal{L}^{\prime}=\left.\mathcal{O}(1)\right|_{X}$. If $\sigma^{\prime}: X \rightarrow X$ is an automorphism such that $f \sigma=\sigma^{\prime} f$, then the canonical map $H^{0}\left(X, \mathcal{L}^{\prime}\right) \rightarrow H^{0}(Y, \mathcal{L})$ extends to a homomorphism of graded rings

$$
\varphi: B\left(X, \sigma^{\prime}, \mathcal{L}^{\prime}\right) \longrightarrow B(Y, \sigma, \mathcal{L})
$$

Proof. Since $\mathcal{L}$ is generated by its global sections, $\mathcal{L} \cong \Phi^{*} \mathcal{O}(1)$. Since $\Phi^{*} \mathcal{O}(1)=f^{*} i^{*} \mathcal{O}(1)=f^{*} \mathcal{L}^{\prime}$, there is an isomorphism $u: f^{*} \mathcal{L}^{\prime} \rightarrow \mathcal{L}$. We therefore obtain a morphism of triples $(f, u):(Y, \sigma, \mathcal{L}) \rightarrow$ $\left(X, \sigma^{\prime}, \mathcal{L}^{\prime}\right)$ and hence, by functoriality of the $B$-construction, a homomorphism $\varphi$ as claimed.

In Corollary 3.6, we apply Propositions 2.9 and 2.10 to obtain homomorphisms

$$
Q_{n, k}(E, \tau) \stackrel{\Psi}{\longrightarrow} B\left(X_{n / k}, \sigma^{\prime}, \mathcal{L}_{n / k}^{\prime}\right) \longrightarrow B\left(E^{g}, \sigma, \mathcal{L}_{n / k}\right) .
$$

The following questions then become relevant:

○ Is $\mathcal{L}_{n / k}^{\prime}$ a $\sigma^{\prime}$-ample sheaf?

○ Is $B\left(X_{n / k}, \sigma^{\prime}, \mathcal{L}_{n / k}^{\prime}\right)$ generated in degree one - that is, is $\Psi$ surjective?

$\circ$ Are the relations for $B\left(X_{n / k}, \sigma^{\prime}, \mathcal{L}_{n / k}^{\prime}\right)$ generated in degrees 2 and 3 ?

We answer these in the affirmative when $X_{n / k}$ is $E^{g}$ and $S^{g} E$. 


\subsection{Generators and relations for $B(X, \sigma, \mathcal{L})$}

We assume that $\mathbb{k}$ is an algebraically closed field, $X$ is a projective $\mathbb{k}$-scheme and $\sigma: X \rightarrow X$ is a $\mathbb{k}$-automorphism.

In this section we use the following notation:

$$
\begin{aligned}
\mathcal{L} & :=\text { an ample invertible } \mathcal{O}_{X} \text {-module generated by its global sections } \\
\mathcal{M}=\mathcal{M}_{m} & :=\sigma^{*} \mathcal{L} \otimes \cdots \otimes\left(\sigma^{*}\right)^{m} \mathcal{L} \\
\mathcal{N}=\mathcal{N}_{m} & :=\left(\sigma^{m+1}\right)^{*} \mathcal{L} \\
\mathcal{K}=\mathcal{K}_{m} & :=\operatorname{ker}\left(H^{0}\left(X, \mathcal{N}_{m}\right) \otimes \mathcal{O}_{X} \rightarrow \mathcal{N}_{m}\right) \\
\mathcal{G}=\mathcal{G}_{m} & :=\mathcal{M}_{m} \otimes \mathcal{K}_{m}=\operatorname{ker}\left(\mathcal{M}_{m} \otimes H^{0}\left(X, \mathcal{N}_{m}\right) \rightarrow \mathcal{M}_{m} \otimes \mathcal{N}_{m}\right) \\
R(\mathcal{M}, \mathcal{N}) & :=\operatorname{ker}\left(H^{0}(X, \mathcal{M}) \otimes H^{0}(X, \mathcal{N}) \rightarrow H^{0}(X, \mathcal{M} \otimes \mathcal{N})\right)
\end{aligned}
$$

The notation $R(\mathcal{M}, \mathcal{N})$ is taken from [Mum70, SS92].

There is a natural map

$$
H^{0}(X, \mathcal{L}) \otimes R\left(\mathcal{M}_{m}, \mathcal{N}_{m}\right) \rightarrow R\left(\mathcal{L} \otimes \mathcal{M}_{m}, \mathcal{N}_{m}\right)
$$

that fits into the following commutative diagram with exact rows:

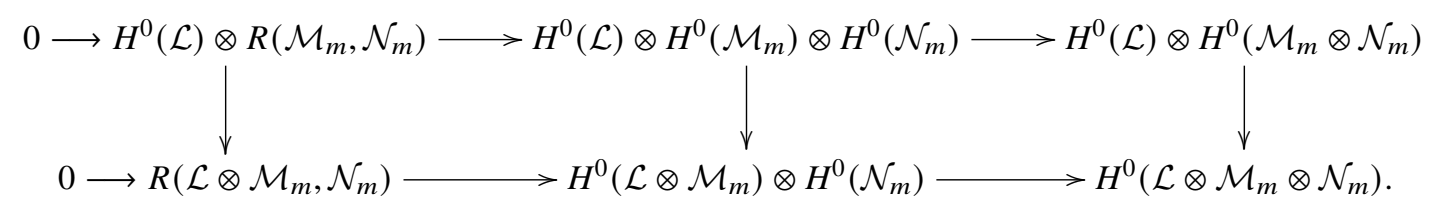

The next result is a small extension of [SS92, Lemma 3.7].

Lemma 2.11. Let $X$ be a connected projective $\mathbb{k}$-scheme - that is, $H^{0}\left(X, \mathcal{O}_{X}\right)=\mathbb{k}, \sigma: X \rightarrow X$ $a \mathbb{k}$-automorphism and $\mathcal{L}$ an ample invertible $\mathcal{O}_{X}$-module generated by its global sections. Suppose $B=B(X, \sigma, \mathcal{L})$ is generated as a $\mathbb{k}$-algebra by $B_{1}$. Write $B=T\left(B_{1}\right) / J$, where $T\left(B_{1}\right)$ is the tensor algebra on $B_{1}$. Let $J_{i}=J \cap B_{1}^{\otimes i}$. The ideal $J$ is generated by $J_{2}+\cdots+J_{\ell}$ if and only if the map in (2.3) is surjective for all $m \geq \ell-1$.

Proof. The degree- $r$ component of $T=T\left(B_{1}\right)$ is $T_{r}=B_{1}^{\otimes r}$. Clearly, $J$ is generated by $J_{2}+\cdots+J_{\ell}$ if and only if $J_{r+1}=T_{1} J_{r}+J_{r} T_{1}$ for all $r \geq \ell-$ that is, if and only if

$$
\frac{J_{r+1}}{J_{r} T_{1}}=\frac{T_{1} J_{r}+J_{r} T_{1}}{J_{r} T_{1}}
$$

for all $r \geq \ell$.

We will now reformulate this, but first, to be consistent with the definitions of $\mathcal{L}, \mathcal{M}, \mathcal{N}$, we set $m=r-1$ so that $J_{r+1}=J_{m+2}$.

There is a commutative diagram

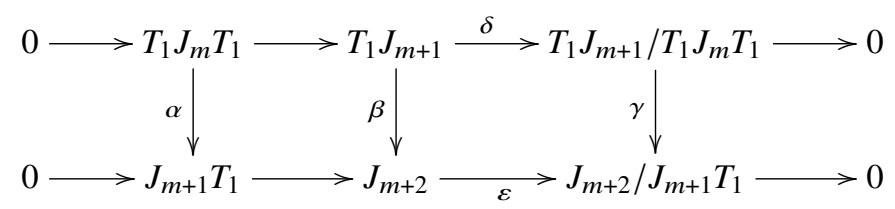

in which $\alpha$ and $\beta$ are the natural inclusions, the rows are exact and $\gamma$ is the unique linear map such that 
$\varepsilon \beta=\gamma \delta$. Clearly

$$
\operatorname{im}(\gamma)=\operatorname{im}(\gamma \delta)=\operatorname{im}(\varepsilon \beta)=\frac{T_{1} J_{m+1}+J_{m+1} T_{1}}{J_{m+1} T_{1}} .
$$

Thus, $\gamma$ is surjective if and only if $J_{m+2}=T_{1} J_{m+1}+J_{m+1} T_{1}$.

Hence $J$ is generated by $J_{2}+\cdots+J_{\ell}$ if and only if $\gamma$ is surjective for all $m \geq \ell-1$. Since $T_{1}=B_{1}=H^{0}(\mathcal{L})$, the right-hand square in the commutative diagram (2.4) is canonically isomorphic to the diagram

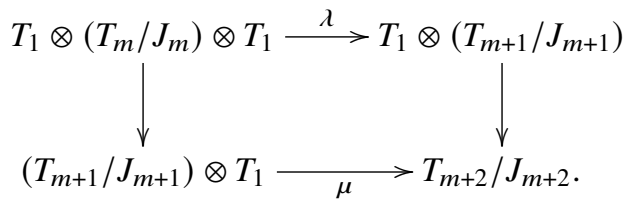

Thus the map in (2.3) is surjective if and only if the induced map $\operatorname{ker} \lambda \rightarrow \operatorname{ker} \mu$ is surjective. Here we have

$$
\begin{aligned}
\operatorname{ker}(\lambda) & =\operatorname{ker}\left(\frac{T_{1} \otimes T_{m} \otimes T_{1}}{T_{1} \otimes J_{m} \otimes T_{1}} \longrightarrow \frac{T_{1} \otimes T_{m+1}}{T_{1} \otimes J_{m+1}}\right) \\
& =\operatorname{ker}\left(\frac{T_{m+2}}{T_{1} J_{m} T_{1}} \longrightarrow \frac{T_{m+2}}{T_{1} J_{m+1}}\right) \\
& =\frac{T_{1} J_{m+1}}{T_{1} J_{m} T_{1}}
\end{aligned}
$$

and

$$
\operatorname{ker}(\mu)=\operatorname{ker}\left(\frac{T_{m+2}}{J_{m+1} T_{1}} \longrightarrow \frac{T_{m+2}}{J_{m+2}}\right)=\frac{J_{m+2}}{J_{m+1} T_{1}}
$$

and these equalities identify the map $\operatorname{ker} \lambda \rightarrow \operatorname{ker} \mu$ with $\gamma$. This completes the proof.

Lemma 2.12. If $B(X, \sigma, \mathcal{L})$ is generated in degree one, then its relations are generated in degree $\leq \ell$ if and only if the multiplication map

$$
H^{0}(X, \mathcal{L}) \otimes H^{0}\left(X, \mathcal{G}_{m}\right) \longrightarrow H^{0}\left(X, \mathcal{L} \otimes \mathcal{G}_{m}\right)
$$

is onto for all $m \geq \ell-1$.

Proof. Fix an integer $m$. By Lemma 2.11, it suffices to show that the map

$$
H^{0}(X, \mathcal{L}) \otimes R(\mathcal{M}, \mathcal{N}) \longrightarrow R(\mathcal{L} \otimes \mathcal{M}, \mathcal{N})
$$

is onto if and only if the map $H^{0}(X, \mathcal{L}) \otimes H^{0}(X, \mathcal{G}) \longrightarrow H^{0}(X, \mathcal{L} \otimes \mathcal{G})$ is onto.

There are exact sequences $0 \rightarrow \mathcal{G} \rightarrow \mathcal{M} \otimes H^{0}(X, \mathcal{N}) \rightarrow \mathcal{M} \otimes \mathcal{N} \rightarrow 0$ and

$$
0 \rightarrow \mathcal{L} \otimes \mathcal{G} \rightarrow \mathcal{L} \otimes \mathcal{M} \otimes H^{0}(X, \mathcal{N}) \rightarrow \mathcal{L} \otimes \mathcal{M} \otimes \mathcal{N} \rightarrow 0,
$$

and therefore exact sequences

$$
0 \rightarrow H^{0}(X, \mathcal{G}) \rightarrow H^{0}(X, \mathcal{M}) \otimes H^{0}(X, \mathcal{N}) \rightarrow H^{0}(X, \mathcal{M} \otimes \mathcal{N})
$$

and

$$
0 \rightarrow H^{0}(X, \mathcal{L} \otimes \mathcal{G}) \rightarrow H^{0}(X, \mathcal{L} \otimes \mathcal{M}) \otimes H^{0}(\mathcal{N}) \rightarrow H^{0}(X, \mathcal{L} \otimes \mathcal{M} \otimes \mathcal{N})
$$


Thus, there are canonical isomorphisms $R(\mathcal{M}, \mathcal{N}) \cong H^{0}(X, \mathcal{G})$ and $R(\mathcal{L} \otimes \mathcal{M}, \mathcal{N}) \cong H^{0}(X, \mathcal{L} \otimes \mathcal{G})$; it follows that (2.3) is onto if and only if the multiplication map $H^{0}(X, \mathcal{L}) \otimes H^{0}(X, \mathcal{G}) \longrightarrow H^{0}(X, \mathcal{L} \otimes \mathcal{G})$ is onto.

\subsection{Point modules for $B(X, \sigma, \mathcal{L})$}

The 'simplest part' of the representation theory of a noncommutative algebra consists of its 1-dimensional modules. The 'simplest part' of the graded representation theory of a connected graded $\mathbb{K}$-algebra, say $A$, consists of its point modules: a point module for $A$ is a cyclic graded left $A$-module $M=M_{0} \oplus M_{1} \oplus \cdots$ such that $\operatorname{dim}_{\mathbb{k}}\left(M_{i}\right)=1$ for all $i \geq 0$ (see [ATVdB91] and [OF89, p. 208]).

The next result was known to Artin, Tate and Van den Bergh [ATVdB90] and to Feigin and Odesskii [OF89] sometime in the late 1980s, but it was not recorded explicitly.

Proposition 2.13. Let $\mathcal{O}_{p}$ be the skyscraper sheaf at a closed point $p \in X$. If $\mathcal{L}$ is generated by its global sections, then

$$
M_{p}:=\left(\Gamma_{*} \mathcal{O}_{p}\right)_{\geq 0}=\bigoplus_{n=0}^{\infty} H^{0}\left(X, \mathcal{O}_{p}^{\sigma^{n}}\right)
$$

is a point module for $B(X, \sigma, \mathcal{L})$ and

$$
\left(M_{p}\right)_{\geq 1}(1) \cong M_{\sigma^{-1} p}
$$

An element $b \in B(X, \sigma, \mathcal{L})_{1}$ annihilates the degree- $n$ component of $M_{p}$ if and only if $b\left(\sigma^{-n} p\right)=0$.

Proof. By definition,

$$
\Gamma_{*} \mathcal{O}_{p}=\bigoplus_{n \in \mathbb{Z}} H^{0}\left(X, \mathcal{L}_{n} \otimes \mathcal{O}_{p}^{\sigma^{n}}\right) \cong \bigoplus_{n \in \mathbb{Z}} H^{0}\left(X, \mathcal{O}_{\sigma^{-n} p}\right) .
$$

A section $b \in B(X, \sigma, \mathcal{L})_{1}=H^{0}(X, \mathcal{L})$ annihilates the degree- $n$ component of $\Gamma_{*} \mathcal{O}_{p}$ if and only if $b\left(\sigma^{-n} p\right)=0$. Since $\mathcal{L}$ is generated by its global sections, for each $x \in X$ there is some $b \in B(X, \sigma, \mathcal{L})_{1}$ such that $b(x) \neq 0$. It follows that $M_{p}$ is generated by its degree-zero component as a $B(X, \sigma, \mathcal{L})$ module. Since $\operatorname{dim}_{\mathbb{K}}\left(\left(M_{p}\right)_{i}\right)=1$ for all $i \geq 0, M_{p}$ is a point module for $B(X, \sigma, \mathcal{L})$.

The degree- $i$ component of $M_{\sigma^{-1} p}$ is $H^{0}\left(X, \mathcal{O}_{\sigma^{-i}\left(\sigma^{-1} p\right)}\right)=H^{0}\left(X, \mathcal{O}_{\left.\sigma^{-i-1} p\right)}\right)=\left(M_{p}\right)_{i+1}$. It follows that $\left(M_{p}\right)_{\geq 1}(1) \cong M_{\sigma^{-1}}$.

\subsubsection{Remark}

When $X$ is projective and $B(X, \mathrm{id}, \mathcal{L})$ is finitely generated, each point module for $B(X, \mathrm{id}, \mathcal{L})$ is isomorphic in $\operatorname{QGr}(B(X, \mathrm{id}, \mathcal{L}))$ to one of the $M_{p}$ in Proposition 2.13. We will now prove this claim.

First, [The18, Tag 01Q0] implies that the image of the canonical morphism

$$
f: X \longrightarrow \operatorname{Proj}(B(X, \mathrm{id}, \mathcal{L}))
$$

is dense; since $X$ is projective, the image is closed, so the morphism is onto. The morphism $f$ has the property that $f^{-1}\left(D_{+}(s)\right)=X_{s}$ for all homogeneous $s \in B(X, \mathrm{id}, \mathcal{L})_{+}$, and this implies that $f(p)=\operatorname{Ann}\left(M_{p}\right)$ for all closed points $p \in X$, where the annihilator $\operatorname{Ann}\left(M_{p}\right)$ inside $B(X, \mathrm{id}, \mathcal{L})$ is a homogeneous ideal that is maximal among those not containing $B(X, \mathrm{id}, \mathcal{L})_{+}$and hence is regarded as a point of $\operatorname{Proj}(B(X, \mathrm{id}, \mathcal{L}))$.

Now let $N$ be a point module for $B(X, \mathrm{id}, \mathcal{L})$. Since $B(X, \mathrm{id}, \mathcal{L})$ is finitely generated, $N$ admits a subquotient isomorphic to $B(X, \mathrm{id}, \mathcal{L}) / \mathrm{m}$ shifted by some degree $d \in \mathbb{Z}$, where $\mathrm{m}$ is a point of $\operatorname{Proj}(B(X, \mathrm{id}, \mathcal{L}))$ regarded as a homogeneous ideal of $B(X, \mathrm{id}, \mathcal{L})$. The surjectivity of $(2.7)$ implies that $\mathrm{m}=f(p)$ for some $p \in X$, whence $\mathrm{m}=\operatorname{Ann}\left(M_{p}\right)$. Therefore $M_{p}$ is isomorphic to $B(X, \mathrm{id}, \mathcal{L}) / \mathrm{m}$ and its degree shift by $d$ is a subquotient of $N$. Since both $M_{p}$ and $N$ are point modules, $d \leq 0$ 
and $M_{p}(d) \cong\left(M_{\sigma^{-d}(p)}\right)_{\geq-d}$ is a submodule of $N$. It follows that $N$ is isomorphic to $M_{\sigma^{-d}(p)}$ in $\operatorname{QGr}(B(X, \mathrm{id}, \mathcal{L}))$.

\section{The algebras $Q_{n, k}(E, \tau)$}

As always, $n$ and $k$ are relatively prime integers such that $n>k \geq 1$.

\subsection{Some notation and results from [CKS19b]}

3.1.1.

Fix $\eta \in \mathbb{C}$ lying in the upper half-plane. Let $\Lambda=\mathbb{Z}+\mathbb{Z} \eta$ and $E=\mathbb{C} / \Lambda$. We will usually view $E$ as an elliptic curve. If $r$ is a positive integer, we write $E[r]$ for the $r$-torsion subgroup of $E$. It equals $\frac{1}{r} \Lambda / \Lambda$ and thus is isomorphic to $\mathbb{Z}_{r} \times \mathbb{Z}_{r}$.

We fix a point $\tau \in \mathbb{C}$ and use the symbol $\tau$ to denote the image of $\tau$ in $E$ and the translation automorphisms $\mathbb{C} \rightarrow \mathbb{C}$ and $E \rightarrow E$ given by the formula $z \mapsto z+\tau$. The meaning of $\tau$ will always be clear from the context.

\subsection{2.}

At different times we give the degree-one component of $Q_{n, k}(E, \tau)$ different interpretations as:

(1) an anonymous vector space $V$ with basis $\left\{x_{\alpha} \mid \alpha \in \mathbb{Z}_{n}\right\}$,

(2) a space $\Theta_{n}(\Lambda)$ of theta functions in one variable with basis $\left\{\theta_{\alpha}(z) \mid \alpha \in \mathbb{Z}_{n}\right\}$,

(3) a space $\Theta_{n / k}(\Lambda)$ of theta functions in $g$ variables with basis $\left\{w_{\alpha}(z) \mid \alpha \in \mathbb{Z}_{n}\right\}$,

(4) $H^{0}\left(E^{g}, \mathcal{L}_{n / k}\right)$, where $\mathcal{L}_{n / k}$ is the invertible $\mathcal{O}_{E^{g}}$-module defined by equation (3.3) or

(5) $H^{0}\left(X_{n / k}, \mathcal{L}_{n / k}^{\prime}\right)$, where $X_{n / k}$ and $\mathcal{L}_{n / k}^{\prime}$ are defined later.

See [CKS19b, §5.3] for the relations between these interpretations.

\subsubsection{Negative continued fractions}

If $a, b, \ldots, c$ are integers $\geq 2$, we write

$$
[a, b, \ldots, c]:=a-\frac{1}{b-\frac{1}{\ddots \cdot-\frac{1}{c}}} .
$$

There is a unique integer $g \geq 1$ and a unique sequence of integers $n_{1}, \ldots, n_{g}$, all $\geq 2$, such that

$$
\frac{n}{k}=\left[n_{1}, \ldots, n_{g}\right]
$$

3.1.4. The translation automorphism $\sigma: E^{g} \rightarrow E^{g}$

As in [CKS19b, §2.4], we define

$$
d\left(n_{1}, \ldots, n_{g}\right):=\operatorname{det}\left(\begin{array}{ccccc}
n_{1} & -1 & & & \\
-1 & n_{2} & -1 & & \\
& -1 & \ddots & \ddots & \\
& & \ddots & n_{g-1} & -1 \\
& & & -1 & n_{g}
\end{array}\right) .
$$

Using this notation, we define $k_{i}$ and $l_{i}$ for $i=0, \ldots, g+1$ by

$$
k_{i}:=d\left(n_{i+1}, \ldots, n_{g}\right) \quad \text { and } \quad l_{i}:=d\left(n_{i-1}, \ldots, n_{1}\right),
$$


with conventions $k_{g}=1, k_{g+1}=0, l_{0}=0$ and $l_{1}=1$. By [CKS19b, Proposition 2.6], we have the formulas

$$
\frac{k_{i-1}}{k_{i}}=\left[n_{i}, \ldots, n_{g}\right] \quad \text { and } \quad \frac{l_{i+1}}{l_{i}}=\left[n_{i}, \ldots, n_{1}\right]
$$

for $i=1, \ldots, g$ and

$$
k_{0}=l_{g+1}=d\left(n_{1}, \ldots, n_{g}\right)=n, \quad k_{1}=k, \quad l_{g}=k^{\prime},
$$

where $n>k^{\prime} \geq 1$ and $k k^{\prime} \equiv 1(\bmod n)$. In [CKS19b, §2.4.1], we also observed

$$
k_{i} n_{i}=k_{i-1}+k_{i+1} \quad \text { and } \quad l_{i} n_{i}=l_{i-1}+l_{i+1}
$$

for $i=1, \ldots, g$. We will use these in section 7 .

For $i=1, \ldots, g$, we define $\tau_{i}:=\left(k_{i}+l_{i}-n\right) \tau$ and define the automorphism $\sigma: \mathbb{C}^{g} \rightarrow \mathbb{C}^{g}$ by

$$
\sigma\left(z_{1}, \ldots, z_{g}\right):=\left(z_{1}+\tau_{1}, \ldots, z_{g}+\tau_{g}\right) .
$$

Because $\left(\mathbb{C}^{g},+\right)$ is an abelian group, all translation automorphisms of it commute with one another. In particular, $\sigma$ commutes with the translation action of $\Lambda^{g}$ on $\mathbb{C}^{g}$, and therefore induces an automorphism of $E^{g}=\mathbb{C}^{g} / \Lambda^{g}$ that we will also denote by $\sigma$.

3.1.5. The group $\Sigma_{n / k} \subseteq \operatorname{Aut}\left(E^{g}\right)$

Let $\Sigma_{n / k}:=\left\langle s_{i} \mid n_{i}=2\right\rangle$, where $s_{i}: E^{g} \rightarrow E^{g}$ is the automorphism

$$
s_{i}\left(z_{1}, \ldots, z_{g}\right):=\left(z_{1}, \ldots, z_{i-1}, z_{i-1}-z_{i}+z_{i+1}, z_{i+1}, \ldots, z_{g}\right),
$$

with the convention that $z_{0}=0$ and $z_{g+1}=0$.

3.1.6. The invertible sheaf $\mathcal{L}_{n / k}$ and the characteristic variety $X_{n / k}$

Following Odesskii and Feigin [OF89, §3.3], we define an invertible sheaf $\mathcal{L}_{n / k}$ on $E^{g}$ as follows. ${ }^{6}$ Let $\mathcal{L}=\mathcal{O}_{E}((0))$ be the degree-one invertible $\mathcal{O}_{E}$-module corresponding to the divisor $(0)$, and define

$$
\mathcal{L}_{n / k}:=\left(\mathcal{L}^{n_{1}} \otimes \cdots \otimes \mathcal{L}^{n_{g}}\right) \otimes\left(\bigotimes_{j=1}^{g-1} \operatorname{pr}_{j, j+1}^{*} \mathcal{P}\right),
$$

where $\mathcal{P}$ is the Poincaré bundle $\left(\mathcal{L}^{-1} \otimes \mathcal{L}^{-1}\right)(\Delta)$ on $E \times E, \mathrm{pr}_{j, j+1}: E^{g} \rightarrow E \times E$ is the projection $\left(z_{1}, \ldots, z_{g}\right) \mapsto\left(z_{j}, z_{j+1}\right)$ and $\Delta=\{(z, z) \mid z \in E\}$.

Thus $\mathcal{L}_{n / k}=\mathcal{O}_{E g}\left(D_{n / k}\right)$, where

$$
D_{n / k}:=\sum_{i=1}^{g} E^{i-1} \times D_{i} \times E^{g-i}+\sum_{j=1}^{g-1} \Delta_{j, j+1},
$$

$\Delta_{j, j+1}=\operatorname{pr}_{j, j+1}^{*} \Delta$ and $D_{i}:=\left(n_{i}-2+\delta_{i, 1}+\delta_{i, g}\right)(0)$. If $g \geq 2$, then

$$
D_{i}= \begin{cases}\left(n_{i}-1\right)(0) & \text { if } i \in\{1, g\} \\ \left(n_{i}-2\right)(0) & \text { if } 2 \leq i \leq g-1\end{cases}
$$

\footnotetext{
${ }^{6}$ In [CKS19b, §3.1.3] we relate this definition to Odesskii and Feigin’s original definition.
} 
A standard divisor of type $\left(n_{1}, \ldots, n_{g}\right)$ is a divisor of the form

$$
D_{\mathfrak{D}_{i}, z_{j}}:=\sum_{i=1}^{g} E^{i-1} \times \mathfrak{D}_{i} \times E^{g-i}+\sum_{i=1}^{g-1} \Delta_{j, j+1}^{z_{j}},
$$

where $\mathfrak{D}_{i}(1 \leq i \leq g)$ are effective divisors on $E$ of respective degrees $\left(n_{i}-2+\delta_{i, 1}+\delta_{i, g}\right), z_{j} \in E$ $(1 \leq j \leq g-1)$ are points, $\Delta_{j, j+1}^{z_{j}}=\operatorname{pr}_{j, j+1}^{*} \Delta^{z_{j}}$ and

$$
\Delta^{z_{j}}:=\left\{\left(z, z+z_{j}\right) \mid z \in E\right\} \subseteq E^{2} .
$$

Proposition 3.1 ([CKS19b, $\S \S 3$ and 4]). If $D$ is a standard divisor of type $\left(n_{1}, \ldots, n_{g}\right)$ with $n_{i} \geq 2$ for all $i$, then $\mathcal{O}_{E^{g}}(D)$ has the following properties:

(1) It is base-point free or, equivalently, generated by its global sections.

(2) It is ample.

(3) It is very ample if and only if $n_{i} \geq 3$ for all $i$.

(4) $\operatorname{dim}_{\mathbb{C}}\left(H^{0}\left(E^{g}, \mathcal{O}_{E^{g}}(D)\right)\right)=n$.

(5) $H^{q}\left(E^{g}, \mathcal{O}_{E^{g}}(D)\right)=0$ for all $q \geq 1$.

In particular, $\mathcal{L}_{n / k}=\mathcal{O}_{E^{g}}\left(D_{n / k}\right)$ satisfies these properties.

Since $\mathcal{L}_{n / k}$ is base-point free, the complete linear system $\left|D_{n / k}\right|$ determines a morphism

$$
\Phi_{n / k}: E^{g} \longrightarrow \mathbb{P}^{n-1}=\mathbb{P}\left(H^{0}\left(E^{g}, \mathcal{L}_{n / k}\right)^{*}\right)
$$

The characteristic variety for $Q_{n, k}(E, \tau)$ is $X_{n / k}:=$ the image of $\Phi_{n / k}$.

\subsubsection{Special cases}

The following examples illustrate some of the possibilities:

(1) If $n \geq 3$ and $k=1$, then $X_{n / k}=E, \sigma$ is translation by $(2-n) \tau$ and $\mathcal{L}_{n / k}$ is an invertible $\mathcal{O}_{E}$-module of degree $n$.

(2) If $\left[n_{1}, \ldots, n_{g}\right]=[2, \ldots, 2]$, then $g=n-1=k, \Phi_{n / k}: E^{g} \rightarrow \mathbb{P}^{n-1}$ is surjective ([CKS19b, $\S 4.6 .2]), Q_{n, n-1}(E, \tau)$ is a polynomial ring on $n$ variables and $\operatorname{QGr}\left(Q_{n, n-1}(E, \tau)\right)=\mathrm{Qcoh}\left(\mathbb{P}^{n-1}\right)$ (see the footnote in $\S 1.2$ ).

(3) If $n_{i} \geq 3$ for all $i$, then $X_{n / k} \cong E^{g}$ is an isomorphism, and conversely [CKS19b, §4.6.1].

(4) If $f_{0}=f_{1}=1, f_{i+1}=f_{i}+f_{i-1}$ and $(n, k)=\left(f_{2 g+1}, f_{2 g-1}\right)$, then $\frac{n}{k}=[3, \ldots, 3]$ and $X_{n / k} \cong E^{g}$.

(5) If $m \geq 3$ and $\left[n_{1}, \ldots, n_{g}\right]$ is either $[m, 2, \ldots, 2,2]$ or $[2,2, \ldots, 2, m]$, then $X_{n / k} \cong S^{g} E$, and conversely [CKS19b, Corollary 4.24].

(6) $X_{(2 k+1) / k} \cong S^{g} E$, since $\frac{2 k+1}{k}=[3,2, \ldots, 2]$ (see $\$ 1.4 .3$ for the significance of this case).

(7) $X_{n^{2} / n-1} \cong S^{n-1} E$ when $n \geq 2$, because $\frac{n^{2}}{n-1}=[n+2,2, \ldots, 2]=\left[n+2,2^{n-2}\right]$ (see Proposition 7.1(1)). The algebras $Q_{n^{2}, n-1}(E, \tau)$ were studied by Cherednik in [Che86]. They are, in a sense, homogenised elliptic versions of the quantised enveloping algebras $U_{q}\left(\mathfrak{s l}_{n}\right)$. Or conversely, the $U_{q}\left(\mathfrak{s l}_{n}\right)$ are 'degenerations' of $Q_{n^{2}, n-1}(E, \tau)$. A detailed examination of this degeneration process for $n=2$ is carried out in [CSW18].

\subsection{Twisted homogeneous coordinate rings related to $Q_{n, k}(E, \tau)$}

Let

$$
\mathcal{L}_{n / k}^{\prime}:=\left.\mathcal{O}_{\mathbb{P}^{n-1}}(1)\right|_{X_{n / k}}
$$

In Corollary 3.6 we obtain a graded $\mathbb{C}$-algebra homomorphism $Q_{n / k}(E, \tau) \rightarrow B\left(X_{n / k}, \sigma^{\prime}, \mathcal{L}_{n / k}^{\prime}\right)$ that is an isomorphism in degree one. 
Theorem 3.2. Let $f: E^{g} \rightarrow X_{n / k}$ be the corestriction of the morphism $\Phi_{n / k}$.

(1) The map $f: E^{g} \rightarrow X_{n / k}$ is a quotient morphism for the action of $\Sigma_{n / k}$ on $E^{g}$.

(2) There is a unique automorphism $\sigma^{\prime}$ of $X_{n / k}$ such that $\Phi_{n / k} \circ \sigma=\sigma^{\prime} \circ \Phi_{n / k}$.

(3) There is a morphism of triples $(f, u):\left(E^{g}, \sigma, \mathcal{L}_{n / k}\right) \rightarrow\left(X_{n / k}, \sigma^{\prime}, \mathcal{L}_{n / k}^{\prime}\right)$.

(4) There is a homomorphism of graded algebras $B\left(X_{n / k}, \sigma^{\prime}, \mathcal{L}_{n / k}^{\prime}\right) \longrightarrow B\left(E^{g}, \sigma, \mathcal{L}_{n / k}\right)$.

(5) The group $\Sigma_{n / k}$ acts as automorphisms of $B\left(E^{g}, \sigma, \mathcal{L}_{n / k}\right)$, and the corestriction of the homomorphism in (4) is an isomorphism

$$
B\left(X_{n / k}, \sigma^{\prime}, \mathcal{L}_{n / k}^{\prime}\right) \stackrel{\sim}{\longrightarrow} B\left(E^{g}, \sigma, \mathcal{L}_{n / k}\right)^{\Sigma_{n / k}} .
$$

(6) Every ample invertible sheaf on $X_{n / k}$, in particular $\mathcal{L}_{n / k}^{\prime}$, is $\sigma^{\prime}$-ample.

(7) There are isomorphisms $\mathcal{L}_{n / k}^{\prime} \cong\left(f_{*} \mathcal{L}_{n / k}\right)^{\Sigma_{n / k}}$ and $\mathcal{L}_{n / k} \cong f^{*} \mathcal{L}_{n / k}^{\prime}$.

Proof.

(1) See [CKS19b, Corollary 4.19].

(2) This follows from (1) and [CKS19b, Proposition 2.10].

(3) This follows from the definitions of $X_{n / k}$ and $\mathcal{L}_{n / k}^{\prime}$. More explicitly, if $\iota: X_{n / k} \rightarrow$ $\mathbb{P}\left(H^{0}\left(E^{g}, \mathcal{L}_{n / k}\right)^{*}\right)$ is the inclusion morphism, then $\Phi_{n / k}=\iota \circ f$ and $\mathcal{L}_{n / k} \cong \Phi_{n / k}^{*} \mathcal{O}_{\mathbb{P} n-1}(1)=$ $f^{*} \iota^{*} \mathcal{O}_{\mathbb{P} n-1}(1)=f^{*} \mathcal{L}_{n / k}^{\prime}$, so we take $u$ to be the canonical isomorphism $f^{*} \mathcal{L}_{n / k}^{\prime} \rightarrow \mathcal{L}_{n / k}$.

(4) This follows from (3) and Proposition 2.1.

(5) By [CKS19b, Proposition 4.11], $\mathcal{L}_{n / k}$ is a $\Sigma_{n / k}$-equivariant sheaf on $E^{g}-$ that is, there are isomorphisms $t_{\gamma}: \mathcal{L}_{n / k} \rightarrow \gamma^{*} \mathcal{L}_{n / k}, \gamma \in \Sigma_{n / k}$, such that $t_{\alpha \beta}=\beta^{*}\left(t_{\alpha}\right) \circ t_{\beta}$ for all $\alpha, \beta \in \Sigma_{n / k}$. Since the action of $\sigma$ commutes with that of $\Sigma_{n / k}$, each pair $\left(\gamma, t_{\gamma}^{-1}\right)$ is an automorphism of the triple $\left(E^{g}, \sigma, \mathcal{L}_{n / k}\right)$ and therefore induces (by functoriality) a right action of $\Sigma_{n / k}$ as automorphisms of $B\left(E^{g}, \sigma, \mathcal{L}_{n / k}\right){ }^{7}$

For brevity we write $\mathcal{L}=\mathcal{L}_{n / k}$ and $\mathcal{L}^{\prime}=\mathcal{L}_{n / k}^{\prime}$.

Comparing the degree- $(m+1)$ components in (3.6), we must show that the natural map

$$
H^{0}\left(E^{g} / \Sigma_{n / k}, \mathcal{L}^{\prime} \otimes \sigma^{*} \mathcal{L}^{\prime} \otimes \cdots \otimes\left(\sigma^{\prime m}\right)^{*} \mathcal{L}^{\prime}\right) \longrightarrow H^{0}\left(E^{g}, \mathcal{L} \otimes \sigma^{*} \mathcal{L} \otimes \cdots \otimes\left(\sigma^{m}\right)^{*} \mathcal{L}\right)^{\Sigma_{n / k}}
$$

is an isomorphism for all $m \geq 0$.

For simplicity we assume $m=2$; all other cases are essentially the same. We will show that the natural map

$$
H^{0}\left(E^{g} / \Sigma_{n / k}, \mathcal{L}^{\prime} \otimes \sigma^{*} \mathcal{L}^{\prime}\right) \longrightarrow H^{0}\left(E^{g}, \mathcal{L} \otimes \sigma^{*} \mathcal{L}\right)^{\Sigma_{n / k}}
$$

is an isomorphism. Since $\sigma^{\prime} \circ f=f \circ \sigma, f^{*} \circ\left(\sigma^{\prime i}\right)^{*}=\left(\sigma^{i}\right)^{*} \circ f^{*}$ for all $i$; hence, since $f^{*}$ commutes with $\otimes$ and $\mathcal{L} \cong f^{*} \mathcal{L}^{\prime}$,

$$
\mathcal{L} \otimes \sigma^{*} \mathcal{L} \cong f^{*}\left(\mathcal{L}^{\prime} \otimes \sigma^{*} \mathcal{L}^{\prime}\right)
$$

Therefore, by the Projection Formula, $f_{*}\left(\mathcal{L} \otimes \sigma^{*} \mathcal{L}\right) \cong f_{*} \mathcal{O}_{E^{g}} \otimes \mathcal{L}^{\prime} \otimes \sigma^{* *} \mathcal{L}^{\prime}$. Since the action of $\Sigma_{n / k}$ on $\mathcal{L}^{\prime}$ and $\sigma^{* *} \mathcal{L}^{\prime}$ is trivial, it follows that

$$
\left(f_{*}\left(\mathcal{L} \otimes \sigma^{*} \mathcal{L}\right)\right)^{\Sigma_{n / k}} \cong\left(f_{*} \mathcal{O}_{E g}\right)^{\Sigma_{n / k}} \otimes \mathcal{L}^{\prime} \otimes \sigma^{*} \mathcal{L}^{\prime}=\mathcal{L}^{\prime} \otimes \sigma^{\prime *} \mathcal{L}^{\prime}
$$

Hence

$$
\begin{aligned}
H^{0}\left(E^{g}, \mathcal{L} \otimes \sigma^{*} \mathcal{L}\right)^{\Sigma_{n / k}} & \cong H^{0}\left(E^{g} / \Sigma_{n / k}, f_{*}\left(\mathcal{L} \otimes \sigma^{*} \mathcal{L}\right)\right)^{\Sigma_{n / k}} \\
& \cong H^{0}\left(E^{g} / \Sigma_{n / k},\left(f_{*}\left(\mathcal{L} \otimes \sigma^{*} \mathcal{L}\right)\right)^{\Sigma_{n / k}}\right) \\
& \cong H^{0}\left(E^{g} / \Sigma_{n / k}, \mathcal{L}^{\prime} \otimes \sigma^{\prime *} \mathcal{L}^{\prime}\right)
\end{aligned}
$$

\footnotetext{
${ }^{7}$ The triple $\left(E^{g}, \sigma, \mathcal{L}_{n / k}\right)$ is a $\Sigma_{n / k}$-triple in the terminology of [ST94, p. 27]. In [ST94] the group acts freely, but the terminology extends to the present situation.
} 
Thus the map in (3.7) is an isomorphism in degree two.

(6) This is an immediate consequence of (1) and Corollary 2.7.

(7) See [CKS19b, Remark 4.10, Proposition 4.5 and the remarks at the beginning of §4].

Remark 3.3. Part (5) of Theorem 3.2 is essentially [ST94, Proposition 2.5]. The difference is that in the latter, the action of the group $\left(\Sigma_{n / k}\right)$ is free. An examination of the proof, however, reveals that the only consequence of freeness needed there is the fact that

$$
\mathcal{L}_{n / k} \cong f^{*} \mathcal{L}_{n / k}^{\prime} \cong f^{*}\left(\left(f_{*} \mathcal{L}_{n / k}\right)^{\Sigma_{n / k}}\right)
$$

This, in turn, is a consequence of [CKS19b, Proposition 4.5].

By Proposition 2.2, $B\left(X, \sigma^{-1}, \mathcal{L}\right)^{\mathrm{op}} \cong B(X, \sigma, \mathcal{L})$. In the context of elliptic algebras, more is true.

Theorem 3.4. $B\left(X_{n / k}, \sigma^{\prime}, \mathcal{L}_{n / k}^{\prime}\right) \cong B\left(X_{n / k},\left(\sigma^{\prime}\right)^{-1}, \mathcal{L}_{n / k}^{\prime}\right)$.

Proof. Let [-1] : $E^{g} \rightarrow E^{g}$ be the automorphism z $\mapsto-z$ and let $\mu: X_{n / k} \rightarrow X_{n / k}$ be the automorphism that is the descent of $[-1]$. Since $\sigma$ is a translation automorphism, $\sigma^{-1}=[-1]^{-1} \circ \sigma \circ[-1]$; hence $\left(\sigma^{\prime}\right)^{-1}=\mu^{-1} \sigma^{\prime} \mu$. We will complete the proof by applying Proposition 2.3 to $\left(X_{n / k}, \sigma^{\prime}, \mathcal{L}_{n / k}^{\prime}\right)$ after showing that $\mu^{*} \mathcal{L}_{n / k}^{\prime} \cong \mathcal{L}_{n / k}^{\prime}$.

The action of $\mathbb{Z}_{2}=\{\mathrm{id},[-1]\}$ on $E^{g}$ preserves the effective divisor $D_{n / k}$ as a subscheme. By [CKS19b, Lemma 4.8], this gives a $\mathbb{Z}_{2}$-equivariant structure on $\mathcal{L}_{n / k}$. Setting $V=H^{0}\left(E^{g}, \mathcal{L}_{n / k}\right)$, we now have a $\mathbb{Z}_{2}$-action on $V$ inducing one on $\mathbb{P}\left(V^{*}\right)$ together with a compatible $\mathbb{Z}_{2}$-equivariant structure on the twisting sheaf $\mathcal{O}_{\mathbb{P}\left(V^{*}\right)}(1)$ (see, e.g., [MFK94, Proposition 1.7]). The morphism $\Phi_{n / k}: E^{g} \rightarrow \mathbb{P}\left(V^{*}\right)$ is $\mathbb{Z}_{2}$-equivariant and the generator of $\mathbb{Z}_{2}$ acts on $X_{n / k}$ as $\mu$. The equivariant structure on $\mathcal{O}_{\mathbb{P}\left(V^{*}\right)}(1)$ restricts to one on

$$
\left.\mathcal{O}_{\mathbb{P}\left(V^{*}\right)}(1)\right|_{X_{n / k}} \cong \mathcal{L}_{n / k}^{\prime},
$$

whence the desired isomorphism $\mu^{*} \mathcal{L}_{n / k}^{\prime} \cong \mathcal{L}_{n / k}^{\prime}$, which completes the proof.

Let $\Theta_{n / k}(\Lambda)$ be the space of theta functions in $g$ variables defined in [CKS19b, $\$ 2.7$ and 5.2], and let $\left\{w_{\alpha}(z) \mid \alpha \in \mathbb{Z}_{n}\right\}$ be the basis for $\Theta_{n / k}(\Lambda)$ in [CKS19b, §5.1.1]. We make the identifications $Q_{n, k}(E, \tau)_{1}=H^{0}\left(E^{g}, \mathcal{L}_{n / k}\right)=\Theta_{n / k}(\Lambda)$ described in [CKS19b, §5.3]. The identifications are such that $x_{\alpha}=w_{\alpha}(\mathrm{z})$, and the morphism $\Phi_{n / k}: E^{g} \rightarrow \mathbb{P}\left(H^{0}\left(E^{g}, \mathcal{L}_{n / k}\right)^{*}\right)$ is given by $\Phi_{n / k}(\mathrm{z})=\left(w_{0}(\mathrm{z}), \ldots, w_{n-1}(\mathrm{z})\right)$.

Proposition 3.5 ([CKS19b, Corollary 5.9]). The quadratic relations for $Q_{n, k}(E, \tau)$ vanish on the graph of the automorphism $\sigma^{\prime}: X_{n / k} \rightarrow X_{n / k}$.

Corollary 3.6. There are $\mathbb{C}$-algebra homomorphisms

$$
Q_{n, k}(E, \tau) \stackrel{\Psi_{n / k}}{\longrightarrow} B\left(X_{n / k}, \sigma^{\prime}, \mathcal{L}_{n / k}^{\prime}\right) \longrightarrow B\left(E^{g}, \sigma, \mathcal{L}_{n / k}\right)
$$

that are isomorphisms in degree one.

Proof. Let $\varphi: Q_{n, k}(E, \tau)_{1} \longrightarrow \Theta_{n / k}(\Lambda)=H^{0}\left(E^{g}, \mathcal{L}_{n / k}\right)=H^{0}\left(X_{n / k}, \mathcal{L}_{n / k}^{\prime}\right)=B\left(X_{n / k}, \sigma^{\prime}, \mathcal{L}_{n / k}^{\prime}\right)_{1}$ be the vector-space isomorphism defined by $\varphi\left(x_{\alpha}\right)=w_{\alpha}(\mathrm{z})$. By Proposition 2.9, $\varphi$ extends to the desired algebra homomorphism if the degree-two relations for $Q_{n, k}(E, \tau)$ vanish on

$$
\left\{\left(\Phi_{n / k}(\mathrm{z}), \Phi_{n / k}(\sigma(\mathrm{z}))\right) \mid \mathrm{z} \in E^{g}\right\} .
$$

They do, by Proposition 3.5.

Corollary 3.7. Assume $k \neq n-1$. Suppose the homomorphism $Q_{n, k}(E, \tau) \rightarrow B\left(X_{n / k}, \sigma^{\prime}, \mathcal{L}_{n / k}^{\prime}\right)$ is surjective. If $Q_{n, k}(E, \tau)$ is a finitely generated module over its centre, then $\tau$ has finite order. 
Proof. Since the homomorphism is surjective, $B\left(X_{n / k}, \sigma^{\prime}, \mathcal{L}_{n / k}^{\prime}\right)$ is also finite over its centre. Let $K$ denote the field of rational functions of $X_{n / k}$, and let $K\left[t^{ \pm 1} ; \sigma^{\prime}\right]$ denote the skew Laurent polynomial extension associated to the automorphism $\sigma^{\prime}$ of $K$. By [ST94, Proposition 2.1], $K\left[t^{ \pm 1} ; \sigma^{\prime}\right]$ is a localisation of $B\left(X_{n / k}, \sigma^{\prime}, \mathcal{L}_{n / k}^{\prime}\right)$, so it is also finite over its centre. It is well known, and easy to show, that the fact that $K\left[t^{ \pm 1} ; \sigma^{\prime}\right]$ is finite over its centre implies that $\sigma^{\prime}$ has finite order as an automorphism of $K$ and hence as an automorphism of $X_{n / k}$. If that order is $b$, then $\sigma^{b}(p)$ and $p$ have the same image in $X_{n / k}$ for all $p \in E^{g}$. Thus, if $p \in E^{g}$, then $\sigma^{b}(p)=\gamma \cdot p$ for some $\gamma \in \Sigma_{n / k}$. If $a$ denotes the size of $\Sigma_{n / k}$, then $\sigma^{a b}(p)=p$ for all $p \in E^{g}$.

But $\sigma$ is translation by $(\mathrm{k}+\mathrm{I}-\mathrm{n}) \tau$, so in particular, $\left(k_{1}+l_{1}-n\right) \tau$ has finite order. But $k_{1}=k$ and $l_{1}=1$, so $k_{1}+l_{1}-n \neq 0$. Hence $\tau$ has finite order.

It is stated at [OF89, p. 209, Remark 1] and [Ode02, p. 1143] that $Q_{n, n-1}(E, \tau)$ is a polynomial ring for all $E$ and $\tau$. We proved this in [CKS18, Proposition 5.5]. The proof is a direct calculation and also uses the fact that the space of relations for $Q_{n, n-1}(E, \tau)$ has dimension $\left(\begin{array}{l}n \\ 2\end{array}\right)$. The direct-calculation part has an alternative proof using the twisted homogeneous coordinate ring.

Corollary 3.8. $Q_{n, n-1}(E, \tau)$ is a polynomial ring on $n$ variables.

Proof. An induction argument shows that $\frac{n}{n-1}=[2, \ldots, 2]$, where the number of $2 \mathrm{~s}$ is $n-1$. Thus $g=$ $n-1$. Hence $\Sigma_{n /(n-1)}=\Sigma_{g+1}$ and $X_{n /(n-1)} \cong \mathbb{P}^{n-1}$. Corollary 3.6 therefore provides a homomorphism $Q_{n, n-1}(E, \tau) \rightarrow B\left(\mathbb{P}^{n-1}, \sigma^{\prime}, \mathcal{O}_{\mathbb{P}^{n-1}}(1)\right)$ that is surjective in degree one.

The numbers $k_{i}$ and $l_{i}$ defined in $\$ 3.1 .4$ are $\left(k_{1}, \ldots, k_{n-1}\right)=(n-1, \ldots, 2,1)$ and $\left(l_{1}, \ldots, l_{n-1}\right)=$ $(1,2, \ldots, n-1)$, so $k_{i}+l_{i}-n=0$ for all $i=1, \ldots, n-1$. Hence $\sigma$ and $\sigma^{\prime}$ are the identity morphisms. In particular, $B\left(X_{n / k}, \sigma^{\prime}, \mathcal{L}_{n / k}^{\prime}\right)=B\left(\mathbb{P}^{n-1}, \mathrm{id}, \mathcal{O}(1)\right)=\bigoplus_{i \geq 0} H^{0}\left(\mathbb{P}^{n-1}, \mathcal{O}(i)\right)$. This is a polynomial ring on $n$ variables, so the homomorphism $Q_{n, n-1}(E, \tau) \rightarrow B\left(\mathbb{P}^{n-1}, \sigma^{\prime}, \mathcal{O}_{\mathbb{P} n-1}(1)\right)$ is surjective. It is also injective, because the quadratic relations for both $Q_{n, n-1}(E, \tau)$ and $B\left(\mathbb{P}^{n-1}\right.$, id, $\left.\mathcal{O}(1)\right)$ span vector spaces of dimension $\left(\begin{array}{l}n \\ 2\end{array}\right)$.

Proposition 3.9. There is a commutative diagram

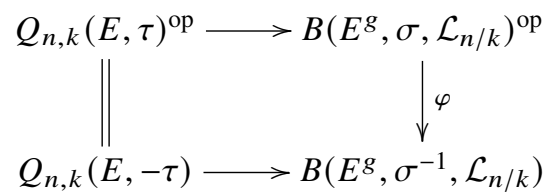

in which the horizontal arrows are given by the composition in (3.8) and $\varphi$ is the isomorphism in Proposition 2.2.

Proof. By [CKS18, Proposition 3.22], $Q_{n, k}(E, \tau)^{\mathrm{op}}=Q_{n, k}(E,-\tau)$, because the space of relations for $Q_{n, k}(E, \tau)^{\mathrm{op}}$ is the same subspace of $V^{\otimes 2}$ as the space of relations for $Q_{n, k}(E,-\tau)$. Since $Q_{n, k}(E, \tau)$ is generated by its degree-one component, to show that the diagram commutes we need only check that it commutes in degree one. This is true because $\varphi$ is the identity map in degree one, and so are the horizontal maps.

\subsubsection{Remark}

The homomorphisms in Corollary 3.6 do not give all homomorphisms to twisted homogeneous coordinate rings. For example, there are four surjective homomorphisms from $Q_{4,1}(E, \tau)$ to the polynomial ring in one variable, corresponding to the four isolated point modules. If we present $Q_{4,1}(E, \tau)$ as Sklyanin does, then those homomorphisms are obtained by quotienting out three of the four generators for the algebra [LS93, Proposition 5.2]. Similarly, the remarks at the end of [CKS19b, §5.5] exhibit four surjective homomorphisms from $Q_{8,3}(E, \tau)$ to the polynomial ring on two variables. 


\section{Semistable and locally free $\mathcal{O}_{E}$-modules}

We need some standard results on semistable locally free sheaves on a smooth projective curve $C$. Loring Tu's paper [Tu93] is a good source for these results when $C$ is the elliptic curve $E$.

In this and subsequent sections, a locally free sheaf always means a locally free coherent sheaf, that is, of finite type.

\subsection{Semistable $\mathcal{O}_{C}$-modules}

Let $C$ be a smooth projective curve.

The slope of a nonzero locally free $\mathcal{O}_{C}$-module $\mathcal{F}$ is the number

$$
\mu(\mathcal{F}):=\frac{\operatorname{deg}(\mathcal{F})}{\operatorname{rank}(\mathcal{F})} .
$$

We say $\mathcal{F}$ is

○ semistable if $\mu\left(\mathcal{F}^{\prime}\right) \leq \mu(\mathcal{F})$ for all nonzero $\mathcal{F}^{\prime} \subseteq \mathcal{F}$ and

○ stable if $\mu\left(\mathcal{F}^{\prime}\right)<\mu(\mathcal{F})$ for all nonzero $\mathcal{F}^{\prime} \subsetneq \mathcal{F}$.

Lemma 4.1. If $0 \rightarrow \mathcal{F}^{\prime} \rightarrow \mathcal{F} \rightarrow \mathcal{F}^{\prime \prime} \rightarrow 0$ is an exact sequence of nonzero locally free $\mathcal{O}_{C}$-modules, then either

(1) $\mu\left(\mathcal{F}^{\prime}\right)<\mu(\mathcal{F})<\mu\left(\mathcal{F}^{\prime \prime}\right)$ or

(2) $\mu\left(\mathcal{F}^{\prime}\right)=\mu(\mathcal{F})=\mu\left(\mathcal{F}^{\prime \prime}\right)$ or

(3) $\mu\left(\mathcal{F}^{\prime}\right)>\mu(\mathcal{F})>\mu\left(\mathcal{F}^{\prime \prime}\right)$.

In particular, $\min \left\{\mu\left(\mathcal{F}^{\prime}\right), \mu\left(\mathcal{F}^{\prime \prime}\right)\right\} \leq \mu(\mathcal{F}) \leq \max \left\{\mu\left(\mathcal{F}^{\prime}\right), \mu\left(\mathcal{F}^{\prime \prime}\right)\right\}$.

(4) If $\mathcal{F}$ is semistable, then (1) or (2) holds.

(5) If $\mathcal{F}$ is stable, then (1) holds.

Lemma 4.2. All direct summands of a semistable $\mathcal{O}_{C}$-module $\mathcal{F}$ have the same slope as $\mathcal{F}$.

Lemma 4.3. If $\mathcal{A}$ and $\mathcal{B}$ are semistable and $0 \rightarrow \mathcal{A} \rightarrow \oplus \mathcal{V}_{i} \rightarrow \mathcal{B} \rightarrow 0$ is an exact sequence of locally free $\mathcal{O}_{C}$-modules, then $\mu\left(\mathcal{V}_{i}\right) \geq \min \{\mu(\mathcal{A}), \mu(\mathcal{B})\}$ for all $i$.

Proof. Fix $j$ and let $\pi: \oplus \mathcal{V}_{i} \rightarrow \mathcal{V}_{j}$ be the projection. Let $\mathcal{U}_{j}$ be the image of $\mathcal{A}$ in $\mathcal{V}_{j}$. There is a commutative diagram

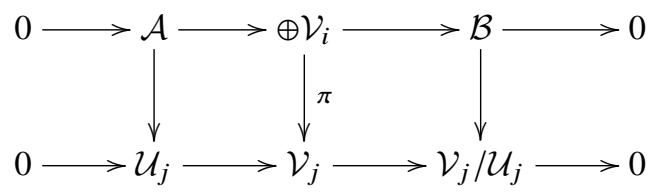

with exact rows. The leftmost vertical arrow is an epimorphism by definition, so by the Snake Lemma the rightmost vertical arrow is also an epimorphism. Since $\mathcal{U}_{j}$ and $\mathcal{V}_{j} / \mathcal{U}_{j}$ are quotients of the semistable sheaves $\mathcal{A}$ and $\mathcal{B}$, respectively, Lemma 4 .1 tells us that $\mu\left(\mathcal{U}_{j}\right) \geq \mu(\mathcal{A})$ and $\mu\left(\mathcal{V}_{j} / \mathcal{U}_{j}\right) \geq \mu(\mathcal{B})$. Lemma 4.1 also tells us that $\mu\left(\mathcal{V}_{j}\right) \geq \min \left\{\mu\left(\mathcal{U}_{j}\right), \mu\left(\mathcal{V}_{j} / \mathcal{U}_{j}\right)\right\}$. The result follows.

Lemma 4.4. If $\mathcal{U}$ and $\mathcal{V}$ are nonzero locally free $\mathcal{O}_{C}$-modules, then

$$
\mu(\mathcal{U} \otimes \mathcal{V})=\mu(\mathcal{U})+\mu(\mathcal{V})
$$

Proof. This follows from the fact that $\operatorname{deg}(\mathcal{U} \otimes \mathcal{V})=\operatorname{deg}(\mathcal{U}) \operatorname{rank}(\mathcal{V})+\operatorname{deg}(\mathcal{V}) \operatorname{rank}(\mathcal{U})$. 
Lemma 4.5 ([Tu93, Appendix A]). Let $\mathcal{F}$ be a locally free $\mathcal{O}_{E}$-module. If $\mathcal{F}$ is indecomposable, then it is semistable, and it is stable if and only if its degree and rank are coprime. ${ }^{8}$

Lemma 4.6 ([Mar81, Theorem 2.5]). Assume char $(\mathbb{k})=0$. If $\mathcal{U}$ and $\mathcal{V}$ are semistable locally free $\mathcal{O}_{C^{-}}$ modules, so is $\mathcal{U} \otimes \mathcal{V}$.

Proposition 4.7. If $\operatorname{char}(\mathbb{k})=0$, then the tensor product of two indecomposable locally free $\mathcal{O}_{E}$-modules is a direct sum of indecomposable sheaves with equal slopes.

Proof. Combine Lemmas 4.2, 4.5 and 4.6.

Lemma 4.8. Let $\mathcal{F}$ be a semistable locally free $\mathcal{O}_{E}$-module.

(1) If $\operatorname{deg}(\mathcal{F})>0$, then $\operatorname{dim} H^{0}(\mathcal{F})=\operatorname{deg}(\mathcal{F})$ and $\operatorname{dim} H^{1}(\mathcal{F})=0$.

(2) If $\operatorname{deg}(\mathcal{F})<0$, then $H^{0}(\mathcal{F})=0$

(3) If $\mathcal{F}$ is nonzero and generated by its global sections, then $\operatorname{deg}(\mathcal{F})>0$.

(4) If $\mu(\mathcal{F})>1$, then $\mathcal{F}$ is generated by its global sections.

Proof.

(1) This is [Tu93, Lemma 17].

(2) If $\mathcal{F}$ has a nonzero section, then there is a nonzero map $\mathcal{O}_{E} \rightarrow \mathcal{F}$. The image of this map is isomorphic to $\mathcal{O}_{E}$, so the semistability of $\mathcal{F}$ implies $0=\mu\left(\mathcal{O}_{E}\right) \leq \mu(\mathcal{F})$, whence $0 \leq \operatorname{deg}(\mathcal{F})$.

(3) This is an immediate consequence of (2).

(4) Let $p \in E$. Since $\mathcal{F}(-(p))$ is semistable of slope $\mu(\mathcal{F})-1>0$, its degree is positive, whence $H^{1}(\mathcal{F}(-(p)))=0$ by (1). Therefore, applying $\mathcal{F} \otimes-$ to the sequence $0 \rightarrow \mathcal{O}(-(p)) \rightarrow \mathcal{O}_{E} \rightarrow \mathcal{O}_{p} \rightarrow 0$ and taking cohomology yields an exact sequence $0 \rightarrow H^{0}(\mathcal{F}(-(p))) \rightarrow H^{0}(\mathcal{F}) \rightarrow H^{0}\left(\mathcal{F} \otimes \mathcal{O}_{p}\right) \rightarrow 0$. The fact that $H^{0}(\mathcal{F}) \rightarrow H^{0}\left(\mathcal{F} \otimes \mathcal{O}_{p}\right)$ is onto for all $p$, together with Nakayama's lemma, tells us that $\mathcal{F}$ is generated by its global sections.

\subsection{Surjectivity of multiplication maps}

Theorem 4.9. Let $\mathcal{U}$ and $\mathcal{V}$ be semistable locally free $\mathcal{O}_{E}$-modules generated by their global sections. If

$$
\frac{1}{\mu(\mathcal{U})}+\frac{1}{\mu(\mathcal{V})}<1,
$$

then the canonical map $H^{0}(E, \mathcal{U}) \otimes H^{0}(E, \mathcal{V}) \rightarrow H^{0}(E, \mathcal{U} \otimes \mathcal{V})$ is onto. ${ }^{9}$

Proof. Because they are semistable, $\mathcal{U}$ and $\mathcal{V}$ are direct sums of indecomposable summands of slopes equal to $\mu(\mathcal{U})$ and $\mu(\mathcal{V})$, respectively, so it suffices to prove the result when $\mathcal{U}$ and $\mathcal{V}$ are indecomposable (and therefore semistable); we therefore make this assumption in the rest of the proof. We also assume that $\mathcal{U} \neq 0$ and $\mathcal{V} \neq 0$. By Lemma 4.8(3) and (1), $\operatorname{deg}(\mathcal{U})>0$ and $H^{1}(\mathcal{U})=0$.

Tensoring the exact sequence

$$
0 \longrightarrow \mathcal{K} \stackrel{\varepsilon}{\longrightarrow} H^{0}(E, \mathcal{U}) \otimes \mathcal{O} \longrightarrow \mathcal{U} \longrightarrow 0
$$

with $\mathcal{V}$ and taking cohomology produces an exact sequence

$$
0 \rightarrow H^{0}(E, \mathcal{K} \otimes \mathcal{V}) \rightarrow H^{0}(E, \mathcal{U}) \otimes H^{0}(E, \mathcal{V}) \rightarrow H^{0}(E, \mathcal{U} \otimes \mathcal{V}) \rightarrow H^{1}(E, \mathcal{K} \otimes \mathcal{V})
$$

We will prove the theorem by showing that $H^{1}(E, \mathcal{K} \otimes \mathcal{V})=0$.

By Lemma $4.8(1), H^{1}(E, \mathcal{K} \otimes \mathcal{V})=0$ if $\mathcal{K} \otimes \mathcal{V}$ is semistable of positive degree. That is what we will prove: first we will show $\mathcal{K}$ is indecomposable and hence semistable by Lemma 4.5, which will, by

\footnotetext{
${ }^{8}$ Polishchuk uses the Harder-Narasimhan filtration to show that indecomposability implies semistability [Pol03, Lemma 14.5].

${ }^{9}$ Since $\mathcal{U}$ and $\mathcal{V}$ are generated by their global sections, their degrees are $\geq 0$; hypothesis (4.1) implies that their degrees (or equivalently, their slopes) are, in fact, positive.
} 
Lemma 4.6, imply that $\mathcal{K} \otimes \mathcal{V}$ is semistable; then we will show that its slope, and hence its degree, is positive.

To show that $\mathcal{K}$ is indecomposable, we write it as a direct sum $\mathcal{K}=\mathcal{K}_{1} \oplus \cdots \oplus \mathcal{K}_{n}$ of nonzero indecomposable submodules. Applying the functor $\mathcal{H o m}\left(-, \mathcal{O}_{E}\right)$ to the sequence in (4.2) produces an exact sequence

$$
0 \rightarrow \mathcal{U}^{\vee} \rightarrow H^{0}(E, \mathcal{U})^{*} \otimes \mathcal{O} \rightarrow \mathcal{K}^{\vee} \rightarrow 0
$$

Hence $\mathcal{K}^{\vee}$ is generated by global sections. It follows that every $\mathcal{K}_{i}^{\vee}$ is also generated by its global sections. Since $\mathcal{K}_{i}^{\vee}$ is indecomposable, it is semistable and therefore of positive degree by Lemma 4.8(3). It follows that the kernel of the natural map $H^{0}\left(E, \mathcal{K}_{i}^{\vee}\right) \otimes \mathcal{O} \rightarrow \mathcal{K}_{i}^{\vee}$ is nonzero for all $i$. The kernel of the natural map $H^{0}\left(E, \mathcal{K}^{\vee}\right) \otimes \mathcal{O} \rightarrow \mathcal{K}^{\vee}$ is therefore a direct sum of (at least) $n$ nonzero $\mathcal{O}_{E}$-submodules.

That kernel has another description. By construction, the right-hand map in the sequence in (4.2) induces an isomorphism on global sections, so it follows from the long exact cohomology sequence associated to the sequence in (4.2) that the sequence

$$
0 \longrightarrow H^{1}(E, \mathcal{K}) \stackrel{H^{1}(\varepsilon)}{\longrightarrow} H^{1}\left(E, H^{0}(E, \mathcal{U}) \otimes \mathcal{O}\right) \longrightarrow H^{1}(E, \mathcal{U})=0
$$

is exact. Since $H^{1}(\varepsilon)$ is an isomorphism, $H^{0}\left(\varepsilon^{\vee}\right)$ is also an isomorphism by Serre duality. Thus we obtain a commutative diagram

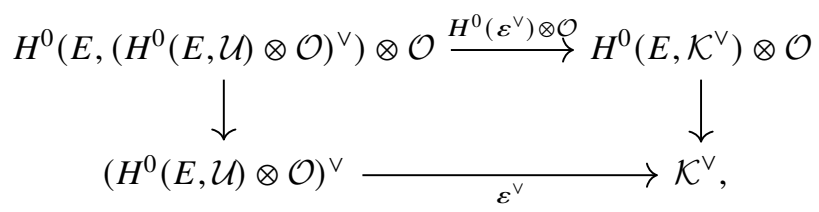

where $H^{0}\left(\varepsilon^{\vee}\right) \otimes \mathcal{O}$ and the left vertical morphism are isomorphisms (since $\left(H^{0}(E, \mathcal{U}) \otimes \mathcal{O}\right)^{\vee}$ is free).

Since $\mathcal{U}$ is indecomposable, so is $\mathcal{U}^{\vee} \cong \operatorname{ker}\left(\varepsilon^{\vee}\right)$. The kernel of the canonical map $H^{0}\left(E, \mathcal{K}^{\vee}\right) \otimes \mathcal{O} \rightarrow$ $\mathcal{K}^{\vee}$ is therefore indecomposable too. However, that kernel is a direct sum of at least $n$ nonzero $\mathcal{O}_{E^{-}}$ submodules, so $n=1-$ that is, $\mathcal{K}$ is indecomposable, as claimed.

To complete the proof we show that $\mu(\mathcal{K} \otimes \mathcal{V})>0$. Recall that

$$
\mu(\mathcal{K} \otimes \mathcal{V})=\mu(\mathcal{K})+\mu(\mathcal{V})
$$

By the definition of $\mathcal{K}$ in (4.2), its degree is $-\operatorname{deg}(\mathcal{U})$ and its rank is

$$
\operatorname{dim}_{\mathbb{K}} H^{0}(E, \mathcal{U})-\operatorname{rank}(\mathcal{U})=\operatorname{deg}(\mathcal{U})-\operatorname{rank}(\mathcal{U})
$$

(the equality follows from Lemma 4.8). The target inequality $\mu(\mathcal{K})+\mu(\mathcal{V})>0$ is thus equivalent to

$$
\mu(\mathcal{V})>-\mu(\mathcal{K})=\frac{\operatorname{deg}(\mathcal{U})}{\operatorname{deg}(\mathcal{U})-\operatorname{rank}(\mathcal{U})}=\frac{1}{1-\frac{1}{\mu(\mathcal{U})}} .
$$

The hypothesis in the statement can be written as

$$
\frac{1}{\mu(\mathcal{V})}<1-\frac{1}{\mu(\mathcal{U})}
$$

and both sides are positive. The proof is complete.

Corollary 4.10. Let $\mathcal{U}$ and $\mathcal{V}$ be locally free $\mathcal{O}_{E}$-modules generated by their global sections and suppose that $\mathcal{U}$ is semistable of slope $>2$. If $0 \rightarrow \mathcal{A} \rightarrow \mathcal{V} \rightarrow \mathcal{B} \rightarrow 0$ is an exact sequence in which $\mathcal{A}$ and $\mathcal{B}$ are 
semistable locally free $\mathcal{O}_{E}$-modules of slope $\geq 2$, then the multiplication map

$$
H^{0}(E, \mathcal{U}) \otimes H^{0}(E, \mathcal{V}) \longrightarrow H^{0}(E, \mathcal{U} \otimes \mathcal{V})
$$

is onto.

Proof. Write $\mathcal{V}=\oplus \mathcal{V}_{i}$ as the sum of its indecomposable summands. By Lemma 4.3, each $\mathcal{V}_{i}$ is locally free, semistable of slope $\geq 2$ and generated by its global sections. Since $\mu(\mathcal{U})^{-1}+\mu\left(\mathcal{V}_{i}\right)^{-1}<1$, the map $H^{0}(\mathcal{U}) \otimes H^{0}\left(\mathcal{V}_{i}\right) \rightarrow H^{0}\left(\mathcal{U} \otimes \mathcal{V}_{i}\right)$ is onto. The conclusion follows from Theorem 4.9.

Corollary 4.11. If $\mathcal{U}$ and $\mathcal{V}$ are semistable locally free $\mathcal{O}_{E}$-modules of slope $>2$, then the multiplication map

$$
H^{0}(E, \mathcal{U}) \otimes H^{0}(E, \mathcal{V}) \longrightarrow H^{0}(E, \mathcal{U} \otimes \mathcal{V})
$$

is onto.

Proof. By Lemma 4.8(4), $\mathcal{U}$ and $\mathcal{V}$ are generated by global sections. By hypothesis, the inequality in (4.1) holds. The result now follows from Theorem 4.9.

\subsection{Remarks}

\subsection{1.}

After proving Corollaries 4.10 and 4.11, we learned that those results were already known in greater generality: they are consequences of [But94, Theorems 2.1 and 1]. Nevertheless, for elliptic curves over an algebraically closed field of characteristic zero, Theorem 4.9 is not a consequence of the results in [But94], and suggests that the following might be true: if $C$ is a smooth projective curve of genus $g$ and $\mathcal{U}$ and $\mathcal{V}$ are semistable locally free $\mathcal{O}_{C}$-modules such that $\mu(\mathcal{U})^{-1}+\mu(\mathcal{V})^{-1}<g^{-1}$, then the map $H^{0}(\mathcal{U}) \otimes H^{0}(\mathcal{V}) \rightarrow H^{0}(\mathcal{U} \otimes \mathcal{V})$ is surjective.

\subsection{2.}

In order for the multiplication map $H^{0}(\mathcal{U}) \otimes H^{0}(\mathcal{V}) \rightarrow H^{0}(\mathcal{U} \otimes \mathcal{V})$ in Theorem 4.9 to be onto, it is necessary that $\mu(\mathcal{U})^{-1}+\mu(\mathcal{V})^{-1}$ be $\leq 1$ : if the multiplication map is onto, then

$$
\operatorname{deg}(\mathcal{U} \otimes \mathcal{V})=\operatorname{dim} H^{0}(\mathcal{U} \otimes \mathcal{V}) \leq \operatorname{dim} H^{0}(\mathcal{U}) \operatorname{dim} H^{0}(\mathcal{V})=\operatorname{deg}(\mathcal{U}) \operatorname{deg}(\mathcal{V})
$$

since $\operatorname{deg}(\mathcal{U} \otimes \mathcal{V})=\operatorname{deg}(\mathcal{U}) \operatorname{rank}(\mathcal{V})+\operatorname{deg}(\mathcal{V}) \operatorname{rank}(\mathcal{U})$, $\operatorname{dividing}$ equation (4.3) by $\operatorname{deg}(\mathcal{U}) \operatorname{deg}(\mathcal{V})$ yields the inequality $\mu(\mathcal{U})^{-1}+\mu(\mathcal{V})^{-1} \leq 1$.

\subsection{3.}

There is a less elementary proof of the indecomposability of $\mathcal{K}$ in the proof of Theorem 4.9. Given a coherent sheaf $\mathcal{E}$ on $E$, let $T_{\mathcal{E}}$ be the endofunctor of the bounded derived category $\mathrm{D}^{b}(E)=\mathrm{D}^{b}(\operatorname{coh}(E))$ that sends $\mathcal{F}$ to the cone over

$$
\operatorname{RHom}(\mathcal{E}, \mathcal{F}) \otimes^{L} \mathcal{E} \rightarrow \mathcal{F}
$$

Applying this with $\mathcal{E}=\mathcal{O}$ yields $T_{\mathcal{O}}(\mathcal{U})=\mathcal{K}[1]$. But [ST01, Proposition 2.10] implies that $T_{\mathcal{O}}$ is an autoequivalence, so the indecomposability of $\mathcal{K}[1]$, and hence of $\mathcal{K}$, follows from that of $\mathcal{U}$.

\subsection{4.}

Lemma 4.8(4) with a stronger assumption of $\mu(\mathcal{F}) \geq 2$ can be shown using the following result, which might prove useful in other situations:

Lemma 4.12. Every semistable locally free $\mathcal{O}_{E}$-module $\mathcal{F}$ has a filtration by invertible $\mathcal{O}_{E}$-modules of degrees $\geq\lfloor\mu(\mathcal{F})\rfloor$. 
Proof. We can assume that $\mathcal{F}$ is indecomposable. Let $r=\operatorname{rank}(\mathcal{F})$ and $d=\operatorname{deg}(\mathcal{F})$. We will prove the result by induction on $r$. The result is certainly true when $r=1$.

If $\mu(\mathcal{F})=0$, then the assertion follows from [Ati57, Theorem 5].

If $0<\mu(\mathcal{F})<1$ (i.e., $0<d<r$ ), then [Ati57, Lemma 15] implies that $\mathcal{F}$ contains a rank- $d$ free subsheaf $\mathcal{I}$ of $\mathcal{F}$ such that $\mathcal{F} / \mathcal{I}$ is an indecomposable locally free sheaf of rank $r-d$ and degree $d$. The induction hypothesis shows that $\mathcal{F} / \mathcal{I}$ has a filtration by invertible sheaves of degree $\geq 0$. Thus $\mathcal{F}$ also has a filtration by invertible sheaves of degree $\geq 0=\lfloor\mu(\mathcal{F})\rfloor$.

If $\mu(\mathcal{F})<0$ or $1 \leq \mu(\mathcal{F})$, take an invertible sheaf $\mathcal{M}$ of degree $\lfloor\mu(\mathcal{F})\rfloor$. Then

$$
\mu\left(\mathcal{F} \otimes \mathcal{M}^{-1}\right)=\mu(\mathcal{F})-\mu(\mathcal{M})=\mu(\mathcal{F})-\lfloor\mu(\mathcal{F})\rfloor .
$$

The former two cases shows that $\mathcal{F} \otimes \mathcal{M}^{-1}$ admits a filtration by invertible sheaves of degrees $\geq 0$. Tensoring $\mathcal{M}$ with the filtration gives the desired filtration of $\mathcal{F}$. This completes the induction.

Let $\mathcal{F}$ be a semistable locally free $\mathcal{O}_{E}$-module of slope $\geq 2$. By Lemma $4.12, \mathcal{F}$ has a filtration

$$
0=\mathcal{Z}_{0} \subset \mathcal{Z}_{1} \subset \cdots \subset \mathcal{Z}_{r}=\mathcal{F}
$$

in which each $\mathcal{F}_{i}:=\mathcal{Z}_{i} / \mathcal{Z}_{i-1}$ is an invertible $\mathcal{O}_{E}$-module of degree $\geq 2$. All $H^{1}\left(\mathcal{F}_{i}\right)$ vanish, so an induction argument shows that all $H^{1}\left(\mathcal{Z}_{i}\right)$ vanish. Thus we obtain a commutative diagram

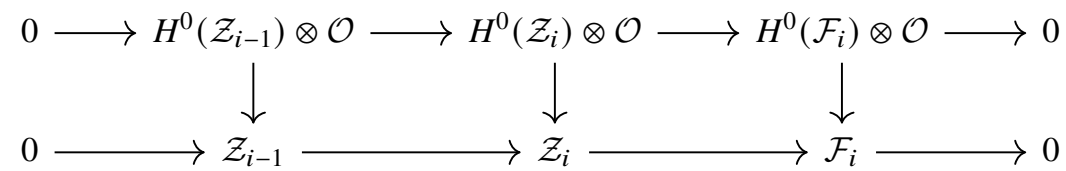

with exact rows. Since invertible sheaves of degree $\geq 2$ are generated by their global sections [Har77, Corollary IV.3.2], it is shown inductively that all $\mathcal{Z}_{i}$ are also generated by global sections using the diagram in (4.4). In particular, $\mathcal{F}$ is generated by global sections.

\section{Twisted homogeneous coordinate rings of the form $B\left(S^{g} E, \sigma, \mathcal{L}\right)$}

Let $\Sigma_{d}$ denote the symmetric group on $d$ letters. Let $\Sigma_{d}$ act on $E^{d}$ by having the transposition $(i, j)$ interchange the $i$ th and $j$ th coordinates $z_{i}$ and $z_{j}$ of a point $\left(z_{1}, \ldots, z_{d}\right) \in E^{d}$. The $d$ th symmetric power $S^{d} E$ is defined to be the quotient variety $E^{d} / \Sigma_{d}$ with respect to this action. We write

$$
\left(\left(z_{1}, \ldots, z_{d}\right)\right)
$$

for the image of $\left(z_{1}, \ldots, z_{d}\right)$ in $S^{d} E \cdot{ }^{10}$ As is well known, the addition map

$$
\pi=\operatorname{sum}: S^{d} E \rightarrow E, \quad \operatorname{sum}\left(\left(z_{1}, \ldots, z_{d}\right)\right)=z_{1}+\cdots+z_{d},
$$

(the Abel-Jacobi map) presents $S^{d} E$ as a $\mathbb{P}^{d-1}$-bundle over $E$. We will say more about this in $\S 5.3$.

For this reason we start this section with results about projective space bundles on $E$.

\subsection{Projective space bundles $\mathbb{P}(\mathcal{E})$ on an elliptic curve $E$}

We recall some standard results and notation for projective space bundles, for the most part following the material in [Har77, pp. 160-171].

\footnotetext{
${ }^{10}$ Sometimes $X_{n / k}$ is isomorphic to a symmetric power of $E$; under a careless identification between the two, the morphism $\Phi_{n / k}: E^{g} \rightarrow X_{n / k}$ might not correspond to the natural map $E^{g} \rightarrow S^{g} E$. This is irrelevant in this section and the next, but becomes relevant in section 7 .
} 
We adopt the following notation in this subsection:

$\circ \mathcal{E}$ is a locally free $\mathcal{O}_{E}$-module of rank $d$.

- $S^{m} \mathcal{E}$ is the $m$ th symmetric power of $\mathcal{E}$ when $m \geq 0$.

- $S(\mathcal{E})=\mathcal{O}_{E} \oplus \mathcal{E} \oplus S^{2} \mathcal{E} \oplus \cdots$ is the symmetric algebra on $\mathcal{E}$.

$\circ X=\mathbb{P}(\mathcal{E}):=\operatorname{Proj}(S(\mathcal{E}))$ is the associated $\mathbb{P}^{d-1}$-bundle on $E$.

○ $\mathcal{O}_{X}(1)$, or simply $\mathcal{O}(1)$, is the tautological $\mathcal{O}_{X}$-module associated to $S(\mathcal{E})$ - that is, $\pi_{*}\left(\mathcal{O}_{X}(1)\right)=\mathcal{E}$. $\circ \pi: \mathbb{P}(\mathcal{E}) \rightarrow E$ is the structure morphism.

We call $X$ the projectivisation of $\mathcal{E}$.

It follows from the definition of $\mathcal{O}_{X}(1)$ that there are canonical isomorphisms

$$
\pi_{*}\left(\mathcal{O}_{X}(m)\right) \cong S^{m} \mathcal{E}
$$

for all $m \geq 0$ (cf. [Har77, Proposition II.7.11]). We will make frequent use of this fact without further comment.

We will also make frequent use of the following observation:

Lemma 5.1. Assume char $(\mathbb{k})=0$. For all integers $m, n \geq 1$, the canonical maps $\mathcal{E}^{\otimes n} \rightarrow S^{n} \mathcal{E}$ and $S^{m} \mathcal{E} \otimes S^{n} \mathcal{E} \rightarrow S^{m+n} \mathcal{E}$ are split epimorphisms.

Proof. The map $\mathcal{E}^{\otimes n} \rightarrow S^{n} \mathcal{E}$ splits because char $(\mathbb{k})=0$. The composition

$$
\mathcal{E}^{\otimes(m+n)}=\mathcal{E}^{\otimes m} \otimes \mathcal{E}^{\otimes n} \longrightarrow S^{m} \mathcal{E} \otimes S^{n} \mathcal{E} \longrightarrow S^{m+n} \mathcal{E}
$$

is therefore a split epimorphism for all $m, n \geq 0$. The map $S^{m} \mathcal{E} \otimes S^{n} \mathcal{E} \rightarrow S^{m+n} \mathcal{E}$ is therefore a split epimorphism.

\subsubsection{Remark}

When $\operatorname{char}(\mathbb{k})=0$, the splitting of the $\operatorname{map} S^{m} \mathcal{E} \otimes S^{n} \mathcal{E} \rightarrow S^{m+n} \mathcal{E}$ can be defined universally by splitting the symmetrisation morphism $S^{m} \otimes S^{n} \rightarrow S^{m+n}$ in the category of polynomial endofunctors on the category $\operatorname{coh}(E)$ of coherent $\mathcal{O}_{E}$-modules (see, e.g., [SS15, §2.2] for a reminder on these).

Concretely, we write $T_{m, n}: S^{m+n} \rightarrow S^{m} \otimes S^{n}$ for the natural transformation between polynomial functors which for symmetric powers of a vector space $V$ reads

$$
S^{m+n} V \ni v_{1} \cdots v_{m+n} \mapsto \frac{m ! n !}{(m+n) !} \sum v_{a_{1}} \cdots v_{a_{m}} \otimes v_{b_{1}} \cdots v_{b_{n}} \in S^{m} V \otimes S^{n} V,
$$

where the sum is over all decompositions of the set $\{1, \cdots, m+n\}$ as a disjoint union of $\left\{a_{i}\right\}$ and $\left\{b_{j}\right\}$.

\subsection{2.}

Sometimes it is convenient to replace $\mathcal{E}$ by another invertible $\mathcal{O}_{E}$-module $\mathcal{E}^{\prime}$ such that $\mathbb{P}(\mathcal{E}) \cong \mathbb{P}\left(\mathcal{E}^{\prime}\right)$ as bundles over $E$. This matter is addressed in [Har77, Lemma II.7.9, p. 161, and Exercise II.7.9(b), p. 170]: if $\mathcal{E}$ and $\mathcal{E}^{\prime}$ are locally free $\mathcal{O}_{E}$-modules and $\pi: \mathbb{P}(\mathcal{E}) \rightarrow E$ and $\pi^{\prime}: \mathbb{P}\left(\mathcal{E}^{\prime}\right) \rightarrow E$ are the structure morphisms, then there is an isomorphism

$$
\varphi: X=\mathbb{P}(\mathcal{E}) \longrightarrow X^{\prime}=\mathbb{P}\left(\mathcal{E}^{\prime}\right)
$$

such that $\pi=\pi^{\prime} \varphi$ if and only if $\mathcal{E}^{\prime} \cong \mathcal{E} \otimes \mathcal{L}$ for some invertible $\mathcal{O}_{E}$-module $\mathcal{L}$. When this happens,

$$
\mathcal{O}_{X}(1) \cong \varphi^{*} \mathcal{O}_{X^{\prime}}(1) \otimes \pi^{*} \mathcal{L}
$$

Replacing $\mathcal{E}$ by $\mathcal{E}^{\prime}$ allows us to assume that $0 \leq \operatorname{deg}(\mathcal{E}) \leq \operatorname{rank}(\mathcal{E})-1-$ that is, given any $\mathcal{E}$, there is an invertible $\mathcal{O}_{E}$-module $\mathcal{L}$ such that $0 \leq \operatorname{deg}(\mathcal{E} \otimes \mathcal{L}) \leq \operatorname{rank}(\mathcal{E} \otimes \mathcal{L})-1$. 


\subsubsection{The Néron-Severi and Picard groups of $S^{d} E$}

There is a split exact sequence

$$
0 \longrightarrow \operatorname{Pic}(E) \stackrel{\pi^{*}}{\longrightarrow} \operatorname{Pic}(X) \longrightarrow \mathbb{Z} \longrightarrow 0
$$

with a splitting $\mathbb{Z} \rightarrow \operatorname{Pic}(X)$ given by $1 \mapsto\left[\mathcal{O}_{X}(1)\right]$ (see, e.g., [Har77, Exercise II.7.9(a) and A11, p. 429]).

The image in $\mathbb{Z}$ of $[\mathcal{L}] \in \operatorname{Pic}(X)$ is called the degree of $\mathcal{L}$ and is denoted by $\operatorname{deg}(\mathcal{L})$.

The Néron-Severi group $\operatorname{NS}(X)$ is isomorphic to $\mathbb{Z} \oplus \mathbb{Z}$ with basis $D:=\left[\mathcal{O}_{X}(1)\right]$ and $F=\left[F_{u}\right]$, where $F_{u}:=\pi^{-1}(u)$ is the fibre over an arbitrary point $u \in E$. It is well known that

$$
F \cdot F=0, \quad F \cdot D^{d-1}=1, \quad D^{d}=\operatorname{deg}(\mathcal{E})
$$

(see, e.g., [Gus90, Proposition 1.1(1)]. Let $D_{u}:=$ the image of $\{u\} \times E^{d-1}$ in $S^{d} E-$ that is, $D_{u}$ consists of the points $\left(\left(u, x_{2}, \ldots, x_{d}\right)\right)$ and is isomorphic to $S^{d-1} E$. When $X=S^{d} E, D=\left[\mathcal{O}_{X}(1)\right]=\left[D_{u}\right] .{ }^{11}$

Proposition 5.2. If $\mathcal{L}$ is an invertible $\mathcal{O}_{X}$-module, there is an invertible $\mathcal{O}_{E}$-module $\mathcal{L}^{\prime}$ such that

$$
\begin{aligned}
\mathcal{L} & \cong \mathcal{O}_{X}(a) \otimes \pi^{*} \mathcal{L}^{\prime}, \\
{[\mathcal{L}] } & =a D+\left(\operatorname{deg}\left(\mathcal{L}^{\prime}\right)\right) F,
\end{aligned}
$$

where $a=\operatorname{deg}(\mathcal{L})$.

Proposition 5.3 (Gushel [Gus90]). Let $\mathcal{E}$ be an indecomposable locally free $\mathcal{O}_{E}$-module of rank $d$ such that $0 \leq \operatorname{deg}(\mathcal{E}) \leq d-1 .{ }^{12}$ Let $\mathcal{L}$ be an invertible $\mathcal{O}_{X}$-module. Suppose $[\mathcal{L}]=a D+b F$. Then $\mathcal{L}$ is

(1) generated by its global sections if $a \geq 0$ and $b \geq 2$,

(2) ample if $a \geq 1$ and $b \geq 1$,

(3) very ample if $a \geq 1$ and $b \geq 3$.

Proof. These statements are weak versions of [Gus90, Proposition 1.1(iv), Proposition 3.3(i) and Theorem 4.3].

\subsection{4.}

In order to analyse the push-forward $\pi_{*} \mathcal{L}$ of an invertible $\mathcal{O}_{X}$-module $\mathcal{L}$, we adapt [Har77, Lemmas V.2.1 and V.2.4] to the present setting.

Lemma 5.4. Let $\mathcal{L}$ be an invertible $\mathcal{O}_{X}$-module with $[\mathcal{L}]=a D+b F \in \operatorname{NS}(X)$. If $a \geq 0$, then

(1) $\pi_{*} \mathcal{L}$ is a locally free $\mathcal{O}_{E}$-module of rank $\left(\begin{array}{c}a+d-1 \\ d-1\end{array}\right)$,

(2) $R^{i} \pi_{*}(\mathcal{L})=0$ for all $i \geq 1$,

(3) $H^{i}(X, \mathcal{L}) \cong H^{i}\left(E, \pi_{*} \mathcal{L}\right)$ for all $i \geq 0$.

Proof. For all $u \in E$, the restriction $\mathcal{L}_{u}$ of $\mathcal{L}$ to $F_{u}$ is isomorphic to $\mathcal{O}_{\mathbb{P} d-1}(a)$.

(1) The dimension of $H^{0}\left(F_{u}, \mathcal{L}_{u}\right)$ is therefore $\left(\begin{array}{c}a+d-1 \\ d-1\end{array}\right)$. Since this holds for all $u \in E$, the conclusion follows from Grauert's theorem [Har77, Corollary III.12.9], just as in the proof of [Har77, Lemma V.2.1].

(2) Since $a \geq 0, H^{i}\left(F_{u}, \mathcal{L}_{u}\right)=0$ for all $i \geq 1$ and $u \in E$. Thus Grauert's theorem shows that $R^{i} \pi_{*}(\mathcal{L}) \cong H^{i}\left(F_{u}, \mathcal{L}_{u}\right)=0$.

\footnotetext{
${ }^{11}$ The remarks after [CC93, Lemma 1.3] provide a nice proof of this equality that uses the Poincaré bundle on $E$. Here is another proof: clearly $\mathcal{O}_{X}\left(D_{u}\right)$ restricts to $\mathcal{O}_{F_{v}}(1)$ on every fibre $F_{v}$. The difference $\mathcal{R}:=\mathcal{O}_{X}\left(D_{u}\right)^{-1} \otimes \mathcal{O}_{X}(1)$ is therefore trivial on each fibre and hence a pullback of a divisor on $E$. It follows that in NS $(X), D=\left[D_{u}\right]+t F$ for some integer $t$. To show that $t=0$, it suffices to show that $D_{u}^{d}=1$. Intersections behave well in families, so we can compute $D_{u}^{d}$ by taking the intersection of $D_{u_{i}}$ for $d$ distinct points $u_{i}$; this intersection is obviously a singleton (and the intersections are transverse), so we conclude that $D_{u}^{d}=1$. Thus $t=0$ and $D=\left[D_{u}\right]$.

${ }^{12}$ As remarked in $\$ 5.1 .2$, if $\mathcal{E}$ is any indecomposable locally free $\mathcal{O}_{E}$-module of rank $d$, there is an indecomposable locally free $\mathcal{O}_{E}$-module $\mathcal{E}^{\prime}$ such that $X=\mathbb{P}(\mathcal{E})$ is isomorphic to $\mathbb{P}\left(\mathcal{E}^{\prime}\right)$.
} 
(3) By (2), the spectral sequence $H^{p}\left(E, R^{q} \pi_{*}(\mathcal{L})\right) \Rightarrow H^{p+q}(X, \mathcal{L})$ collapses. Thus, as in [Har77, Lemma V.2.4], $H^{i}\left(E, \pi_{*} \mathcal{L}\right) \cong H^{i}(X, \mathcal{L})$ for all $i \geq 0$.

\subsection{Multiplication of sections of invertible sheaves on $\mathbb{P}(\mathcal{E})$}

We continue to assume that $X=\mathbb{P}(\mathcal{E})$ and keep the notations in $\$ 5.1$.

Lemma 5.5. Assume char $(\mathbb{k})=0$. Let $\mathcal{L}_{1}=\mathcal{O}_{X}\left(a_{1}\right) \otimes \pi^{*} \mathcal{L}_{1}^{\prime}$ and $\mathcal{L}_{2}=\mathcal{O}_{X}\left(a_{2}\right) \otimes \pi^{*} \mathcal{L}_{2}^{\prime}$ be invertible $\mathcal{O}_{X}$-modules. If $a_{1}$ and $a_{2}$ are $\geq 0$, then the natural map

$$
\pi_{*} \mathcal{L}_{1} \otimes \pi_{*} \mathcal{L}_{2} \longrightarrow \pi_{*}\left(\mathcal{L}_{1} \otimes \mathcal{L}_{2}\right)
$$

is a split epimorphism.

Proof. By the Projection Formula [Har77, p. 124],

$$
\pi_{*}\left(\mathcal{L}_{i}\right)=\pi_{*}\left(\mathcal{O}\left(a_{i}\right) \otimes \pi^{*} \mathcal{L}_{i}^{\prime}\right) \cong \pi_{*}\left(\mathcal{O}\left(a_{i}\right)\right) \otimes \mathcal{L}_{i}^{\prime} \cong S^{a_{i}} \mathcal{E} \otimes \mathcal{L}_{i}^{\prime}
$$

Similarly, $\pi_{*}\left(\mathcal{L}_{1} \otimes \mathcal{L}_{2}\right) \cong S^{a_{1}+a_{2}} \mathcal{E} \otimes \mathcal{L}_{1}^{\prime} \otimes \mathcal{L}_{2}^{\prime}$. The morphism in (5.1) can therefore be written as

$$
S^{a_{1}} \mathcal{E} \otimes \mathcal{L}_{1}^{\prime} \otimes S^{a_{2}} \mathcal{E} \otimes \mathcal{L}_{2}^{\prime} \longrightarrow S^{a_{1}+a_{2}} \mathcal{E} \otimes \mathcal{L}_{1}^{\prime} \otimes \mathcal{L}_{2}^{\prime}
$$

It follows from Lemma 5.1 that this is a split epimorphism.

Proposition 5.6. Assume $\operatorname{char}(\mathbb{k})=0$. Let $\mathcal{E}$ be a semistable locally free $\mathcal{O}_{E}$-module of positive degree and let $X=\mathbb{P}(\mathcal{E})$. Let $\mathcal{L}$ and $\mathcal{F}$ be invertible $\mathcal{O}_{X}$-modules whose classes in $\mathrm{NS}(X)$ are $a D+b F$ and $s D+t F$, respectively. If $a, s \geq 1$ and $b, t \geq 2$, then the multiplication maps

(1) $H^{0}\left(E, \pi_{*} \mathcal{L}\right) \otimes H^{0}\left(E, \pi_{*} \mathcal{F}\right) \longrightarrow H^{0}\left(E, \pi_{*} \mathcal{L} \otimes \pi_{*} \mathcal{F}\right)$ and

(2) $H^{0}(X, \mathcal{L}) \otimes H^{0}(X, \mathcal{F}) \longrightarrow H^{0}(X, \mathcal{L} \otimes \mathcal{F})$

are onto.

Proof. (1) Let $\mathcal{U}=\pi_{*} \mathcal{L}$ and $\mathcal{V}=\pi_{*} \mathcal{F}$.

By Proposition 5.2, there are invertible $\mathcal{O}_{E}$-modules $\mathcal{L}^{\prime}$ and $\mathcal{F}^{\prime}$, of degrees $b$ and $t$, respectively, such that $\mathcal{L} \cong \mathcal{O}_{X}(a) \otimes \pi^{*}\left(\mathcal{L}^{\prime}\right)$ and $\mathcal{F} \cong \mathcal{O}_{X}(s) \otimes \pi^{*}\left(\mathcal{F}^{\prime}\right)$. By the Projection Formula, $\mathcal{U} \cong \pi_{*}\left(\mathcal{O}_{X}(a)\right) \otimes \mathcal{L}^{\prime}$ and $\mathcal{V} \cong \pi_{*}\left(\mathcal{O}_{X}(s)\right) \otimes \mathcal{F}^{\prime}$

Since $\mathcal{E}$ is semistable, Lemma 4.6 tells us that $\mathcal{E}^{\otimes a}$ is semistable too; its direct summand $S^{a} \mathcal{E}$ is therefore semistable too. Similarly, $S^{s} \mathcal{E}$ is semistable. Since $\mathcal{L}^{\prime}$ and $\mathcal{F}^{\prime}$ are semistable by Lemma 4.5, the tensor products $\mathcal{U}$ and $\mathcal{V}$ are also semistable by Lemma 4.6.

Since $\mathcal{E}$ has positive degree, by assumption, and $a, s \geq 1$, the summands $S^{a} \mathcal{E}$ and $S^{s} \mathcal{E}$ of the semistable sheaves $\mathcal{E}^{\otimes a}$ and $\mathcal{E}^{\otimes s}$ have positive degree too. Hence

$$
\mu(\mathcal{U})=\mu\left(\pi_{*}\left(\mathcal{O}_{X}(a)\right)\right)+\mu\left(\mathcal{L}^{\prime}\right)=\mu\left(S^{a} \mathcal{E}\right)+\mu\left(\mathcal{L}^{\prime}\right)>\mu\left(\mathcal{L}^{\prime}\right) \geq 2 .
$$

Similarly, $\mu(\mathcal{V})>2$. The result now follows from Corollary 4.11.

(2) The canonical map $H^{0}\left(\pi_{*} \mathcal{L}\right) \otimes H^{0}\left(\pi_{*} \mathcal{F}\right) \rightarrow H^{0}\left(\pi_{*}(\mathcal{L} \otimes \mathcal{F})\right)$ factors as

$$
H^{0}\left(E, \pi_{*} \mathcal{L}\right) \otimes H^{0}\left(E, \pi_{*} \mathcal{F}\right) \longrightarrow H^{0}\left(E, \pi_{*} \mathcal{L} \otimes \pi_{*} \mathcal{F}\right) \longrightarrow H^{0}\left(E, \pi_{*}(\mathcal{L} \otimes \mathcal{F})\right) .
$$

We have just shown that the leftmost map in this composition is onto; the other is also onto, because the canonical map $\pi_{*} \mathcal{L} \otimes \pi_{*} \mathcal{F} \rightarrow \pi_{*}(\mathcal{L} \otimes \mathcal{F})$ is a split epimorphism by Lemma 5.5 . The composition is therefore onto. The result now follows from the fact that there are functorial isomorphisms $H^{0}(X, \mathcal{L}) \cong$ $H^{0}\left(E, \pi_{*} \mathcal{L}\right), H^{0}(X, \mathcal{F}) \cong H^{0}\left(E, \pi_{*} \mathcal{F}\right)$ and $H^{0}(X, \mathcal{L} \otimes \mathcal{F}) \cong H^{0}\left(E, \pi_{*}(\mathcal{L} \otimes \mathcal{F})\right)$. 


\subsection{Symmetric powers of $E$}

Let $X=S^{d} E$.

Up to tensoring with a degree- 0 invertible sheaf, there is a unique indecomposable locally free sheaf $\mathcal{E}$ of rank $d$ and degree 1 on $E$ such that $S^{d} E \cong \mathbb{P}(\mathcal{E})$ [Ati57, p. 451]. By [Ati57, Theorem 5, p. 432], one can construct such an $\mathcal{E}$ iteratively: let $\mathcal{E}_{1}$ be any invertible $\mathcal{O}_{E}$-module of degree one and for $r \geq 2$, let $\mathcal{E}_{r}$ be the 'unique' nontrivial extension $0 \rightarrow \mathcal{O}_{E} \rightarrow \mathcal{E}_{r} \rightarrow \mathcal{E}_{r-1} \rightarrow 0$. Thus $X=S^{d} E=\mathbb{P}\left(\mathcal{E}_{d}\right)$ and $\pi_{*}\left(\mathcal{O}_{X}(1)\right) \cong \mathcal{E}_{d}$. Since $\mathcal{E}_{d}$ is indecomposable, it is semistable (in fact, stable, because its degree is 1 ) of positive degree, so Proposition 5.6 applies.

Whenever we view $S^{d} E$ as a projective bundle $\mathbb{P}(\mathcal{E})$, we will assume $\mathcal{E}$ is $\mathcal{E}_{d}$.

See [Ati57, p. 451], [CC93], and [Po105] for more information about $S^{d} E$ as a projective space bundle.

Theorem 5.7. Assume $\operatorname{char}(\mathbb{k})=0$. Let $\sigma: S^{d} E \rightarrow S^{d} E$ be a translation automorphism. Let $\mathcal{L}$ be an invertible sheaf on $S^{d} E$ whose Néron-Severi class is $[\mathcal{L}]=a D+b F$. If $a \geq 1$ and $b \geq 2$, then $B\left(S^{d} E, \sigma, \mathcal{L}\right)$ is generated in degree one.

Proof. By definition, the degree- $n$ homogeneous component of $B\left(S^{d} E, \sigma, \mathcal{L}\right)$ is

$$
B_{n}=H^{0}\left(S^{d} E, \mathcal{L} \otimes \cdots \otimes\left(\sigma^{*}\right)^{n-1} \mathcal{L}\right) .
$$

The surjectivity of the multiplication map $B_{1} \otimes B_{n} \rightarrow B_{n+1}$ therefore follows from Proposition 5.6 applied to $\mathcal{L}$ and $\mathcal{F}=\sigma^{*} \mathcal{L} \otimes \cdots \otimes\left(\sigma^{*}\right)^{n} \mathcal{L}$.

\section{Relations for $B\left(S^{g} E, \sigma, \mathcal{L}\right)$}

The main result in this section, Theorem 6.9, shows that rather mild hypotheses imply that the relations for $B\left(S^{g} E, \sigma, \mathcal{L}\right)$ are generated in degrees 2 and 3.

\subsection{Preparations}

The following hypotheses and notation apply throughout this section:

$\circ \mathbb{k}$ is an algebraically closed field of characteristic zero.

$\circ \pi: S^{g} E \rightarrow E$ is the map $\pi\left(\left(z_{1}, \ldots, z_{g}\right)\right)=z_{1}+\cdots+z_{g}$.

$\circ \sigma$ is an arbitrary translation automorphism of $S^{g} E$.

$\circ \mathcal{L}$ is an invertible $\mathcal{O}_{S^{g}} E$-module such that $[\mathcal{L}]=a D+b F$, where $a \geq 1$ and $b \geq 2$.

○ $X=S^{g} E, g \geq 2$.

By Proposition 5.3, $\mathcal{L}$ is ample and is generated by its global sections.

By Theorem 5.7, $B:=B\left(S^{g} E, \sigma, \mathcal{L}\right)$ is generated as a $\mathbb{k}$-algebra by its degree-one component $B_{1}$ - that is, the canonical $\mathbb{k}$-algebra homomorphism $\varphi: T\left(B_{1}\right) \rightarrow B$ from the tensor algebra on $B_{1}$ is surjective.

\subsection{1.}

To prove that the ideal $J:=\operatorname{ker}(\varphi)$ is generated by $J_{2}+J_{3}$, it suffices, by Lemma 2.11, to show that the canonical map

$$
H^{0}\left(S^{g} E, \mathcal{L}\right) \otimes R\left(\mathcal{M}_{m}, \mathcal{N}_{m}\right) \longrightarrow R\left(\mathcal{L} \otimes \mathcal{M}_{m}, \mathcal{N}_{m}\right)
$$

is surjective for all $m \geq 2$, where $\mathcal{M}_{m}$ and $\mathcal{N}_{m}$ are the sheaves defined at the beginning of $\S 2.5$. We will prove more: if $\mathcal{L}, \mathcal{M}=\mathcal{M}_{m}$ and $\mathcal{N}=\mathcal{N}_{m}$ have the properties in Convention 6.1, then the canonical map

$$
H^{0}\left(S^{g} E, \mathcal{L}\right) \otimes R(\mathcal{M}, \mathcal{N}) \longrightarrow R(\mathcal{L} \otimes \mathcal{M}, \mathcal{N})
$$

is onto. 
Convention 6.1. Let $E$ be an elliptic curve and let $X=S^{g}$ E. Let $\mathcal{L}, \mathcal{M}$ and $\mathcal{N}$ be invertible $\mathcal{O}_{X}$-modules with Néron-Severi classes

$$
\begin{aligned}
& {[\mathcal{L}]=a D+b F \quad \text { where } a \geq 1 \text { and } b \geq 2 \text {, }} \\
& {[\mathcal{M}]=p D+q F \quad \text { where } p \geq 2 \text { and } q \geq 4 \text {, }} \\
& {[\mathcal{N}]=s D+t F \quad \text { where } s \geq 1 \text { and } t \geq 2 \text {. }}
\end{aligned}
$$

\subsection{Surjectivity of multiplication maps}

By Proposition 5.3, $\mathcal{N}$ is generated by its global sections. Let

$$
0 \rightarrow \mathcal{K} \rightarrow H^{0}\left(S^{g} E, \mathcal{N}\right) \otimes \mathcal{O}_{S^{g} E} \rightarrow \mathcal{N} \rightarrow 0
$$

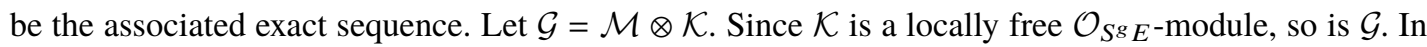
this case, the argument in Lemma 2.12 shows that map in (6.1) is onto if and only if the map

$$
H^{0}\left(S^{g} E, \mathcal{L}\right) \otimes H^{0}\left(S^{g} E, \mathcal{G}\right) \rightarrow H^{0}\left(S^{g} E, \mathcal{L} \otimes \mathcal{G}\right) .
$$

is onto.

The $\mathcal{O}_{S^{g}} E$-module $\mathcal{L}$ satisfies the hypotheses and therefore the conclusions of Lemma 5.4. Although $\mathcal{G}$ and $\mathcal{L} \otimes \mathcal{G}$ need not be invertible, they have similar properties.

\section{Lemma 6.2.}

(1) $\pi_{*} \mathcal{G}$ and $\pi_{*}(\mathcal{L} \otimes \mathcal{G})$ are locally free $\mathcal{O}_{E}$-modules.

(2) $R^{i} \pi_{*}(\mathcal{G})=R^{i} \pi_{*}(\mathcal{L} \otimes \mathcal{G})=0$ for all $i \geq 1$.

(3) $H^{i}\left(S^{g} E, \mathcal{G}\right) \cong H^{i}\left(E, \pi_{*} \mathcal{G}\right)$ and $H^{i}\left(S^{g} E, \mathcal{L} \otimes \mathcal{G}\right) \cong H^{i}\left(E, \pi_{*}(\mathcal{L} \otimes \mathcal{G})\right)$ for all $i \geq 0$.

Proof. We prove the lemma for $\mathcal{G}$. The other case is similar. Since $\mathcal{M}$ and $\mathcal{M} \otimes \mathcal{N}$ satisfy the hypotheses of Lemma 5.4, $R^{i} \pi_{*} \mathcal{M}=R^{i} \pi_{*}(\mathcal{M} \otimes \mathcal{N})=0$ for all $i \geq 1$. Hence the short exact sequence (6.2) tensored with $\mathcal{M}$ produces the exact sequence

$$
0 \longrightarrow \pi_{*} \mathcal{G} \longrightarrow \pi_{*}\left(\mathcal{M} \otimes H^{0}\left(S^{g} E, \mathcal{N}\right)\right) \stackrel{f}{\longrightarrow} \pi_{*}(\mathcal{M} \otimes \mathcal{N}) \longrightarrow R^{1} \pi_{*}(\mathcal{G}) \longrightarrow 0
$$

and shows $R^{i} \pi_{*} \mathcal{G}=0$ for all $i \geq 2$. Applying $H^{0}\left(S^{g} E,-\right)$, the morphism $f$ induces a map

$$
H^{0}\left(S^{g} E, \mathcal{M}\right) \otimes H^{0}\left(S^{g} E, \mathcal{N}\right) \rightarrow H^{0}\left(S^{g} E, \mathcal{M} \otimes \mathcal{N}\right),
$$

which is surjective by Proposition 5.6. Since $\pi_{*}(\mathcal{M} \otimes \mathcal{N})$ is semistable and has slope $\geq 2$, as shown in the proof of Proposition 5.6, it is generated by global sections by Lemma 4.8(4). Therefore the morphism $f$ is surjective. This implies $R^{1} \pi_{*}(\mathcal{G})=0$.

Since $\pi_{*}\left(\mathcal{M} \otimes H^{0}(\mathcal{N})\right)$ is a locally free $\mathcal{O}_{E}$-module, so is $\pi_{*} \mathcal{G}$. Assertion (3) follows in the same way as Lemma 5.4.

Lemma 6.3. The map in (6.3) is surjective if and only if the map

$$
H^{0}\left(E, \pi_{*} \mathcal{L}\right) \otimes H^{0}\left(E, \pi_{*} \mathcal{G}\right) \rightarrow H^{0}\left(E, \pi_{*}(\mathcal{L} \otimes \mathcal{G})\right)
$$

is surjective.

Proof. This is an immediate consequence of Lemma 6.2.

In what follows, we use Lemma 6.2 without further comment.

By Lemma 5.5, the map $\pi_{*} \mathcal{L}_{1} \otimes \pi_{*} \mathcal{L}_{2} \rightarrow \pi_{*}\left(\mathcal{L}_{1} \otimes \mathcal{L}_{2}\right)$ is a split epimorphism for suitable invertible $\mathcal{O}_{S^{g}} E^{- \text {modules }} \mathcal{L}_{i}$. The next result is analogous. 
Lemma 6.4. Under Convention 6.1, the canonical map

$$
\pi_{*} \mathcal{L} \otimes \pi_{*} \mathcal{G} \rightarrow \pi_{*}(\mathcal{L} \otimes \mathcal{G})
$$

is a split epimorphism and therefore induces a surjection on global sections over E.

Proof. There are invertible $\mathcal{O}_{E}$-modules $\mathcal{L}^{\prime}, \mathcal{M}^{\prime}$ and $\mathcal{N}^{\prime}$ of degrees $b \geq 2, q \geq 4$ and $t \geq 2$, respectively, such that

$$
\mathcal{L} \cong \mathcal{O}(a) \otimes \pi^{*}\left(\mathcal{L}^{\prime}\right), \quad \mathcal{M} \cong \mathcal{O}(p) \otimes \pi^{*}\left(\mathcal{M}^{\prime}\right), \quad \text { and } \quad \mathcal{N} \cong \mathcal{O}(s) \otimes \pi^{*}\left(\mathcal{N}^{\prime}\right)
$$

Since $0 \rightarrow \mathcal{G} \rightarrow \mathcal{M} \otimes H^{0}\left(S^{g} E, \mathcal{N}\right) \rightarrow \mathcal{M} \otimes \mathcal{N} \rightarrow 0$ is exact by the definition of $\mathcal{G}, \pi_{*} \mathcal{G}$ is the kernel of

$$
\pi_{*} \mathcal{O}(p) \otimes \mathcal{M}^{\prime} \otimes H^{0}\left(E, \pi_{*} \mathcal{O}(s) \otimes \mathcal{N}^{\prime}\right) \longrightarrow \pi_{*} \mathcal{O}(p+s) \otimes \mathcal{M}^{\prime} \otimes \mathcal{N}^{\prime}
$$

Similarly, $\pi_{*}(\mathcal{L} \otimes \mathcal{G})$ is the kernel of

$$
\pi_{*} \mathcal{O}(a+p) \otimes H^{0}\left(E, \pi_{*} \mathcal{O}(s) \otimes \mathcal{N}^{\prime}\right) \longrightarrow \pi_{*} \mathcal{O}(a+p+s) \otimes \mathcal{N}^{\prime}
$$

tensored with $\mathcal{L}^{\prime} \otimes \mathcal{M}^{\prime}$. Tensoring the map in (6.6) with $\pi_{*} \mathcal{L}$ produces the tensor product of

$$
\pi_{*} \mathcal{O}(a) \otimes \pi_{*} \mathcal{O}(p) \otimes H^{0}\left(E, \pi_{*} \mathcal{O}(s) \otimes \mathcal{N}^{\prime}\right) \longrightarrow \pi_{*} \mathcal{O}(a) \otimes \pi_{*} \mathcal{O}(p+s) \otimes \mathcal{N}^{\prime}
$$

with $\mathcal{L}^{\prime} \otimes \mathcal{M}^{\prime}$; in other words, tensoring (6.6) with $\pi_{*} \mathcal{L}$ produces

$$
\pi_{*} \mathcal{L} \otimes \pi_{*} \mathcal{M} \otimes H^{0}\left(E, \pi_{*} \mathcal{N}\right) \longrightarrow \pi_{*} \mathcal{L} \otimes \pi_{*}(\mathcal{M} \otimes \mathcal{N})
$$

The symmetrisation map $\pi_{*} \mathcal{O}(a) \otimes \pi_{*} \mathcal{O}(p) \rightarrow \pi_{*} \mathcal{O}(a+p)$ between the left-hand terms of (6.7) and (6.8) splits compatibly with the symmetrisation map between the right-hand terms. Indeed, this amounts to noting that the diagram

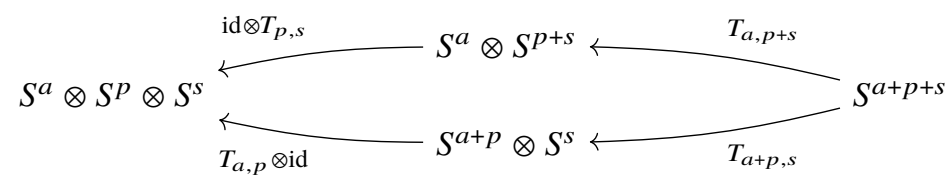

of functors defined as in $\$ 5.1 .1$ commutes. When applied to a finite-dimensional vector space $V$, the diagram of functors expresses the coassociativity of the shuffle comultiplication on the symmetric algebra $S V$ (with this bialgebra structure, $S V$ is the graded dual to the universal cocommutative bialgebra $B(V)$ defined in [Swe69, $\$ \$ 12.2$ and 12.3]).

In conclusion, the map in (6.7) can be realised as a direct summand of the map in (6.8). The kernel of the map in (6.7) tensored with $\mathcal{L}^{\prime} \otimes \mathcal{M}^{\prime}$, namely $\pi_{*}(\mathcal{L} \otimes \mathcal{G})$, is therefore a direct summand of the kernel of the map in (6.8) tensored with $\mathcal{L}^{\prime} \otimes \mathcal{M}^{\prime}$, and hence a direct summand of the kernel of the map in (6.9), which is $\pi_{*} \mathcal{L} \otimes \pi_{*} \mathcal{G}$.

By Lemma 6.3, the map in (6.3) is surjective if and only if the map in (6.5) is. Now by Lemma 6.4, the surjectivity of the map in (6.5) is equivalent to the surjectivity of

$$
H^{0}\left(E, \pi_{*} \mathcal{L}\right) \otimes H^{0}\left(E, \pi_{*} \mathcal{G}\right) \longrightarrow H^{0}\left(E, \pi_{*} \mathcal{L} \otimes \pi_{*} \mathcal{G}\right)
$$

This has the same flavor as Theorem 4.9, and we will use that result to obtain the conclusion.

Lemma 6.5. The locally free $\mathcal{O}_{E}$-module $\pi_{*} \mathcal{L}$ is isomorphic to $\pi_{*} \mathcal{O}(a) \otimes \mathcal{L}^{\prime}$, is semistable, is generated by its global sections and has slope $a \mu(\mathcal{E})+b$. 
Proof. We have argued along the same lines already, several times: $\pi_{*} \mathcal{O}_{S^{g}} E(1) \cong \mathcal{E}$ is a semistable $\mathcal{O}_{E^{-}}$ module, so the summand $\pi_{*} \mathcal{O}(a)$ of its tensor power $\pi_{*} \mathcal{O}(1)^{\otimes a}$ is semistable of slope $a \mu(\mathcal{E})$. Lemma 4.6 now tells us that $\pi_{*} \mathcal{O}(a) \otimes \mathcal{L}^{\prime}$ is also semistable.

The claim of global generation now follows from Proposition 5.3.

The slope of $\pi_{*} \mathcal{O}(a)$ is $a \mu(\mathcal{E})$, so

$$
\mu\left(\pi_{*} \mathcal{L}\right)=\mu\left(\pi_{*} \mathcal{O}(a) \otimes \mathcal{L}^{\prime}\right)=\mu\left(\pi_{*} \mathcal{O}(a)\right)+\mu\left(\mathcal{L}^{\prime}\right)=a \mu(\mathcal{E})+b,
$$

as claimed.

We now consider $\pi_{*} \mathcal{G}$. As noted in the proof of Lemma $6.4, \pi_{*} \mathcal{G}$ is the kernel of the composition

$$
H^{0}\left(\pi_{*} \mathcal{O}(s) \otimes \mathcal{N}^{\prime}\right) \otimes \pi_{*} \mathcal{O}(p) \otimes \mathcal{M}^{\prime} \rightarrow \pi_{*} \mathcal{O}(p) \otimes \pi_{*} \mathcal{O}(s) \otimes \mathcal{M}^{\prime} \otimes \mathcal{N}^{\prime} \rightarrow \pi_{*} \mathcal{O}(p+s) \otimes \mathcal{M}^{\prime} \otimes \mathcal{N}^{\prime}
$$

where the first morphism is the canonical map

$$
H^{0}\left(E, \pi_{*} \mathcal{O}(s) \otimes \mathcal{N}^{\prime}\right) \otimes \mathcal{O}_{E} \rightarrow \pi_{*} \mathcal{O}(s) \otimes \mathcal{N}^{\prime}
$$

tensored with $\pi_{*} \mathcal{O}(p) \otimes \mathcal{M}^{\prime}$. The argument of Lemma 6.5 shows that $\pi_{*} \mathcal{N} \cong \pi_{*} \mathcal{O}(s) \otimes \mathcal{N}^{\prime}$ is also generated by global sections, and hence the map in (6.11) is an epimorphism and so is the first morphism of the composition in (6.10). By Lemma 5.5, the second morphism is also an epimorphism. Thus there is an exact sequence

$$
0 \rightarrow \operatorname{ker}(\alpha) \rightarrow \pi_{*} \mathcal{G} \rightarrow \operatorname{ker}(\beta) \rightarrow 0,
$$

where $\alpha$ and $\beta$ are the epimorphisms

$$
\alpha: H^{0}\left(E, \pi_{*} \mathcal{O}(s) \otimes \mathcal{N}^{\prime}\right) \otimes \pi_{*} \mathcal{O}(p) \otimes \mathcal{M}^{\prime} \longrightarrow \pi_{*} \mathcal{O}(p) \otimes \pi_{*} \mathcal{O}(s) \otimes \mathcal{M}^{\prime} \otimes \mathcal{N}^{\prime}
$$

and

$$
\beta: \pi_{*} \mathcal{O}(p) \otimes \pi_{*} \mathcal{O}(s) \otimes \mathcal{M}^{\prime} \otimes \mathcal{N}^{\prime} \longrightarrow \pi_{*} \mathcal{O}(p+s) \otimes \mathcal{M}^{\prime} \otimes \mathcal{N}^{\prime}
$$

The morphism (6.13) is a split epimorphism, so $\operatorname{ker}(\beta)$ is semistable of slope

$$
\mu(\operatorname{ker}(\beta))=\mu\left(\pi_{*} \mathcal{O}(p) \otimes \pi_{*} \mathcal{O}(s) \otimes \mathcal{M}^{\prime} \otimes \mathcal{N}^{\prime}\right)=(p+s) \mu(\mathcal{E})+q+t
$$

On the other hand, $\operatorname{ker}(\alpha)$ is isomorphic to $T_{\mathcal{O}}\left(\pi_{*} \mathcal{O}(s) \otimes \mathcal{N}^{\prime}\right)[-1] \otimes \pi_{*} \mathcal{O}(p) \otimes \mathcal{M}^{\prime}$, with $T_{\mathcal{O}}$ as in $\S 4.3 .3$. We saw in passing in the proof of Theorem 4.9 that $T_{\mathcal{O}}\left(\pi_{*} \mathcal{O}(s) \otimes \mathcal{N}^{\prime}\right)[-1]$ is semistable and its slope $\mu$ satisfies

$$
1=-\frac{1}{\mu}+\frac{1}{\mu\left(\pi_{*} \mathcal{O}(s) \otimes \mathcal{N}^{\prime}\right)}=-\frac{1}{\mu}+\frac{1}{s \mu(\mathcal{E})+t} .
$$

Hence

$$
\mu=\frac{s \mu(\mathcal{E})+t}{1-s \mu(\mathcal{E})-t}
$$

In conclusion, we obtain

$$
\mu(\operatorname{ker}(\alpha))=\mu+\mu\left(\pi_{*} \mathcal{O}(p) \otimes \mathcal{M}^{\prime}\right)=\mu+p \mu(\mathcal{E})+q=\frac{s \mu(\mathcal{E})+t}{1-s \mu(\mathcal{E})-t}+p \mu(\mathcal{E})+q .
$$

Clearly, this is smaller than (6.14).

Lemma 6.6. The locally free $\mathcal{O}_{E}$-module $\pi_{*} \mathcal{G}$ is a direct sum of semistable summands of slopes $\geq(6.15)$ and is generated by global sections. 
Proof. Since $\pi_{*} \mathcal{G}$ is an extension of the semistable locally free sheaf $\operatorname{ker}(\beta)$ by the semistable locally free sheaf $\operatorname{ker}(\alpha)$, its semistable summands have slopes

$$
\geq \mu(\operatorname{ker}(\alpha))=\min (\mu(\operatorname{ker}(\alpha)), \mu(\operatorname{ker}(\beta))) .
$$

Since $\mu(\mathcal{E})=\mu\left(\mathcal{E}_{d}\right)=\frac{1}{d}$, we have $s \mu(\mathcal{E})+t>3$ and $p \mu(\mathcal{E})+q>4$. Thus

$$
\mu(\operatorname{ker}(\beta)) \geq \mu(\operatorname{ker}(\alpha))=\frac{1}{\frac{1}{s \mu(\mathcal{E})+t}-1}+p \mu(\mathcal{E})+q \geq-2+p \mu(\mathcal{E})+q>2 .
$$

By Lemma 4.8(4), $\operatorname{ker}(\alpha)$ and $\operatorname{ker}(\beta)$ are generated by global sections. Since $H^{1}(\operatorname{ker}(\alpha))=0$ by Lemma 4.8, the sheaves $\operatorname{ker}(\alpha), \pi_{*} \mathcal{G}$ and $\operatorname{ker}(\beta)$ form an analogous diagram to the diagram in (4.4), which shows the global generation of $\pi_{*} \mathcal{G}$.

Remark 6.7. This proof shows that $\mu(\operatorname{ker}(\alpha))>2$.

Lemma 6.8. Under the assumptions of Convention 6.1, the canonical map

$$
H^{0}\left(E, \pi_{*} \mathcal{L}\right) \otimes H^{0}\left(E, \pi_{*} \mathcal{G}\right) \longrightarrow H^{0}\left(E, \pi_{*} \mathcal{L} \otimes \pi_{*} \mathcal{G}\right)
$$

is onto.

Proof. This will follow from Lemmas 6.5 and 6.6 and Theorem 4.9 applied to the semistable summands of $\pi_{*} \mathcal{L}$ and $\pi_{*} \mathcal{G}$, once we show that $\mu(\operatorname{ker}(\alpha))$ satisfies

$$
\frac{1}{\mu(\operatorname{ker}(\alpha))}+\frac{1}{\mu\left(\pi_{*} \mathcal{L}\right)}=\frac{1}{\mu(\operatorname{ker}(\alpha))}+\frac{1}{a \mu(\mathcal{E})+b}<1 .
$$

This, however, is immediate from Remark 6.7, which shows that both summands on the left-hand side of equation (6.16) are less than $\frac{1}{2}$.

Theorem 6.9. Let $\sigma: S^{g} E \rightarrow S^{g} E$ be a translation automorphism. Let $\mathcal{L}$ be an invertible $\mathcal{O}_{S^{g} E^{-}}$ module that is ample and generated by its global sections. If $[\mathcal{L}]=a D+b F$ with $a \geq 1$ and $b \geq 2$, then $B\left(S^{g} E, \sigma, \mathcal{L}\right)$ is generated in degree one and has relations of degrees 2 and 3.

Proof. By Theorem 5.7, $B\left(S^{g} E, \sigma, \mathcal{L}\right)$ is generated in degree one.

Let $\mathcal{M}=\mathcal{M}_{m}$ and $\mathcal{N}=\mathcal{N}_{m}$ be as defined at the beginning of $\S 2.5$. Then $\mathcal{L}, \mathcal{M}_{m}$ and $\mathcal{N}_{m}$ satisfy the assumptions in Convention 6.1 for all $m \geq 2$. Let $\mathcal{G}=\mathcal{M} \otimes \mathcal{K}$, where $\mathcal{K}$ is the kernel in the exact sequence

$$
0 \rightarrow \mathcal{K} \rightarrow H^{0}\left(S^{g} E, \mathcal{N}\right) \otimes \mathcal{O}_{S^{g} E} \rightarrow \mathcal{N} \rightarrow 0
$$

Since $B\left(S^{g} E, \sigma, \mathcal{L}\right)$ is generated in degree one, to prove the theorem it suffices, by Lemma 2.12, to show that the multiplication map $H^{0}\left(S^{g} E, \mathcal{L}\right) \otimes H^{0}\left(S^{g} E, \mathcal{G}\right) \rightarrow H^{0}\left(S^{g} E, \mathcal{L} \otimes \mathcal{G}\right)$ is onto for all $m \geq 2$. By Lemma 6.3, this is onto if and only if the map $H^{0}\left(E, \pi_{*} \mathcal{L}\right) \otimes H^{0}\left(E, \pi_{*} \mathcal{G}\right) \rightarrow H^{0}\left(E, \pi_{*}(\mathcal{L} \otimes \mathcal{G})\right)$ is onto. This map factors as

$$
H^{0}\left(E, \pi_{*} \mathcal{L}\right) \otimes H^{0}\left(E, \pi_{*} \mathcal{G}\right) \longrightarrow H^{0}\left(E, \pi_{*} \mathcal{L} \otimes \pi_{*} \mathcal{G}\right) \longrightarrow H^{0}\left(E, \pi_{*}(\mathcal{L} \otimes \mathcal{G})\right),
$$

and the surjectivity of each of the factors follows from Lemmas 6.4 and 6.8. This completes the proof.

\section{The map $Q_{n, k}(E, \tau) \rightarrow B\left(S^{g} E, \sigma^{\prime}, \mathcal{L}_{n / k}^{\prime}\right)$ when the characteristic variety is $S^{g} E$}

Now we use the results in sections 5 and 6 when the characteristic variety for $Q_{n, k}(E, \tau)$ is $S^{g} E$ to show that $\Psi_{n / k}: Q_{n, k}(E, \tau) \rightarrow B\left(S^{g} E, \sigma^{\prime}, \mathcal{L}_{n / k}^{\prime}\right)$ is surjective and its kernel is generated in degree $\leq 3$. 


\subsection{Explicit description of $\sigma^{\prime}: X_{n / k} \rightarrow X_{n / k}$ when $X_{n / k} \cong S^{g} E$}

We write $\left[m, 2^{r}\right]$ and $\left[2^{r}, m\right]$ for the continued fractions $[m, 2, \ldots, 2]$ and $[2, \ldots, 2, m]$, respectively, when the number of twos is $r$.

Proposition 7.1. The characteristic variety $X_{n / k}$ is isomorphic to the gth symmetric power $S^{g} E$ if and only if $\frac{n}{k}$ is equal to either $\left[m, 2^{g-1}\right]$ or $\left[2^{g-1}, m\right]$ for some $m \geq 3$. In these cases,

(1) $\frac{n}{k}=\left[m, 2^{g-1}\right]$ if and only if $n=(m-1) g+1$ and $k=g$;

(2) $\frac{n}{k}=\left[2^{g-1}, m\right]$ if and only if $n=(m-1) g+1$ and $k=(m-1)(g-1)+1$;

(3) the morphism $\rho: E^{g} \rightarrow S^{g} E$ given by

$$
\rho\left(z_{1}, \ldots, z_{g}\right)= \begin{cases}\left(\left(z_{2}-z_{1}, z_{3}-z_{2}, \ldots, z_{g}-z_{g-1},-z_{g}\right)\right) & \text { when } \frac{n}{k}=\left[m, 2^{g-1}\right], \\ \left(\left(-z_{1}, z_{1}-z_{2}, z_{2}-z_{3}, \ldots, z_{g-1}-z_{g}\right)\right) & \text { when } \frac{n}{k}=\left[2^{g-1, m}\right]\end{cases}
$$

is a quotient for the action of $\Sigma_{n / k}$ on $E^{g}$.

Proof. Induction arguments on $g$ show that $\frac{(m-1) g+1}{g}=\left[m, 2^{g-1}\right]$ and $\frac{(m-1) g+1}{(m-1)(g-1)+1}=\left[2^{g-1}, m\right]$. It is easy to verify that $g$ and $(m-1)(g-1)+1$ are mutual inverses in $\mathbb{Z}_{n}$ when $n=(m-1) g+1$. By [CKS19b, Corollary 4.24], $E^{g} / \Sigma_{n / k}$ is isomorphic to $S^{g} E$ if and only if $\frac{n}{k}$ is equal to either $\left[m, 2^{g-1}\right.$ ] or $\left[2^{g-1}, m\right]$ for some $m \geq 3$; or equivalently, if and only if $\Sigma_{n / k} \cong \Sigma_{g}$ (though the action of $\Sigma_{g}$ on $E^{g}$ is not the 'natural' one). Part (3) is proved in [CKS19b, Proposition 4.25].

Proposition 7.2. Assume $g \geq 1$ and $m \geq 3$. Assume $\frac{n}{k}$ is either $\left[m, 2^{g-1}\right]$ or $\left[2^{g-1}, m\right]$. Let $\rho: E^{g} \rightarrow$ $S^{g} E$ be the corresponding quotient map in equation (7.1). Let $\tau^{\prime}=(m-2) \tau$. There is a commutative diagram

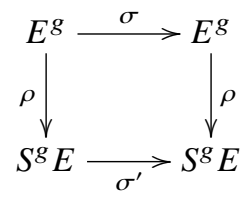

in which $\sigma: E^{g} \rightarrow E^{g}$ is the automorphism $\sigma\left(z_{1}, \ldots, z_{g}\right)=\left(z_{1}+\tau_{1}, \ldots, z_{g}+\tau_{g}\right)$ defined in \$3.1.4 and $\sigma^{\prime}: S^{g} E \rightarrow S^{g} E$ is the automorphism $\sigma^{\prime}\left(\left(z_{1}, \ldots, z_{g}\right)\right)=\left(\left(z_{1}+\tau^{\prime}, \ldots, z_{g}+\tau^{\prime}\right)\right)$.

Proof. In both cases, $n=(m-1) g+1$. By definition, $\tau_{i}=\left(k_{i}+l_{i}-n\right) \tau$, where $k_{i}$ and $l_{i}$ are the integers defined in $\$ 3.1 .4$. Since the characteristic variety $X_{n / k}$ is isomorphic to $E^{g} / \Sigma_{n / k}$, the existence and uniqueness of the automorphism $\sigma^{\prime}: S^{g} E \rightarrow S^{g} E$ making the diagram commute are established in [CKS19b, Proposition 2.10].

(1) If $\frac{n}{k}=\left[m, 2^{g-1}\right]$, then (3.2) implies

$$
m k_{1}=k_{0}+k_{2} \quad \text { and } \quad 2 k_{i}=k_{i-1}+k_{i+1} \quad(2 \leq i \leq g) .
$$

Since $k_{0}=n$ and $k_{1}=k$ as in (3.1),

$$
k_{g+1}-k_{g}=k_{g}-k_{g-1}=\cdots=k_{2}-k_{1}=(m-1) k_{1}-k_{0}=(m-1) k-n,
$$

which is, by Proposition 7.1(1), equal to -1 . Hence

$$
k_{i}=k_{1}+(i-1)(-1)=g-i+1
$$

for $i=1, \ldots, g+1$. Since $l_{0}=0, l_{1}=1$ and $l_{i}$ satisfy the same inductive formula as $k_{i}$,

$$
l_{g+1}-l_{g}=l_{g}-l_{g-1}=\cdots=l_{2}-l_{1}=(m-1) l_{1}-l_{0}=m-1 .
$$


Thus

$$
l_{i}=l_{1}+(i-1)(m-1)=(i-1)(m-1)+1 .
$$

It follows that $\tau_{i}=\left(k_{i}+l_{i}-n\right) \tau=(2-m)(g-i+1) \tau$ for $i=1, \ldots, g$. For all $i=1, \ldots, g-1$, $\tau_{i+1}-\tau_{i}=(m-2) \tau=\tau^{\prime}$. Therefore

$$
\begin{aligned}
\rho \sigma\left(z_{1}, \ldots, z_{g}\right) & =\rho\left(z_{1}+\tau_{1}, \ldots, z_{g}+\tau_{g}\right) \\
& =\left(\left(z_{2}+\tau_{2}-z_{1}-\tau_{1}, \ldots, z_{g}+\tau_{g}-z_{g-1}-\tau_{g-1},-z_{g}-\tau_{g}\right)\right) \\
& =\left(\left(z_{2}-z_{1}+\tau^{\prime}, \ldots, z_{g}-z_{g-1}+\tau^{\prime},-z_{g}+\tau^{\prime}\right)\right) \\
& =\sigma^{\prime}\left(\left(z_{2}-z_{1}, \ldots, z_{g}-z_{g-1},-z_{g}\right)\right) .
\end{aligned}
$$

Thus, $\rho \sigma=\sigma^{\prime} \rho$.

(2) Suppose $\frac{n}{k}=\left[2^{g-1}, m\right]$. Since

$$
2 k_{i}=k_{i-1}+k_{i+1} \quad(1 \leq i \leq g-1) \quad \text { and } \quad m k_{g}=k_{g-1}+k_{g+1},
$$

it follows that

$$
k_{1}-k_{0}=k_{2}-k_{1}=\cdots=k_{g}-k_{g-1}=k_{g+1}-(m-1) k_{g}=-(m-1) .
$$

Hence

$$
k_{i}=k-(i-1)(m-1)=(g-i)(m-1)+1
$$

for $i=1, \ldots, g$. By (3.1) and the proof of Proposition 7.1, $l_{g}=k^{\prime}=g$. Hence

$$
l_{1}-l_{0}=l_{2}-l_{1}=\cdots=l_{g}-l_{g-1}=l_{g+1}-(m-1) l_{g}=n-(m-1) k^{\prime}=1
$$

implies that $l_{i}=i$ for $i=1, \ldots, g$. It follows that $\tau_{i}=\left(k_{i}+l_{i}-n\right) \tau=-(m-2) i \tau$ for $i=1, \ldots, g$. For all $i=1, \ldots, g-1, \tau_{i}-\tau_{i+1}=(m-2) \tau$. Now,

$$
\begin{aligned}
\rho \sigma\left(z_{1}, \ldots, z_{g}\right) & =\rho\left(z_{1}+\tau_{1}, \ldots, z_{g}+\tau_{g}\right) \\
& =\left(\left(-z_{1}-\tau_{1}, z_{1}+\tau_{1}-z_{2}-\tau_{2}, \ldots, z_{g-1}+\tau_{g-1}-z_{g}-\tau_{g}\right)\right) \\
& =\left(\left(-z_{1}+\tau^{\prime}, z_{1}-z_{2}+\tau^{\prime}, \ldots, z_{g-1}-z_{g}+\tau^{\prime}\right)\right) .
\end{aligned}
$$

Thus, $\rho \sigma=\sigma^{\prime} \rho$.

\subsection{The special case $Q_{5,2}(E, \tau)$}

When $(n, k)=(5,2), \frac{n}{k}=[3,2]$, so $\sigma^{\prime}: S^{2} E \rightarrow S^{2} E$ is given by

$$
\sigma^{\prime}\left(\left(z_{1}, z_{2}\right)\right)=\left(\left(z_{1}+\tau, z_{2}+\tau\right)\right) .
$$

Hence Proposition 7.2 agrees with a remark after [FO89, Proposition 4.2] which says there is a (surjective) homomorphism $Q_{5,2}(E, \tau) \rightarrow B\left(S^{2} E, v, \mathcal{L}^{\prime}\right)$, where $v$ is the automorphism $\left(\left(z_{1}, z_{2}\right)\right) \mapsto$ $\left(\left(z_{1}+\tau, z_{2}+\tau\right)\right)$. The next result shows there is also a (surjective) homomorphism $Q_{5,2}(E, \tau) \rightarrow$ $B\left(S^{2} E, v^{-1}, \mathcal{L}^{\prime}\right)$, where $v^{-1}$ is the automorphism $\left(\left(z_{1}, z_{2}\right)\right) \mapsto\left(\left(z_{1}-\tau, z_{2}-\tau\right)\right)$.

Proposition 7.3. Let $m$ be an integer $\geq 3$ and assume $n=(m-1) g+1$ and $k=g$. Thus $X_{n / k} \cong S^{g} E$. Let $\sigma: E^{g} \rightarrow E^{g}$ be the automorphism that is translation by $\left(\tau_{1}, \ldots, \tau_{g}\right)$. Let $\sigma_{1}^{\prime}, \sigma_{2}^{\prime}: S^{g} E \rightarrow S^{g} E$ be the automorphisms such that $\rho_{i} \sigma=\sigma_{i}^{\prime} \rho_{i}$, where $\rho_{1}, \rho_{2}: E^{g} \rightarrow S^{g} E$ are the quotient morphisms

$$
\begin{aligned}
& \rho_{1}\left(z_{1}, \ldots, z_{g}\right)=\left(\left(z_{2}-z_{1}, \ldots, z_{g}-z_{g-1},-z_{g}\right)\right) \\
& \rho_{2}\left(z_{1}, \ldots, z_{g}\right)=\left(\left(z_{1}-z_{2}, \ldots, z_{g-1}-z_{g}, z_{g}\right)\right)
\end{aligned}
$$


for the action of $\Sigma_{n / k}$ on $E^{g}$. If $\mathcal{L}_{i}^{\prime}=\left(\rho_{i *} \mathcal{L}_{n / k}\right)^{\Sigma_{n / k}}$, then there is an isomorphism of triples $(f, u)$ : $\left(S^{g} E, \sigma_{1}^{\prime}, \mathcal{L}_{1}^{\prime}\right) \rightarrow\left(S^{g} E, \sigma_{2}^{\prime}, \mathcal{L}_{2}^{\prime}\right)$, where $f: S^{g} E \rightarrow S^{g} E$ is the automorphism

$$
f\left(\left(z_{1}, \ldots, z_{g}\right)\right)=\left(\left(-z_{1}, \ldots,-z_{g}\right)\right)
$$

and hence an isomorphism $B\left(S^{g} E, \sigma_{1}^{\prime}, \mathcal{L}_{1}^{\prime}\right) \cong B\left(S^{g} E, \sigma_{2}^{\prime}, \mathcal{L}_{2}^{\prime}\right)$.

Proof. Since

$$
f \sigma_{1}^{\prime} \rho_{1}=f \rho_{1} \sigma=\rho_{2} \sigma=\sigma_{2}^{\prime} \rho_{2}=\sigma_{2}^{\prime} f \rho_{1}
$$

and $\rho_{1}$ is surjective, $f \sigma_{1}^{\prime}=\sigma_{2}^{\prime} f$. The proof will be complete once we show that $f^{*} \mathcal{L}_{2}^{\prime} \cong \mathcal{L}_{1}^{\prime}$.

Since $f$ is $\Sigma_{n / k}$-equivariant,

$$
f^{*} \mathcal{L}_{2}^{\prime}=f^{*}\left(\left(\rho_{2 *} \mathcal{L}_{n / k}\right)^{\Sigma_{n / k}}\right) \cong f_{*}^{-1}\left(\left(\rho_{2 *} \mathcal{L}_{n / k}\right)^{\Sigma_{n / k}}\right) \cong\left(f_{*}^{-1} \rho_{2 *} \mathcal{L}_{n / k}\right)^{\Sigma_{n / k}}=\left(\rho_{1 *} \mathcal{L}_{n / k}\right)^{\Sigma_{n / k}}=\mathcal{L}_{1}^{\prime}
$$

\subsection{The map $\Psi_{n / k}: Q_{n, k}(E, \tau) \rightarrow B\left(S^{g} E, \sigma^{\prime}, \mathcal{L}_{n / k}^{\prime}\right)$}

We continue to assume that $X_{n / k} \cong S^{g} E$ - that is, that $\frac{n}{k}$ is either $\left[m, 2^{g-1}\right]$ or $\left[2^{g-1}, m\right]$, where $m$ is an integer $\geq 3$. We identify $X_{n / k}$ with $S^{g} E$ via the quotient morphism $\rho: E^{g} \rightarrow S^{g} E$ in equation (7.1). In other words, there is a closed immersion $i$ such that $\Phi_{n / k}: E^{g} \rightarrow \mathbb{P}^{n-1}$ factors as

$$
E^{g} \stackrel{\rho}{\longrightarrow} S^{g} E \stackrel{i}{\longrightarrow} \mathbb{P}^{n-1}
$$

Let $\mathcal{L}_{n / k}^{\prime}=i^{*} \mathcal{O}_{\mathbb{P}^{n-1}}(1)$.

Lemma 7.4. Assume $\frac{n}{k}=\left[m, 2^{g-1}\right]$. Then $\left[\mathcal{L}_{n / k}^{\prime}\right]=D+(m-1) F$ in $\mathrm{NS}\left(S^{g} E\right)$.

Proof. By definition, $D=\left[D_{0}\right]$ and $F=\left[F_{0}\right]$, where $F_{0}=\left\{\left(\left(z_{1}, z_{2}, \ldots, z_{g}\right)\right) \mid z_{1}+\cdots+z_{g}=0\right\}$ and $D_{0}=\left\{\left(\left(0, z_{2}, \ldots, z_{g}\right)\right) \mid z_{2}, \ldots, z_{g} \in E\right\}$.

The Néron-Severi class of $\mathcal{L}_{n / k}^{\prime}$ is $a D+b F$ for some $a, b \in \mathbb{Z}$. The divisor $\rho^{*}\left(a D_{0}+b F_{0}\right)$ equals

$$
D^{\prime}:=a\left(\Delta_{1,2}+\cdots+\Delta_{g-1, g}+\left(E^{g-1} \times\{0\}\right)\right)+b\left(\{0\} \times E^{g-1}\right) .
$$

Since $\rho^{*} i^{*} \mathcal{O}_{\mathbb{P}^{n-1}}(1)=\Phi_{n / k}^{*} \mathcal{O}_{\mathbb{P}^{n-1}}(1)=\mathcal{O}_{E^{g}}\left(D_{n / k}\right)$, the divisors $D_{n / k}$ and $D^{\prime}$ are linearly equivalent and therefore give the same class in $\operatorname{NS}\left(E^{g}\right)$.

Fix a point $p \in E^{g-1}$ in general position and let $C_{1}$ and $C_{g}$ be the curves $E \times\{p\}$ and $\{p\} \times E$ on $S^{g} E$. Then $D^{\prime} \cdot C_{1}=a+b$ and $D_{n / k} \cdot C_{1}=(m-1)+1$, so $a+b=m$. Also, $D^{\prime} \cdot C_{2}=2 a$ and $D_{n / k} \cdot C_{2}=\left(n_{g}-1\right)+1=2$, so $2 a=2$. Therefore $a=1$ and $b=m-1$.

Theorem 7.5. Assume $\frac{n}{k}=\left[m, 2^{g-1}\right]$. For all translation automorphisms $\sigma: S^{g} E \rightarrow S^{g} E$, the algebra $B\left(S^{g} E, \sigma, \mathcal{L}_{n / k}^{\prime}\right)$ is generated in degree one and has relations in degrees 2 and 3.

Proof. By Lemma 7.4, $\left[\mathcal{L}_{n / k}^{\prime}\right]=D+(m-1) F$. Since $m \geq 3$, Theorem 6.9 applies.

Corollary 7.6. Fix an integer $m \geq 3$ and assume $\frac{n}{k}=\left[m, 2^{g-1}\right]$. Let $\sigma^{\prime}: S^{g} E \rightarrow S^{g} E$ be the translation automorphism by $(m-2) \tau$-that is, the automorphism in Proposition 7.2. The homomorphism $\Psi_{n / k}: Q_{n, k}(E, \tau) \rightarrow B\left(S^{g} E, \sigma^{\prime}, \mathcal{L}_{n / k}^{\prime}\right)$ is surjective and $\operatorname{ker}\left(\Psi_{n / k}\right)$ is generated by elements of degree $\leq 3$.

Proof. This follows immediately from Theorem 7.5. 


\subsection{The algebras $Q_{2 k+1, k}(E, \tau)$}

Since $\frac{2 k+1}{k}=\left[3,2^{k-1}\right], X_{(2 k+1) / k}=S^{k} E \subseteq \mathbb{P}^{2 k}$.

Lemma 7.7. If $\tau \in(E-E[2]) \cup\{0\}$, then the kernel of the homomorphism $\Psi_{(2 k+1) / k}: Q_{2 k+1, k}(E, \tau) \rightarrow$ $B\left(S^{k} E, \sigma^{\prime}, \mathcal{L}_{(2 k+1) / k}^{\prime}\right)$ is generated by $\frac{1}{6} k(k+1)(2 k+1)$ elements of degree 3.

Proof. For brevity, we write $\Psi=\Psi_{(2 k+1) / k}$ and $\mathcal{L}^{\prime}=\mathcal{L}_{(2 k+1) / k}^{\prime}$.

Let $Q_{j}$ and $B_{j}$ denote the degree- $j$ components of $Q_{2 k+1, k}(E, \tau)$ and $B\left(S^{k} E, \sigma^{\prime}, \mathcal{L}^{\prime}\right)$, respectively.

To prove that $\operatorname{ker}(\Psi)$ is zero in degree two, we must show that $\operatorname{dim}\left(Q_{2}\right)=\operatorname{dim}\left(B_{2}\right)$. Since $\tau \in$ $(E-E[2]) \cup\{0\}$, we see in $[\mathrm{CKS} 20$, Theorem 5.10] that

$$
\operatorname{dim}\left(Q_{2}\right)=\left(\begin{array}{c}
2 k+2 \\
2
\end{array}\right)=(2 k+1)(k+1) .
$$

We will use a special case of [CC93, Theorem 1.17]: let $\mathcal{L}$ be an invertible sheaf on $S^{k} E$ such that $[\mathcal{L}]=a D+b F$; if $a \geq 0$ and $a+k b>0$, then

$$
\operatorname{dim} H^{0}\left(S^{k} E, \mathcal{L}\right)=\frac{(a+k b)}{k !} \prod_{i=1}^{k-1}(a+i) .
$$

Since $\left[\mathcal{L}^{\prime}\right]=D+2 F,\left[\mathcal{L}^{\prime} \otimes\left(\sigma^{\prime}\right)^{*} \mathcal{L}^{\prime}\right]=2 D+4 F$. Hence

$$
\operatorname{dim}\left(B_{2}\right)=\operatorname{dim} H^{0}\left(S^{k} E, \mathcal{L}^{\prime} \otimes\left(\sigma^{\prime}\right)^{*} \mathcal{L}^{\prime}\right)=\frac{(2+4 k)}{k !} \prod_{i=1}^{k-1}(2+i)=(1+2 k)(k+1) .
$$

Thus, $\operatorname{dim}\left(B_{2}\right)=\operatorname{dim}\left(Q_{2}\right)$.

On the other hand, $\operatorname{dim}\left(Q_{3}\right)=\left(\begin{array}{c}2 k+3 \\ 3\end{array}\right)$ and $\operatorname{dim}\left(B_{3}\right)$ is

$$
\operatorname{dim} H^{0}\left(S^{k} E, \mathcal{L}^{\prime} \otimes\left(\sigma^{\prime}\right)^{*} \mathcal{L}^{\prime} \otimes\left(\sigma^{\prime}\right)^{2 *} \mathcal{L}^{\prime}\right)=\frac{(3+6 k)}{k !} \prod_{i=1}^{k-1}(3+i)=\frac{1}{2}(1+2 k)(k+1)(k+2),
$$

so $\operatorname{dim}\left(Q_{3}\right)-\operatorname{dim}\left(B_{3}\right)=\frac{1}{6} k(k+1)(2 k+1)$.

For example, the kernel of the map $Q_{5,2}(E, \tau) \rightarrow B\left(S^{2} E, \sigma^{\prime}, \mathcal{L}^{\prime}\right)$ is generated by five elements of degree 3 when $\tau \in(E-E[2]) \cup\{0\}$. When $\tau=0$ this recovers the well-known fact that the image of the map $S^{2} E \rightarrow \mathbb{P}^{4}$ is the intersection of five cubic hypersurfaces. Feigin and Odesskii say that the subalgebra of $Q_{5,2}(E, \tau)$ generated by those five degree-3 elements is isomorphic to $Q_{5,1}(E, \tau)$ [FO89, p. 25]. We do not know how to prove this.

Proposition 7.8. Let $\mathbb{P}^{2 k}=\mathbb{P}\left(V^{*}\right)$ and let $i: S^{k} E \rightarrow \mathbb{P}^{2 k}$ be the closed immersion given by the complete linear system $\left|\mathcal{L}^{\prime}\right|$, where $\left[\mathcal{L}^{\prime}\right]=D+2 F$. If $\tau \in(E-E[2]) \cup\{0\}$, then the space of relations for $Q_{2 k+1, k}(E, \tau)$ is the subspace of $V \otimes V$ vanishing on the graph of the automorphism $\sigma^{\prime}: S^{k} E \rightarrow S^{k} E$ that is translation by $\tau$.

Proof. As in Lemma 7.7, we write $\Psi=\Psi_{(2 k+1) / k}$ and $\mathcal{L}^{\prime}=\mathcal{L}_{(2 k+1) / k}^{\prime}$.

Because $\Psi$ is an isomorphism in degree two, $Q_{2 k+1, k}(E, \tau)$ and $B\left(S^{k} E, \sigma^{\prime}, \mathcal{L}^{\prime}\right)$ have the same quadratic relations. Thus, the space of quadratic relations for $Q_{2 k+1, k}(E, \tau)$ coincides with the kernel of the multiplication map

$$
B_{1} \otimes B_{1}=H^{0}\left(S^{k} E \times S^{k} E, \mathcal{L}^{\prime} \otimes \mathcal{L}^{\prime}\right) \longrightarrow H^{0}\left(S^{k} E, \mathcal{L}^{\prime} \otimes\left(\sigma^{\prime}\right)^{*} \mathcal{L}^{\prime}\right)=B_{2} .
$$

Let $X=S^{k} E$ and let $\Gamma_{\sigma^{\prime}} \subseteq X^{2}$ denote the graph of $\sigma^{\prime}$. If we apply the functor $\left(\mathcal{L}^{\prime} \otimes \mathcal{L}^{\prime}\right) \otimes_{\mathcal{O}_{X^{2}}}-$ to the exact sequence $0 \rightarrow \mathcal{O}_{X^{2}}\left(-\Gamma_{\sigma^{\prime}}\right) \rightarrow \mathcal{O}_{X^{2}} \rightarrow \mathcal{O}_{\Gamma_{\sigma^{\prime}}} \rightarrow 0$ and take global sections, it becomes clear that 
the kernel is $H^{0}\left(X^{2},\left(\mathcal{L}^{\prime} \otimes \mathcal{L}^{\prime}\right)\left(-\Gamma_{\sigma^{\prime}}\right)\right)$, which is the subspace of $H^{0}\left(S^{k} E \times S^{k} E, \mathcal{L}^{\prime} \otimes \mathcal{L}^{\prime}\right)$ consisting of the sections that vanish on $\Gamma_{\sigma^{\prime}}$.

Since $\frac{2 k+1}{k}=\left[3,2^{k-1}\right]$, Proposition 7.2 tells us that $\sigma^{\prime}\left(\left(x_{1}, \ldots, x_{k}\right)\right)=\left(\left(x_{1}+\tau, \ldots, x_{k}+\tau\right)\right)$.

\section{The rings $B\left(E^{g}, \sigma, \mathcal{L}_{n / k}\right)$}

In this and the next section we assume that the following equivalent conditions hold:

(1) $X_{n / k}=E^{g}$.

(2) $\mathcal{L}_{n / k}$ is very ample.

(3) All $n_{i}$ in $\left[n_{1}, \ldots, n_{g}\right]=\frac{n}{k}$ are $\geq 3$.

Let $\sigma: E^{g} \rightarrow E^{g}$ be an arbitrary translation automorphism.

In this section we show that $B\left(E^{g}, \sigma, \mathcal{L}_{n / k}\right)$ is generated in degree one.

In section 9 we show that the ideal of relations for $B\left(E^{g}, \sigma, \mathcal{L}_{n / k}\right)$ is generated by elements of degree $\leq 3$. Finally, we apply this to the particular $\sigma$ relevant to $Q_{n, k}(E, \tau)$.

\subsection{The main result in this section}

The fact that $B\left(E^{g}, \sigma, \mathcal{L}_{n / k}\right)$ is generated in degree one will follow from Proposition 8.1, the proof of which occupies a significant portion of this section.

Proposition 8.1. Suppose all $n_{i}$ in $\left[n_{1}, \ldots, n_{g}\right]=\frac{n}{k}$ are $\geq 3$. If $\mathcal{L}^{\prime}$ and $\mathcal{L}^{\prime \prime}$ are tensor products of translates of $\mathcal{L}_{n / k}$, then the multiplication map

$$
H^{0}\left(E^{g}, \mathcal{L}^{\prime}\right) \otimes H^{0}\left(E^{g}, \mathcal{L}^{\prime \prime}\right) \longrightarrow H^{0}\left(E^{g}, \mathcal{L}^{\prime} \otimes \mathcal{L}^{\prime \prime}\right)
$$

is onto.

Our strategy for proving Proposition 8.1 is similar to that used to prove Proposition 5.6(2).

\subsection{Notation}

Most of the notation in this section and the next is the same as in $\$ 3.1 .6$, though we make some simplifications and introduce some new notation as follows:

(1) $X=E^{g}$.

(2) $\pi: E^{g} \rightarrow E$ is the projection $\pi\left(z_{1}, \ldots, z_{g}\right)=z_{g}$.

(3) $\pi^{\prime}: E^{g} \rightarrow E^{g-1}$ is the projection $\pi\left(z_{1}, \ldots, z_{g}\right)=\left(z_{1}, \ldots, z_{g-1}\right)$.

(4) $\rho: E^{g-1} \rightarrow E$ is the projection $\pi\left(z_{1}, \ldots, z_{g-1}\right)=z_{g-1}$.

(5) $\mathcal{L}=\mathcal{L}_{n / k}$.

(6) $D=D_{n / k}$ defined in (3.4); thus $\mathcal{L}=\mathcal{O}_{E^{g}}(D)$.

(7) $\sigma: E^{g} \rightarrow E^{g}$ is an arbitrary translation automorphism.

\subsection{Preliminary results}

Proposition 8.2. Let $k^{\prime}$ be the unique integer such that $n>k^{\prime} \geq 1$ and $k k^{\prime}=1(\bmod n)$.

(1) $\pi_{*} \mathcal{L}$ is a locally free $\mathcal{O}_{E}$-module of degree $n$ and rank $k^{\prime}$.

(2) $\mu\left(\pi_{*} \mathcal{L}\right)=n / k^{\prime}$.

(3) $H^{1}\left(E, \pi_{*} \mathcal{L}\right)=0$.

Proof. For all $z \in E$, the restriction $\mathcal{L}_{z}$ of $\mathcal{L}$ to $X_{z}=\pi^{-1}(z)$ is a standard divisor of type $\left(n_{1}, \ldots, n_{g-1}\right)$ (see $\$ 3.1 .6$ ), which is very ample because all $n_{i}$ are $\geq 3$. The dimension of $H^{0}\left(\mathcal{L}_{z}\right)$ is $d\left(n_{1}, \ldots, n_{g-1}\right)$ (see 
$\S 3.1 .4)$, and $H^{q}\left(\mathcal{L}_{\left[n_{1}, \ldots, n_{g-1}\right]}\right)=0$ for all $q \geq 1$. Since the dimension of $H^{0}\left(X_{z}, \mathcal{L}_{z}\right)$ is independent of $z$, Grauert's theorem [Har77, Corollary III.12.9] tells us that $\pi_{*} \mathcal{L}$ is locally free of rank $d\left(n_{1}, \ldots, n_{g-1}\right)$. The higher cohomology groups of $\mathcal{L}_{z}$ are zero, so by Grauert's Theorem again, the higher cohomology groups of $\pi_{*} \mathcal{L}$ are also zero.

Since $H^{1}\left(E, \pi_{*} \mathcal{L}\right)=0$, the dimension of $H^{0}\left(E, \pi_{*} \mathcal{L}\right) \cong H^{0}(X, \mathcal{L})$ equals the degree of $\pi_{*} \mathcal{L}$. Hence

$$
\mu\left(\pi_{*} \mathcal{L}\right)=\frac{d\left(n_{1}, \ldots, n_{g}\right)}{d\left(n_{1}, \ldots, n_{g-1}\right)}=\left[n_{g}, \ldots, n_{1}\right]=\frac{n}{k^{\prime}} .
$$

The proof is complete.

Lemma 8.3. For all $x \in E^{g}, \pi_{*} T_{x}^{*} \mathcal{L}$ is indecomposable of slope $>2$.

Proof. Since $\pi_{*} T_{x}^{*} \mathcal{L}=T_{x_{g}}^{*} \pi_{*} \mathcal{L}$, we can assume $x=0$. The slope of $\pi_{*} \mathcal{L}$ is $n / k^{\prime}$ by Proposition 8.2. Using the fact that all $n_{i}$ are $\geq 3$, an induction argument on $g$ shows that $n / k^{\prime}>2$.

We prove that $\pi_{*} \mathcal{L}$ is indecomposable by induction on $g$. The case $g=1$ is trivial, so we assume that $g \geq 2$ and that the result is true for $g-1$. The induction hypothesis will be applied to the left-hand factor in $E^{g-1} \times E$ and the sheaf $\mathcal{L}_{\left[n_{1}, \ldots, n_{g-1}+1\right]}$.

In [CKS19b, §3.1.3], we observed that $D=D_{n / k}$ is linearly equivalent to

$$
D_{\left[n_{1}, \ldots, n_{g}\right]}^{\prime}=\sum_{i=1}^{g} E^{i-1} \times \mathfrak{D}_{i}^{\prime} \times E^{g-i}-\sum_{j=1}^{g-1} \Delta_{j, j+1}^{\prime},
$$

where $\mathfrak{b}_{i}^{\prime}=\left(n_{i}+2-\delta_{i, 1}-\delta_{i, g}\right)(0)$ and

$$
\Delta_{j, j+1}^{\prime}=\left\{\left(z_{1}, \ldots, z_{g}\right) \in E^{g} \mid z_{j}+z_{j+1}=0\right\} .
$$

Thus $\mathcal{L} \cong \mathcal{O}_{E^{g}}\left(D_{\left[n_{1}, \ldots, n_{g}\right]}^{\prime}\right)$. The rest of the proof uses the divisor $D_{\left[n_{1}, \ldots, n_{g}\right]}^{\prime}$ rather than $D_{n / k}$. Let

$$
D^{\prime \prime}=D_{\left[n_{1}, \ldots, n_{g}\right]}^{\prime}-E^{g-1} \times \mathfrak{D}_{g}^{\prime}
$$

Corresponding to the factorisation $E^{g}=E^{g-1} \times E$ and the decomposition $D=D^{\prime \prime}+\left(E^{g-1} \times \mathfrak{D}_{g}^{\prime}\right)$,

$$
\mathcal{L} \cong \mathcal{M} \otimes \pi^{*} \mathcal{F}
$$

where $\mathcal{M}:=\mathcal{O}_{E^{g}}\left(D^{\prime \prime}\right)$ and $\mathcal{F}:=\mathcal{O}_{E}\left(\mathfrak{D}_{g}^{\prime}\right)$. By the Projection Formula,

$$
\pi_{*} \mathcal{L} \cong \pi_{*} \mathcal{M} \otimes \mathcal{F}
$$

Since $\mathcal{F}$ is an invertible $\mathcal{O}_{E}$-module, $\pi_{*} \mathcal{L}$ is indecomposable if and only if $\pi_{*} \mathcal{M}$ is. We will show that $\pi_{*} \mathcal{M}$ is indecomposable.

By Grauert's theorem [Har77, Corollary III.12.9], $\pi_{*} \mathcal{M}$ is the locally free $\mathcal{O}_{E}$-module whose fibre at $z \in E$ is

$$
\left(\pi_{*} \mathcal{M}\right)_{z} \cong H^{0}\left(E^{g-1}, \mathcal{O}\left(D_{L}^{\prime}-E^{g-2} \times\{-z\}\right)\right),
$$

where $D_{L}^{\prime}:=D_{\left[n_{1}, \ldots, n_{g-1}+1\right]}^{\prime}$ and $E^{g-1}$ is identified with $E^{g-1} \times\{z\} \subseteq E^{g}$. The inclusion $\mathcal{O}_{E^{g-1}}\left(D_{L}^{\prime}-\right.$ $\left.E^{g-2} \times\{-z\}\right) \subseteq \mathcal{O}_{E^{g-1}}\left(D_{L}^{\prime}\right)$ gives rise to an inclusion

$$
\left(\pi_{*} \mathcal{M}\right)_{z} \cong H^{0}\left(E^{g-1}, \mathcal{O}\left(D_{L}^{\prime}-E^{g-2} \times\{-z\}\right)\right) \subseteq H^{0}\left(E^{g-1}, \mathcal{O}\left(D_{L}^{\prime}\right)\right)
$$

at the level of global sections. This inclusion is the $z$-fibre of a monomorphism

$$
\pi_{*} \mathcal{M} \longrightarrow H^{0}\left(E^{g-1}, \mathcal{O}\left(D_{L}^{\prime}\right)\right) \otimes \mathcal{O}_{E}
$$


between locally free $\mathcal{O}_{E}$-modules. The cokernel of the monomorphism in (8.3) is $\theta^{*} \rho_{*} \mathcal{O}_{E^{g-1}}\left(D_{L}^{\prime}\right)$, where $\theta$ is the involution $z \mapsto-z$ on $E$. After tracing through the foregoing identification, the resulting map

$$
\begin{aligned}
H^{0}\left(E^{g-1}, \mathcal{O}\left(D_{L}^{\prime}\right)\right) \otimes \mathcal{O}_{E} & \cong H^{0}\left(E, \rho_{*} \mathcal{O}\left(D_{L}^{\prime}\right)\right) \otimes \mathcal{O}_{E} \\
& \cong H^{0}\left(E, \theta^{*} \rho_{*} \mathcal{O}\left(D_{L}^{\prime}\right)\right) \otimes \mathcal{O}_{E} \\
& \rightarrow \theta^{*} \rho_{*} \mathcal{O}\left(D_{L}^{\prime}\right)
\end{aligned}
$$

is the canonical surjection exhibiting the right-hand side as a locally free sheaf that is generated by its global sections.

This means that $\pi_{*} \mathcal{M}$ can be identified with the shift $T_{\mathcal{O}}\left(\theta^{*} \rho_{*} \mathcal{O}\left(D_{L}^{\prime}\right)\right)[-1]$, where $T_{\mathcal{O}}$ is the autoequivalence of the bounded derived category $\mathrm{D}^{b}(\operatorname{coh}(E))$ from $\$ 4$.3.3. By the induction hypothesis, $\theta^{*} \rho_{*} \mathcal{O}\left(D_{L}^{\prime}\right) \cong \theta^{*} \rho_{*} \mathcal{L}_{\left[n_{1}, \ldots, n_{g-1}+1\right]}$ is indecomposable, so $\pi_{*} \mathcal{M}$ is also indecomposable.

\subsubsection{Remark}

The exact sequence

$$
0 \longrightarrow \pi_{*} \mathcal{M} \longrightarrow H^{0}\left(E^{g-1}, \mathcal{O}\left(D_{L}^{\prime}\right)\right) \otimes \mathcal{O}_{E} \longrightarrow \theta^{*} \rho_{*}\left(\mathcal{O}\left(D_{L}^{\prime}\right)\right) \longrightarrow 0
$$

that appeared in the proof of Lemma 8.3 can be obtained in another way. First, for simplicity, write $\Delta^{\prime}$ for $\Delta_{g-1, g}^{\prime}$, and let $j: \Delta^{\prime} \rightarrow X$ be the inclusion. After applying the functor $\mathcal{O}_{X}\left(D_{L}^{\prime} \times E\right) \otimes-$ to

$$
0 \rightarrow \mathcal{O}_{X}\left(-\Delta^{\prime}\right) \rightarrow \mathcal{O}_{X} \rightarrow j_{*} \mathcal{O}_{\Delta^{\prime}} \rightarrow 0
$$

we obtain

$$
0 \rightarrow \mathcal{M} \rightarrow \mathcal{O}_{X}\left(D_{L}^{\prime} \times E\right) \rightarrow\left(j_{*} \mathcal{O}_{\Delta^{\prime}}\right)\left(D_{L}^{\prime} \times E\right) \rightarrow 0 .
$$

Here $\mathcal{O}_{X}\left(D_{L}^{\prime} \times E\right)=\mathcal{O}_{E^{g-1}}\left(D_{L}^{\prime}\right) \otimes \mathcal{O}_{E}$ and

$$
\begin{aligned}
\left(j_{*} \mathcal{O}_{\Delta^{\prime}}\right)\left(D_{L}^{\prime} \times E\right) & \cong\left(j_{*} \mathcal{O}_{\Delta^{\prime}}\right) \otimes \mathcal{O}_{X}\left(D_{L}^{\prime} \times E\right) \\
& \cong j_{*}\left(\mathcal{O}_{\Delta^{\prime}} \otimes j^{*} \mathcal{O}_{X}\left(D_{L}^{\prime} \times E\right)\right) \\
& \cong j_{*} j^{*} \pi^{\prime *}\left(\mathcal{O}_{E^{g-1}}\left(D_{L}^{\prime}\right)\right)
\end{aligned}
$$

by the Projection Formula. Grauert's theorem shows that the fibre of $R^{1} \pi_{*}(\mathcal{M})$ at $z \in E$ is isomorphic to

$$
H^{1}\left(X_{z}, \mathcal{M}_{z}\right) \cong H^{1}\left(E^{g-1}, \mathcal{O}_{E^{g-1}}\left(D_{L}^{\prime}-E^{g-2} \times\{-z\}\right)\right),
$$

which is zero by Proposition 3.1(5). Thus applying $\pi_{*}$ to the sequence in (8.4) produces the exact sequence

$$
0 \rightarrow \pi_{*} \mathcal{M} \rightarrow H^{0}\left(E^{g-1}, \mathcal{O}_{E^{g-1}}\left(D_{L}^{\prime}\right)\right) \otimes \mathcal{O}_{E} \rightarrow \pi_{*} j_{*} j^{*} \pi^{* *}\left(\mathcal{O}_{E^{g-1}}\left(D_{L}^{\prime}\right)\right) \rightarrow 0 .
$$

Since $\pi_{*} j_{*} j^{*} \pi^{\prime *}=\theta^{*} \rho_{*}$, this is the desired exact sequence.

Corollary 8.4. For all $x \in E^{g}, \pi_{*} T_{x}^{*} \mathcal{L}$ is stable of slope $>2$.

Proof. By Lemma 8.3, $\pi_{*} T_{x}^{*} \mathcal{L}$ is indecomposable of slope $>2$. Stability now follows from Lemma 4.5, together with the observation made in the course of the proof of Proposition 8.2 that the degree and rank of $\pi_{*} \mathcal{L}$ are $n$ and $k^{\prime}$, respectively, which are coprime.

To prove Proposition 8.1 we must deal with tensor products of translates of $\mathcal{L}$. A first step is the following observation:

Lemma 8.5. If $\mathcal{L}_{1}, \ldots, \mathcal{L}_{m}$ are translates of $\mathcal{L}$, then $\mu\left(\pi_{*}\left(\mathcal{L}_{1} \otimes \cdots \otimes \mathcal{L}_{m}\right)\right)=m \mu\left(\pi_{*} \mathcal{L}\right)$. 
Proof. In order to lighten the notation we address only the case $\mathcal{L}_{i}=\mathcal{L}$ for all $i$. The general case is analogous.

Since $\mathcal{L}=\mathcal{O}_{E^{g}}(D)$,

$$
\operatorname{deg}\left(\pi_{*} \mathcal{L}^{\otimes m}\right)=h^{0}\left(\pi_{*} \mathcal{L}^{\otimes m}\right)=h^{0}\left(\mathcal{L}^{\otimes m}\right)=\frac{(m D)^{g}}{g !}=m^{g} \operatorname{deg}\left(\pi_{*} \mathcal{L}\right),
$$

where the third equality follows from the Riemann-Roch theorem for abelian varieties [Mum08, III.16].

On the other hand, a similar argument to the proof of Lemma 8.3 shows that $\pi_{*} \mathcal{L}^{\otimes m}$ is a locally free $\mathcal{O}_{E}$-module whose fibre over $z \in E$ is

$$
H^{0}\left(E^{g-1}, \mathcal{O}_{E^{g-1}}\left(m \sum_{i=1}^{g-1} E^{i-1} \times D_{i} \times E^{g-i-1}+m E^{g-2} \times\{z\}+m \sum_{j=1}^{g-2} \Delta_{j, j+1}\right)\right) .
$$

Because $E^{g-2} \times D_{g-1}$ is the pullback of a degree- $\left(n_{g-1}-2+\delta_{g-1,1}\right)$ divisor on the rightmost factor $E$ of $E^{g-1}$, the parenthetic divisor in (8.5) is $m D^{\prime \prime}$ for a standard divisor $D^{\prime \prime}$ of type $\left(n_{1}, \ldots, n_{g-1}\right)$. By another application of the Riemann-Roch theorem, the dimension of the vector space in (8.5) is

$$
\frac{\left(m D^{\prime \prime}\right)^{g-1}}{(g-1) !}=m^{g-1} d\left(n_{1}, \cdots, n_{g-1}\right)=m^{g-1} \operatorname{rank}\left(\pi_{*} \mathcal{L}\right) .
$$

In conclusion, lifting $\mathcal{L}$ to the $m$ th tensor power scales the degree of $\pi_{*} \mathcal{L}$ by $m^{g}$ and its rank by $m^{g-1}$. In conclusion, the slope scales by $m$, as claimed.

\subsubsection{Remark}

Lemma 8.5 says that the map $\mu\left(\pi_{*}-\right)$ is a morphism from the subsemigroup of $\mathrm{NS}\left(E^{g}\right)$ generated by $\mathcal{L}$ to $\mathbb{Q}>0$.

\subsection{Proof of Proposition 8.1}

We will prove the following more precise version of Proposition 8.1:

Lemma 8.6. If $\mathcal{L}_{0}, \ldots, \mathcal{L}_{m}$ are translates of $\mathcal{L}$, then each $\pi_{*}\left(\mathcal{L}_{0} \otimes \cdots \otimes \mathcal{L}_{i}\right)$ is semistable and the map

$$
H^{0}\left(E^{g}, \mathcal{L}_{0}\right) \otimes H^{0}\left(E^{g}, \mathcal{L}_{1} \otimes \cdots \otimes \mathcal{L}_{m}\right) \longrightarrow H^{0}\left(E^{g}, \mathcal{L}_{0} \otimes \cdots \otimes \mathcal{L}_{m}\right)
$$

is onto.

Proof. We denote the statement in the lemma by $\mathbf{P}_{g, m}$, since it depends on both indices. We will argue by induction on $g+m$. To make sure that the induction works, we prove $\mathbf{P}_{g, m}$ when $\mathcal{L}$ is an arbitrary standard divisor of type $\left[n_{1}, \ldots, n_{g}\right]$. However, for simplicity, we write the proof only for $\mathcal{L}=\mathcal{L}_{n / k}$.

The case $m=0$ is straightforward, and the case $g=1$ follows from Corollary 4.11. From now on we assume that $g \geq 2$ and $m \geq 1$.

We assume without loss of generality, as will be clear from the proof, that all $\mathcal{L}_{i}$ are isomorphic to $\mathcal{L}$.

Identifying, for the locally free sheaves in question, $H^{0}\left(E^{g},-\right)$ with $H^{0}\left(E, \pi_{*}(-)\right)$, the surjectivity claim decomposes into the two separate demands that

$$
H^{0}\left(E, \pi_{*} \mathcal{L}\right) \otimes H^{0}\left(E, \pi_{*} \mathcal{L}^{\otimes m}\right) \rightarrow H^{0}\left(E, \pi_{*} \mathcal{L} \otimes \pi_{*} \mathcal{L}^{\otimes m}\right)
$$

and

$$
H^{0}\left(E, \pi_{*} \mathcal{L} \otimes \pi_{*} \mathcal{L}^{\otimes m}\right) \rightarrow H^{0}\left(E, \pi_{*} \mathcal{L}^{\otimes(m+1)}\right)
$$

both be onto. 
The semistability claim in $\mathbf{P}_{g, m-1}$ implies that the two locally free sheaves appearing in the domain of the map in (8.6) are semistable. By Lemmas 8.3 and $8.5, \pi_{*} \mathcal{L}$ and $\pi_{*} \mathcal{L}^{\otimes m}$ have slope $>2$, so Corollary 4.11 tells us that the map in (8.6) is surjective.

It remains to show that the map in (8.7) is onto. As before, let $z \in E$ and define $X_{z}=\pi^{-1}(z)=$ $E^{g-1} \times\{z\} \subseteq E^{g}$. By Grauert's theorem, for $z \in E$ the $z$-fibre of the map

$$
\pi_{*} \mathcal{L} \otimes \pi_{*} \mathcal{L}^{\otimes m} \longrightarrow \pi_{*} \mathcal{L}^{\otimes(m+1)}
$$

is

$$
H^{0}\left(X_{z},\left.\mathcal{L}\right|_{X_{z}}\right) \otimes H^{0}\left(X_{z},\left.\mathcal{L}^{\otimes m}\right|_{X_{z}}\right) \longrightarrow H^{0}\left(X_{z},\left.\mathcal{L}^{\otimes(m+1)}\right|_{X_{z}}\right)
$$

The locally free sheaf $\left.\mathcal{L}\right|_{X_{z}}$ on $X_{z} \cong E^{g-1}$ is a standard divisor of type $\left(n_{1}, \ldots, n_{g-1}\right)$. The induction hypothesis $\mathbf{P}_{g-1, m}$ shows that the map in (8.9) is onto, and hence the map in (8.8) is an epimorphism. The domain of the map in (8.8) is semistable by $\mathbf{P}_{g, m-1}$ and Lemma 4.6, and the slopes of $\pi_{*} \mathcal{L} \otimes \pi_{*} \mathcal{L}^{\otimes m}$ and $\pi_{*} \mathcal{L}^{\otimes(m+1)}$ are the same by Lemma 8.5. It follows that the three terms in the exact sequence

$$
0 \rightarrow \mathcal{K} \rightarrow \pi_{*} \mathcal{L} \otimes \pi_{*} \mathcal{L}^{\otimes m} \rightarrow \pi_{*} \mathcal{L}^{\otimes(m+1)} \rightarrow 0
$$

are all semistable and of equal (positive) slopes. $\operatorname{Since} \operatorname{deg}(\mathcal{K})>0, H^{1}(E, \mathcal{K})=0$, and so the long exact cohomology sequence for the sequence in (8.10) produces the desired surjection (8.7).

Proof of Proposition 8.1. Let .., $\mathcal{L}_{-1}, \mathcal{L}_{0}, \mathcal{L}_{1}, \ldots$ be translates of $\mathcal{L}$ and write

$$
\begin{aligned}
\mathcal{L}^{\prime} & =\cdots \mathcal{L}_{-1} \otimes \mathcal{L}_{0} \\
\mathcal{L}^{\prime \prime} & =\mathcal{L}_{1} \otimes \mathcal{L}_{2} \cdots
\end{aligned}
$$

Now apply Lemma 8.6 repeatedly to conclude that the map

$$
\cdots \otimes H^{0}\left(E^{g}, \mathcal{L}_{-1}\right) \otimes H^{0}\left(E^{g}, \mathcal{L}_{0}\right) \otimes H^{0}\left(E^{g}, \mathcal{L}^{\prime \prime}\right) \longrightarrow H^{0}\left(E^{g}, \mathcal{L}^{\prime} \otimes \mathcal{L}^{\prime \prime}\right)
$$

is onto, and note that the latter map factors through the map in (8.1).

\section{Relations for $B\left(E^{g}, \sigma, \mathcal{L}\right)$}

In this section, $\sigma$ is an arbitrary translation automorphism of $E^{g}$ and we assume that $\mathcal{L}:=\mathcal{L}_{n / k}$ is very ample, or equivalently, that $n_{i} \geq 3$ for all $i$. We will show that the relations for $B\left(E^{g}, \sigma, \mathcal{L}\right)$ are generated in degree $\leq 3$.

\subsection{Notation}

Throughout this section, $\pi: E^{g} \rightarrow E$ denotes the morphism $\pi\left(x_{1}, \ldots, x_{g}\right)=x_{g}$. We will often write $X$ for $E^{g}$ and use the following notation for various $\mathcal{O}_{E^{g}}$-modules:

$$
\begin{aligned}
\mathcal{M}=\mathcal{M}_{m} & =\sigma^{*} \mathcal{L} \otimes \cdots \otimes\left(\sigma^{*}\right)^{m} \mathcal{L}, \\
\mathcal{N}=\mathcal{N}_{m} & =\left(\sigma^{m+1}\right)^{*} \mathcal{L}, \\
\mathcal{K}=\mathcal{K}_{m} & =\operatorname{ker}\left(H^{0}\left(E^{g}, \mathcal{N}_{m}\right) \otimes \mathcal{O}_{X} \rightarrow \mathcal{N}_{m}\right), \\
\mathcal{G} & =\mathcal{G}_{m}=\mathcal{M} \otimes \mathcal{K}=\operatorname{ker}\left(\mathcal{M} \otimes H^{0}\left(E^{g}, \mathcal{N}\right) \rightarrow \mathcal{M} \otimes \mathcal{N}\right) .
\end{aligned}
$$

Since $\mathcal{L}$ is very ample, so is $\mathcal{N}_{m}$ for all $m \geq 0$. Hence both are generated by their global sections. By Lemma 8.3 and Lemma 4.8(4), $\pi_{*} \mathcal{L}$ and $\pi_{*} \mathcal{N}_{m}$ are also generated by their global sections. 
By Lemma 2.11, the relations for $B\left(E^{g}, \sigma, \mathcal{L}\right)$ are generated in degree $\leq 3$ if and only if the map

$$
H^{0}\left(E^{g}, \mathcal{L}\right) \otimes H^{0}\left(E^{g}, \mathcal{G}_{m}\right) \longrightarrow H^{0}\left(E^{g}, \mathcal{L} \otimes \mathcal{G}_{m}\right)
$$

is onto for all $m \geq 2$. As in previous sections, we use $\pi_{*}$ to reduce the question of whether the map in (9.1) is onto to similar questions on $E$.

\subsection{The surjectivity and kernel of the map $Q_{n, k}(E, \tau) \rightarrow B\left(E^{g}, \sigma, \mathcal{L}_{n / k}\right)$ when $X_{n / k}=E^{g}$}

For the rest of this section we fix an integer $m \geq 2$.

The map in (9.1) factors as

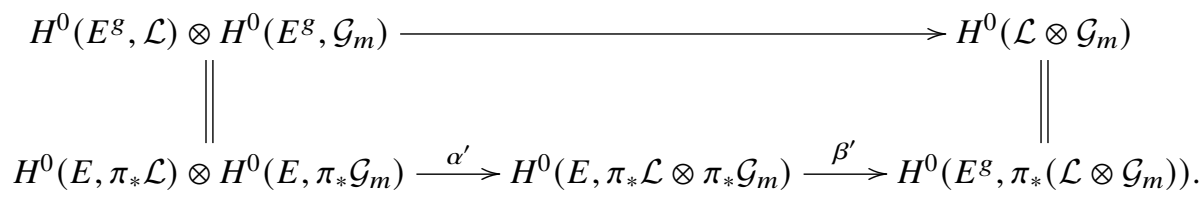

Lemma 9.1. Let

$$
\begin{aligned}
& \alpha: \pi_{*} \mathcal{M} \otimes H^{0}\left(E^{g}, \mathcal{N}\right) \longrightarrow \pi_{*} \mathcal{M} \otimes \pi_{*} \mathcal{N}, \\
& \beta: \pi_{*} \mathcal{M} \otimes \pi_{*} \mathcal{N} \longrightarrow \pi_{*}(\mathcal{M} \otimes \mathcal{N})
\end{aligned}
$$

be the canonical morphisms. There is an exact sequence

$$
0 \longrightarrow \operatorname{ker}(\alpha) \longrightarrow \pi_{*} \mathcal{G} \longrightarrow \operatorname{ker}(\beta) \longrightarrow 0 .
$$

Proof. By its very definition, $\mathcal{G}$ fits into an exact sequence $0 \rightarrow \mathcal{G} \rightarrow \mathcal{M} \otimes H^{0}\left(E^{g}, \mathcal{N}\right) \rightarrow \mathcal{M} \otimes \mathcal{N} \rightarrow 0$. There is an associated exact sequence $0 \rightarrow \pi_{*} \mathcal{G} \rightarrow \pi_{*} \mathcal{M} \otimes H^{0}\left(E^{g}, \mathcal{N}\right) \rightarrow \pi_{*}(\mathcal{M} \otimes \mathcal{N})$ in which the rightmost map factors as

$$
\pi_{*} \mathcal{M} \otimes H^{0}\left(E^{g}, \mathcal{N}\right) \stackrel{\alpha}{\longrightarrow} \pi_{*} \mathcal{M} \otimes \pi_{*} \mathcal{N} \stackrel{\beta}{\longrightarrow} \pi_{*}(\mathcal{M} \otimes \mathcal{N}) .
$$

Thus $\pi_{*} \mathcal{G}=\alpha^{-1}(\operatorname{ker}(\beta))$. Since $\pi_{*} \mathcal{N}$ is generated by its global sections, $H^{0}\left(E, \pi_{*} \mathcal{N}\right) \otimes \mathcal{O}_{E} \rightarrow \pi_{*} \mathcal{N}$ is epic. Hence $\alpha$ is also epic. Its restriction $\alpha^{-1}(\operatorname{ker}(\beta)) \rightarrow \operatorname{ker}(\beta)$ is therefore epic too.

Lemma 9.2. The sequence

$$
0 \longrightarrow \pi_{*} \mathcal{K} \longrightarrow H^{0}\left(E, \pi_{*} \mathcal{N}\right) \otimes \mathcal{O}_{E} \longrightarrow \pi_{*} \mathcal{N} \longrightarrow 0
$$

is exact, and $\pi_{*} \mathcal{K}$ is indecomposable.

Proof. Applying [Har77, Proposition III.9.3] to the projections from $E^{g}=E^{g-1} \times E$ to its two factors, we see that $\pi_{*} \mathcal{O}_{E} g=\mathcal{O}_{E}$. Also, $H^{0}\left(E^{g}, \mathcal{N}\right)=H^{0}\left(E, \pi_{*} \mathcal{N}\right)$.

Since $\mathcal{N}$ is generated by its global sections, the sequence

$$
0 \longrightarrow \mathcal{K} \longrightarrow H^{0}\left(E^{g}, \mathcal{N}\right) \otimes \mathcal{O}_{E^{g}} \longrightarrow \mathcal{N} \longrightarrow 0
$$

is exact. By Lemma 8.3, $\pi_{*} \mathcal{N}$ is indecomposable of slope $>2$ and therefore generated by global sections by Lemma 4.8(4). Thus, applying $\pi_{*}$ to the sequence in (9.4) produces the exact sequence (9.3).

The indecomposability of $\pi_{*} \mathcal{K}$ follows in the same way as Theorem 4.9 or $\$ 4.3 .3$.

Lemma 9.3. Let $\alpha$ and $\beta$ be the maps in Lemma 9.1. Both $\operatorname{ker}(\alpha)$ and $\operatorname{ker}(\beta)$ are semistable locally free $\mathcal{O}_{E}$-modules of slope $>2$. 
Proof. (1) First we deal with $\operatorname{ker}(\alpha)$.

By Lemma 8.6, $\pi_{*} \mathcal{M}$ is semistable. By Lemma 8.5 and Proposition $8.2(2), \mu\left(\pi_{*} \mathcal{M}\right)=m \mu\left(\pi_{*} \mathcal{L}\right)=$ $m \cdot \frac{n}{k^{\prime}}$.

Since $H^{0}\left(E^{g}, \mathcal{N}\right)=H^{0}\left(E, \pi_{*} \mathcal{N}\right)$,

$$
\operatorname{ker}(\alpha)=\pi_{*} \mathcal{M} \otimes \operatorname{ker}\left(H^{0}\left(E^{g}, \mathcal{N}\right) \otimes \mathcal{O}_{E} \rightarrow \pi_{*}(\mathcal{N})\right)=\pi_{*} \mathcal{M} \otimes \pi_{*} \mathcal{K}
$$

by Lemma 9.2. Because the sequence in (9.3) is exact, the degree and rank of $\pi_{*} \mathcal{K}$ are

$$
\begin{aligned}
\operatorname{deg}\left(\pi_{*} \mathcal{K}\right) & =-\operatorname{deg}\left(\pi_{*} \mathcal{N}\right)=-n, \\
\operatorname{rank}\left(\pi_{*} \mathcal{K}\right) & =\operatorname{dim} H^{0}\left(\pi_{*} \mathcal{N}\right)-\operatorname{rank}\left(\pi_{*} \mathcal{N}\right)=n-k^{\prime}
\end{aligned}
$$

by Proposition 8.2(1). Recall that $\frac{n}{k^{\prime}}=\left[n_{g}, \ldots, n_{1}\right]$. Because all $n_{i}$ are $\geq 3, n / k^{\prime}>2$. Hence $n-k^{\prime}>k^{\prime}$, and

$$
\begin{aligned}
\mu(\operatorname{ker} \alpha) & =\mu\left(\pi_{*} \mathcal{M}\right)+\mu\left(\pi_{*} \mathcal{K}\right)=m \cdot \frac{n}{k^{\prime}}-\frac{n}{n-k^{\prime}}=n\left(\frac{m}{k^{\prime}}-\frac{1}{n-k^{\prime}}\right) \\
& >n\left(\frac{m}{k^{\prime}}-\frac{1}{k^{\prime}}\right)=(m-1) \frac{n}{k^{\prime}}
\end{aligned}
$$

which is $>2$.

By Lemma 9.2, $\pi_{*} \mathcal{K}$ is indecomposable, and hence $\operatorname{ker}(\alpha)=\pi_{*} \mathcal{M} \otimes \pi_{*} \mathcal{K}$ is semistable.

(2) Since $\pi_{*} \mathcal{M} \otimes \pi_{*} \mathcal{N}$ and $\pi_{*}(\mathcal{M} \otimes \mathcal{N})$ are semistable of slope $(m+1) \mu\left(\pi_{*} \mathcal{L}\right)$, the kernel of $\beta$ has these properties too.

Lemma 9.4. Let $\mathcal{G}$ be as in $\$ 9.1$. Every indecomposable summand of $\pi_{*} \mathcal{G}$ has slope $>2$.

Proof. By Lemma 9.1, there is an exact sequence $0 \rightarrow \operatorname{ker}(\alpha) \rightarrow \pi_{*} \mathcal{G} \rightarrow \operatorname{ker}(\beta) \rightarrow 0$. Since $\operatorname{ker}(\alpha)$ and $\operatorname{ker}(\beta)$ are semistable of slope $>2$, the result follows from Lemma 4.3.

Lemma 9.5. The multiplication map $\alpha^{\prime}: H^{0}\left(E, \pi_{*} \mathcal{L}\right) \otimes H^{0}\left(E, \pi_{*} \mathcal{G}\right) \rightarrow H^{0}\left(E, \pi_{*} \mathcal{L} \otimes \pi_{*} \mathcal{G}\right)$ is onto.

Proof. We use Corollary 4.11, the assumptions of which can be weakened as follows: all indecomposable summands of $\mathcal{U}$ and $\mathcal{V}$ have slopes $>2$. The result therefore follows from Lemmas 8.3 and 9.4.

Our goal in this section is to show that the map in (9.1) is onto. We will prove this by induction on $g$.

Lemma 9.6. Suppose that the map in (9.1) is onto when $g$ is replaced by $g-1$. The canonical map $\varphi: \pi_{*} \mathcal{L} \otimes \pi_{*} \mathcal{G} \rightarrow \pi_{*}(\mathcal{L} \otimes \mathcal{G})$ is an epimorphism.

Proof. The claim is trivial when $g=1$, so we assume $g \geq 2$.

Let $z \in E$ and let $X_{z}=\pi^{-1}(z) \cong E^{g-1}$. By Grauert's theorem, the fibre of $\varphi$ at $z$ is the map

$$
H^{0}\left(X_{z},\left.\mathcal{L}\right|_{X_{z}}\right) \otimes H^{0}\left(X_{z},\left.\mathcal{G}\right|_{X_{z}}\right) \longrightarrow H^{0}\left(X_{z},\left.\left.\mathcal{L}\right|_{X_{z}} \otimes \mathcal{G}\right|_{X_{z}}\right)
$$

It suffices to show that this map is surjective for all $z$. We will do that.

We will argue by induction on $g$. By replacing $E^{g}$ by $X_{z}, \mathcal{M}$ by $\left.\mathcal{M}\right|_{X_{z}}$ and $\mathcal{N}$ by $\mathcal{M}_{X_{z}}$ in the definitions of $\mathcal{K}$ and $\mathcal{G}$, we obtain sheaves on $X_{z} \cong E^{g-1}$ that we denote by $\mathcal{K}^{\prime}$ and $\mathcal{G}^{\prime}$, respectively. Since $\mathcal{N}$ is locally free, the restriction of the sequence in (9.4) to $X_{z}$ is still exact. Thus we obtain a commutative diagram with exact rows: 


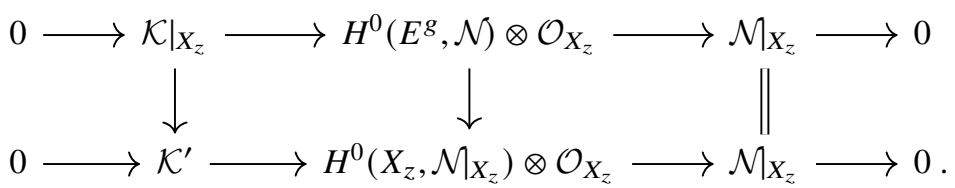

The canonical map $H^{0}\left(E^{g}, \mathcal{N}\right) \rightarrow H^{0}\left(X_{z}, \mathcal{N}_{X_{z}}\right)$ is onto by [CKS19b, Lemma 4.14]. Denote its kernel by $V$. By the Snake Lemma there is an exact sequence

$$
\left.0 \rightarrow V \otimes \mathcal{O}_{X_{z}} \rightarrow \mathcal{K}\right|_{X_{z}} \rightarrow \mathcal{K}^{\prime} \rightarrow 0
$$

Tensoring this with the locally free sheaf $\left.\mathcal{M}\right|_{X_{z}}$ yields the exact sequence

$$
\left.\left.0 \rightarrow V \otimes \mathcal{M}\right|_{X_{z}} \rightarrow \mathcal{G}\right|_{X_{z}} \rightarrow \mathcal{G}^{\prime} \rightarrow 0
$$

Since $\left.\mathcal{L}\right|_{X_{z}}$ is a standard divisor of type $\left(n_{1}, \ldots, n_{g-1}\right)$, it is ample, and so are $\left.\mathcal{M}\right|_{X_{z}}$ and $\left.\left.\mathcal{L}\right|_{X_{z}} \otimes \mathcal{M}\right|_{X_{z}}$. The argument in [CKS19b, Corollary 3.4] shows $H^{1}\left(\left.\mathcal{M}\right|_{X_{z}}\right)=0$ and $H^{1}\left(\left.\left.\mathcal{L}\right|_{X_{z}} \otimes \mathcal{M}\right|_{X_{z}}\right)=0$. Thus we obtain a commutative diagram

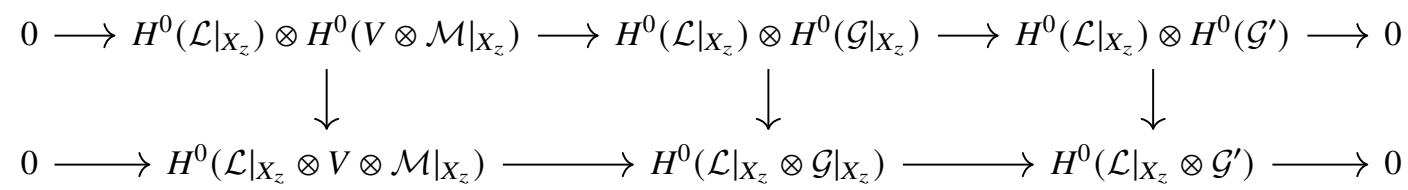

with exact rows. Since the surjectivity of the left (resp., right) vertical map is reduced to the case $X=E^{g-1}$, the hypothesis for $g-1$ (resp., Lemma 8.6) ensures the surjectivity. Therefore the vertical map in the middle is also surjective, and this completes the proof.

We use the following notation in the next proof: if $\mathcal{U}_{1}$ and $\mathcal{U}_{2}$ are $\mathcal{O}_{E}$-modules, we write $\mathcal{K}\left(\mathcal{U}_{1}, \mathcal{U}_{2}\right)$ for the kernel of the canonical morphism $\pi_{*} \mathcal{U}_{1} \otimes \pi_{*} \mathcal{U}_{2} \longrightarrow \pi_{*}\left(\mathcal{U}_{1} \otimes \mathcal{U}_{2}\right)$. If $\mathcal{U}_{1}$ and $\mathcal{U}_{2}$ are locally free $\mathcal{O}_{E^{g}}$-modules, then $\mathcal{K}\left(\mathcal{U}_{1}, \mathcal{U}_{2}\right)$ is obviously a locally free $\mathcal{O}_{E}$-module.

Theorem 9.7. Let $\sigma$ be a translation automorphism of $E^{g}$. If $\mathcal{L}_{n / k}$ is very ample, then the ideal of relations for $B\left(E^{g}, \sigma, \mathcal{L}_{n / k}\right)$ is generated by elements of degree $\leq 3$.

Proof. As explained at the beginning of this section, it suffices to show that the map in (9.1) is onto. We will do that, using induction on $g$ so that we can use the conclusion of Lemma 9.6. First, we will show that $H^{1}(E, \mathcal{K}(\mathcal{L}, \mathcal{G}))=0$.

The exact sequence (9.2) is equal to

$$
0 \rightarrow \pi_{*} \mathcal{M} \otimes \pi_{*} \mathcal{K} \rightarrow \pi_{*} \mathcal{G} \rightarrow \mathcal{K}(\mathcal{M}, \mathcal{N}) \rightarrow 0
$$

Replacing $\mathcal{M}$ by $\mathcal{L} \otimes \mathcal{M}$, the same argument produces the exact sequence

$$
0 \rightarrow \pi_{*}(\mathcal{L} \otimes \mathcal{M}) \otimes \pi_{*} \mathcal{K} \rightarrow \pi_{*}(\mathcal{L} \otimes \mathcal{G}) \rightarrow \mathcal{K}(\mathcal{L} \otimes \mathcal{M}, \mathcal{N}) \rightarrow 0,
$$


since $\mathcal{G}$ is replaced by $\mathcal{L} \otimes \mathcal{G}$. The sequences $\left(\pi_{*} \mathcal{L}\right) \otimes(9.6)$ and (9.7) fit into the commutative diagram

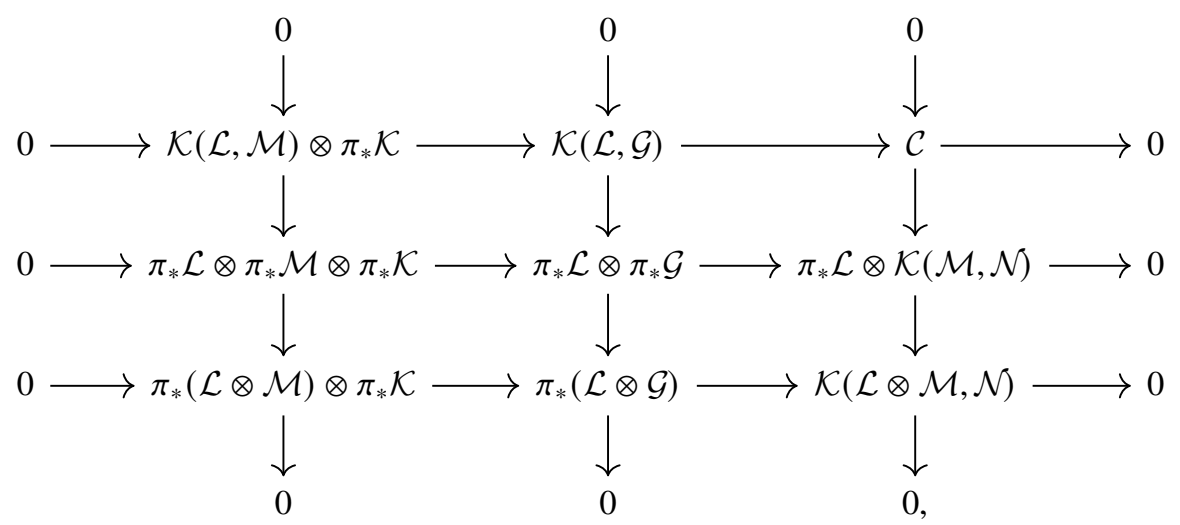

where $\mathcal{C}$ is a locally free $\mathcal{O}_{E}$-module, and the exactness of the vertical sequences follows from Lemma 9.6 and the fact that the canonical morphism $\pi_{*} \mathcal{L} \otimes \pi_{*} \mathcal{M} \rightarrow \pi_{*}(\mathcal{L} \otimes \mathcal{M})$ is an epimorphism. Thus, to show $H^{1}(E, \mathcal{K}(\mathcal{L}, \mathcal{G}))=0$ it suffices to show that $H^{1}\left(E, \mathcal{K}(\mathcal{L}, \mathcal{M}) \otimes \pi_{*} \mathcal{K}\right)=H^{1}(E, \mathcal{C})=0$.

As we showed in Lemma 9.3, $\pi_{*} \mathcal{M} \otimes \pi_{*} \mathcal{K}$ is semistable and has slope $>2$. Thus $\pi_{*} \mathcal{L} \otimes \pi_{*} \mathcal{M} \otimes \pi_{*} \mathcal{K}$ is also semistable and has positive slope. Since $\pi_{*}(\mathcal{L} \otimes \mathcal{M}) \otimes \pi_{*} \mathcal{K}$ has the same property, so does $\mathcal{K}(\mathcal{L}, \mathcal{M}) \otimes \pi_{*} \mathcal{K}$. On the other hand, the exact sequences

$$
0 \rightarrow \pi_{*} \mathcal{L} \otimes \mathcal{K}(\mathcal{M}, \mathcal{N}) \rightarrow \pi_{*} \mathcal{L} \otimes \pi_{*} \mathcal{M} \otimes \pi_{*} \mathcal{N} \rightarrow \pi_{*} \mathcal{L} \otimes \pi_{*}(\mathcal{M} \otimes \mathcal{N}) \rightarrow 0
$$

and

$$
0 \rightarrow \mathcal{K}(\mathcal{L} \otimes \mathcal{M}, \mathcal{N}) \rightarrow \pi_{*}(\mathcal{L} \otimes \mathcal{M}) \otimes \pi_{*} \mathcal{N} \rightarrow \pi_{*}(\mathcal{L} \otimes \mathcal{M} \otimes \mathcal{N}) \rightarrow 0
$$

imply that $\pi_{*} \mathcal{L} \otimes \mathcal{K}(\mathcal{M}, \mathcal{N})$ and $\mathcal{K}(\mathcal{L} \otimes \mathcal{M}, \mathcal{N})$ are semistable and have the same positive slope, whence $\mathcal{C}$ has the same property. It follows from Lemma 4.8 that $H^{1}\left(E, \mathcal{K}(\mathcal{L}, \mathcal{M}) \otimes \pi_{*} \mathcal{K}\right)=H^{1}(E, \mathcal{C})=0$.

Since $0 \rightarrow \mathcal{K}(\mathcal{L}, \mathcal{G}) \rightarrow \pi_{*} \mathcal{L} \otimes \pi_{*} \mathcal{G} \rightarrow \pi_{*}(\mathcal{L} \otimes \mathcal{G}) \rightarrow 0$ is exact and $H^{1}(E, \mathcal{K}(\mathcal{L}, \mathcal{G}))=0$, the map $H^{0}\left(E, \pi_{*} \mathcal{L} \otimes \pi_{*} \mathcal{G}\right) \rightarrow H^{0}\left(E, \pi_{*}(\mathcal{L} \otimes \mathcal{G})\right)=H^{0}(E, \mathcal{L} \otimes \mathcal{G})$ is onto. It follows from Lemma 9.5 that the map in (9.1) is onto. The proof is complete.

Acknowledgements. The first author was partially supported by NSF grants DMS-1565226, DMS-1801011 and DMS-2001128.

The second author was a JSPS Overseas Research Fellow, and supported by JSPS KAKENHI Grant Numbers JP16H06337, JP17K14164 and JP20K14288, Leading Initiative for Excellent Young Researchers, MEXT, Japan, and Osaka City University Advanced Mathematical Institute (MEXT Joint Usage/Research Center on Mathematics and Theoretical Physics JPMXP0619217849). He would like to express his deep gratitude to Paul Smith for his hospitality as a host researcher during the second author's visit to the University of Washington.

The third author thanks Sándor Kovács, Jack Lee, Max Lieblich and Bianca Viray for many helpful conversations.

Conflict of Interest: None.

\section{References}

[AS87] M. Artin and W. F. Schelter, 'Graded algebras of global dimension 3', Adv. Math. 66(2) (1987), 171-216. MR 917738 (88k:16003)

[Ati57] M. F. Atiyah, 'Vector bundles over an elliptic curve', Proc. Lond. Math. Soc. (3) 7 (1957), 414-452. MR 0131423

[ATVdB90] M. Artin, J. Tate and M. Van den Bergh, 'Some algebras associated to automorphisms of elliptic curves', in The Grothendieck Festschrift, Vol. I, Progr. Math., 86 (Birkhäuser Boston, Boston, MA, 1990), 33-85. MR 1086882 (92e:14002)

[ATVdB91] M. Artin, J. Tate and M. Van den Bergh, 'Modules over regular algebras of dimension 3', Invent. Math. 106(2) (1991), 335-388. MR 1128218 (93e:16055) 
[AVdB90] M. Artin and M. Van den Bergh, 'Twisted homogeneous coordinate rings', J. Algebra 133(2) (1990), $249-271$. MR 1067406 (91k:14003)

[AZ94] M. Artin and J. J. Zhang, 'Noncommutative projective schemes', Adv. Math. 109(2) (1994), 228-287. MR 1304753 (96a:14004)

[Bel80] A. A. Belavin, 'Discrete groups and integrability of quantum systems', Funktsional. Anal. i Prilozhen. 14(4) (1980), 18-26, 95. MR 595725

[But94] D. C. Butler, 'Normal generation of vector bundles over a curve', J. Differential Geom. 39(1) (1994), 1-34. MR 1258911

[CC81] D. V. Chudnovsky and G. V. Chudnovsky, 'Completely $X$-symmetric $S$-matrices corresponding to theta functions', Phys. Lett. A 81(2-3) (1981), 105-110. MR 597095

[CC93] F. Catanese and C. Ciliberto, 'Symmetric products of elliptic curves and surfaces of general type with $p_{g}=q=1$ ', J. Algebraic Geom. 2(3) (1993), 389-411. MR 1211993

[Che82] I. V. Cherednik, 'On the properties of factorized S matrices in elliptic functions', Yadernaya Fiz. 36(2) (1982), 549-557. MR 700961

[Che86] I. V. Cherednik, 'On R-matrix quantization of formal loop groups', in Group Theoretical Methods in Physics, Vol. II (Yurmala, 1985) (VNU Scientific Press, Utrecht, 1986), 161-180. MR 919789

[CKS18] A. Chirvasitu, R. Kanda and S. P. Smith, 'Feigin and Odesskii's elliptic algebras', Preprint, 2018 , arXiv:1812. 09550v3.

[CKS19a] A. Chirvasitu, R. Kanda and S. P. Smith, 'Finite quotients of powers of an elliptic curve', Preprint, 2019, arXiv: 1905.06710v3.

[CKS19b] A. Chirvasitu, R. Kanda and S. P. Smith, 'The characteristic variety for Feigin and Odesskii's elliptic algebras', Preprint, 2019, arXiv:1903.11798v4.

[CKS20] A. Chirvasitu, R. Kanda and S. P. Smith, 'Elliptic R-matrices and Feigin and Odesskii’s elliptic algebras', Preprint, 2020, arXiv:2006.12283v1.

[CSW18] A. Chirvasitu, S. P. Smith and L. Z. Wong, 'Noncommutative geometry of homogenized quantum sl(2, C)', Pacific J. Math. 292(2) (2018), 305-354. MR 3733976

[FO89] B. L. Feigin and A. V. Odesskii, 'Sklyanin algebras associated with an elliptic curve', Preprint deposited with Institute of Theoretical Physics of the Academy of Sciences of the Ukrainian SSR, 1989.

[Gus90] N. P. Gushel', 'Very ample divisors on projective bundles over an elliptic curve', Mat. Zametki 47(6) (1990), 15-22, 158. MR 1074523

[Har77] R. Hartshorne, Algebraic Geometry, Grad. Texts in Math., 52 (Springer-Verlag, New York, 1977). MR 0463157 (57 \#3116)

[Hul86] K. Hulek, 'Projective geometry of elliptic curves', Astérisque 137 (1986), 143. MR 845383

[Kee00] D. S. Keeler, 'Criteria for $\sigma$-ampleness', J. Amer. Math. Soc. 13(3) (2000), 517-532. MR 1758752

[Kem91] G. R. Kempf, Complex Abelian Varieties and Theta Functions, Universitext (Springer-Verlag, Berlin, 1991). MR 1109495

[Koi76] S. Koizumi, 'Theta relations and projective normality of Abelian varieties', Amer. J. Math. 98(4) (1976), 865-889. MR 480543

[Lev92] T. Levasseur, 'Some properties of noncommutative regular graded rings', Glasg. Math. J. 34(3) (1992), $277-300$. MR 1181768 (93k:16045)

[LS93] T. Levasseur and S. P. Smith, 'Modules over the 4-dimensional Sklyanin algebra', Bull. Soc. Math. France 121(1) (1993), 35-90. MR 1207244 (94f:16054)

[Mar81] M. Maruyama, 'The theorem of Grauert-Mülich-Spindler', Math. Ann. 255(3) (1981), 317-333. MR 615853

[MFK94] D. Mumford, J. Fogarty and F. Kirwan, Geometric Invariant Theory, 3rd ed., Ergeb. Math. Grenzgeb. (2), 34 (Springer-Verlag, Berlin, 1994). MR 1304906

[Mum70] D. Mumford, 'Varieties defined by quadratic equations', in Questions on Algebraic Varieties (C.I.M.E., III Ciclo, Varenna, 1969) (Edizioni Cremonese, Rome, 1970), 29-100. MR 0282975

[Mum08] D. Mumford, Abelian Varieties, Tata Institute of Fundamental Research Studies in Mathematics, 5 (Hindustan Book Agency, New Delhi, 2008; published for the Tata Institute of Fundamental Research, Bombay, corrected reprint of the 2nd [1974] ed.). With appendices by C. P. Ramanujam and Yuri Manin. MR 2514037 (2010e:14040)

[Mur64] J. P. Murre, 'On contravariant functors from the category of pre-schemes over a field into the category of abelian groups (with an application to the Picard functor)', Publ. Math. Inst. Hautes Études Sci. 23 (1964), 5-43. MR 0206011

[Ode02] A. V. Odesskii, 'Elliptic algebras', Uspekhi Mat. Nauk 57(6[348]) (2002), 87-122. MR 1991863

[OF89] A. V. Odesskii and B. L. Feigin, 'Sklyanin elliptic algebras', Funktsional. Anal. i Prilozhen. 23(3) (1989), 45-54, 96. MR 1026987 (91e:16037)

[Pol03] A. Polishchuk, Abelian Varieties, Theta Functions and the Fourier Transform, Cambridge Tracts in Math., 153 (Cambridge University Press, Cambridge, UK, 2003). MR 1987784

[Pol05] F. Polizzi, 'On surfaces of general type with $p_{g}=q=1, K^{2}=3$ ', Collect. Math. 56(2) (2005), 181-234. MR 2154303 
[PP04] G. Pareschi and M. Popa, 'Regularity on abelian varieties. II. Basic results on linear series and defining equations', J. Algebraic Geom. 13(1) (2004), 167-193. MR 2008719

[Sk182] E. K. Sklyanin, 'Some algebraic structures connected with the Yang-Baxter equation', Funktsional. Anal. $i$ Prilozhen. 16(4) (1982), 27-34, 96. MR 684124 (84c:82004)

[Sk183] E. K. Sklyanin, 'Some algebraic structures connected with the Yang-Baxter equation. Representations of a quantum algebra', Funktsional. Anal. i Prilozhen. 17(4) (1983), 34-48. MR 725414 (85k:82011)

[Smi04] S. P. Smith, 'Maps between non-commutative spaces', Trans. Amer. Math. Soc. 356(7) (2004), 2927-2944. MR 2052602

[Smi16] S. P. Smith, 'Corrigendum to "Maps between non-commutative spaces" [Trans. Amer. Math. Soc., 356(7) (2004) 2927-2944]', Trans. Amer. Math. Soc. 368(7) (2016), 8295-8302 (electronic). MR 2052602 (2005f:14004)

[SS15] S. V. Sam and A. Snowden, 'Stability patterns in representation theory', Forum Math. Sigma 3 (2015), e11, 108. MR 3376738

[SS92] S. P. Smith and J. T. Stafford, 'Regularity of the four-dimensional Sklyanin algebra', Compos. Math. 83(3) (1992), 259-289. MR 1175941 (93h:16037)

[ST01] P. Seidel and R. Thomas, 'Braid group actions on derived categories of coherent sheaves', Duke Math. J. 108(1) (2001), 37-108. MR 1831820

[ST94] S. P. Smith and J. T. Tate, 'The center of the 3-dimensional and 4-dimensional Sklyanin algebras', in Proceedings of Conference on Algebraic Geometry and Ring Theory in honor of Michael Artin, Part I (Antwerp, 1992), K-theory 8(1) (1994), 19-63. MR 1273835

[SVdB13] S. P. Smith and M. Van den Bergh, 'Noncommutative quadric surfaces', J. Noncommut. Geom. 7(3) (2013), 817856. MR 3108697

[Swe69] M. E. Sweedler, Hopf Algebras, Mathematics Lecture Note Series (W. A. Benjamin, Inc., New York, 1969). MR 0252485 (40 \#5705)

[The18] The Stacks Project Authors, 'Stacks Project', 2018, https://stacks.math.columbia.edu.

[Tra85] C. A. Tracy, 'Embedded elliptic curves and the Yang-Baxter equations', Phys. D 16(2) (1985), 203-220. MR 796270

[Tu93] L. W. Tu, 'Semistable bundles over an elliptic curve', Adv. Math. 98(1) (1993), 1-26. MR 1212625

[TVdB96] J. T. Tate and M. Van den Bergh, 'Homological properties of Sklyanin algebras', Invent. Math. 124(1-3) (1996), 619-647. MR 1369430 (98c:16057)

[VdB01] M. Van den Bergh, 'Blowing up of non-commutative smooth surfaces', Mem. Amer. Math. Soc. 154(734) (2001). MR 1846352 (2002k:16057) 